\title{
Geotechnical stability analysis
}

\author{
S. W. SLOAN*
}

\begin{abstract}
This paper describes recent advances in stability analysis that combine the limit theorems of classical plasticity with finite elements to give rigorous upper and lower bounds on the failure load. These methods, known as finite-element limit analysis, do not require assumptions to be made about the mode of failure, and use only simple strength parameters that are familiar to geotechnical engineers. The bounding properties of the solutions are invaluable in practice, and enable accurate limit loads to be obtained through the use of an exact error estimate and automatic adaptive meshing procedures. The methods are very general, and can deal with heterogeneous soil profiles, anisotropic strength characteristics, fissured soils, discontinuities, complicated boundary conditions, and complex loading in both two and three dimensions. A new development, which incorporates pore water pressures in finite-element limit analysis, is also described. Following a brief outline of the new techniques, stability solutions are given for several practical problems, including foundations, anchors, slopes, excavations and tunnels.
\end{abstract}

KEYWORDS: anchors; bearing capacity; excavation; numerical modelling; plasticity; slopes; tunnels

\section{STABILITY ANALYSIS}

In geotechnical engineering, stability analysis is used to predict the maximum load that can be supported by a geostructure without inducing failure. This ultimate load, which is also known as the limit or collapse load, can be used to determine the allowable working load by dividing it by a predetermined factor of safety. The precise value of this factor depends on the type of problem, with, for example, lower values being appropriate for slopes and higher values being adopted for foundations. Rather than impose a factor of safety on the ultimate load to obtain the allowable working load, it is also possible to apply a factor of safety to the strength parameters prior to performing the stability analysis. In some procedures - finite-element strength reduction analysis, for example - the actual safety factor on the strength can be found for a given set of applied loads and material parameters (which typically comprise the cohesion and friction angle).

Once the allowable load is known, the working deformations are usually determined using some form of settlement analysis. Historically, these deformations have been predicted using elasticity theory, but they are now often found from a variety of numerical methods, including non-linear finiteelement analysis. In some cases, particularly those involving dense sands, serviceability constraints on the deformations may actually control the allowable load rather than the ultimate load-carrying capacity.

Broadly speaking, there are four main methods for performing geotechnical stability analysis: limit equilibrium, limit analysis, slip-line methods, and the displacement finiteelement method. In the following, all these techniques will be discussed except the slip-line methods. This family of procedures is omitted, not because they are considered to be ineffective, but simply because they are not well suited to the development of general-purpose software which can deal with a wide variety of practical problems.

Manuscript received 14 September 2012; revised manuscript accepted 22 January 2013.

* ARC Centre of Excellence for Geotechnical Science and Engineering, University of Newcastle, NSW, Australia.

\section{Limit equilibrium}

Limit equilibrium is the oldest method for performing stability analysis, and was first applied in a geotechnical setting by Coulomb (1773). In its most basic form, this approach presupposes a failure mechanism, and implicitly assumes that the stresses on the failure planes are limited by the traditional strength parameters $c$ and $\phi$. The chief advantages of the limit-equilibrium method are its simplicity and its long history of use, which have resulted in widely available software and extensive collective experience concerning its reliability. Its main disadvantage, on the other hand, is the need to guess the general form of the failure surface in advance, with poor choices giving poor estimates of the failure load. In practice, the correct form of the failure surface is often not intuitively obvious, especially for problems with an irregular geometry, complex loading, or complicated stratigraphy. There are other shortcomings of the technique, as follows.

(a) The resulting stresses do not satisfy equilibrium at every point in the domain.

(b) There is no simple means of checking the accuracy of the solution.

(c) It is hard to incorporate anisotropy and inhomogeneity.

(d) It is difficult to generalise the procedure from two to three dimensions.

Despite these limitations, a multitude of limit-equilibrium methods have been proposed and implemented, particularly for slope stability analysis. Indeed, early examples of widely used slope stability methods include those of Janbu (1954, 1973), Bishop (1955), Morgenstern \& Price (1965), Spencer (1967) and Sarma (1973, 1979). A more recent procedure, described by Donald \& Giam (1989b), is also noteworthy, since it gives a factor of safety that is a strict upper bound on the true value.

The key principles of the limit-equilibrium approach can be illustrated by considering the classical bearing capacity problem for a smooth strip footing, of width $B$, resting on a deep layer of undrained clay of strength $s_{\mathrm{u}}$, as shown in Fig. 1 .

To begin the analysis, the supposition is made that failure occurs along a circular surface whose centre lies at some point directly above the edge of the footing, as shown in Fig. 2. In addition, undrained failure along this surface is 


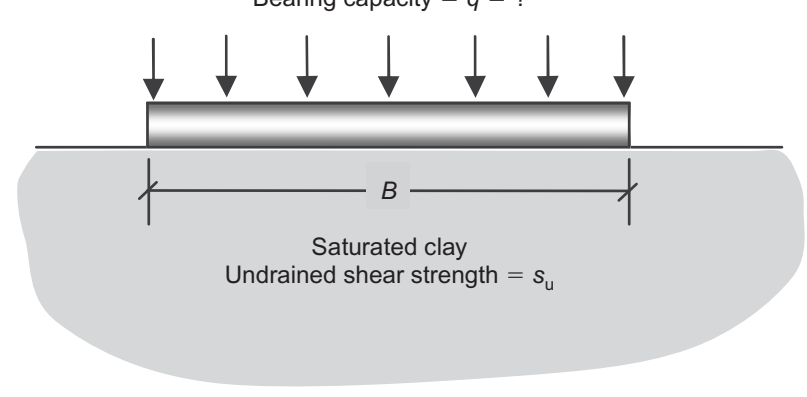

Fig. 1. Smooth strip footing on deep layer of undrained clay

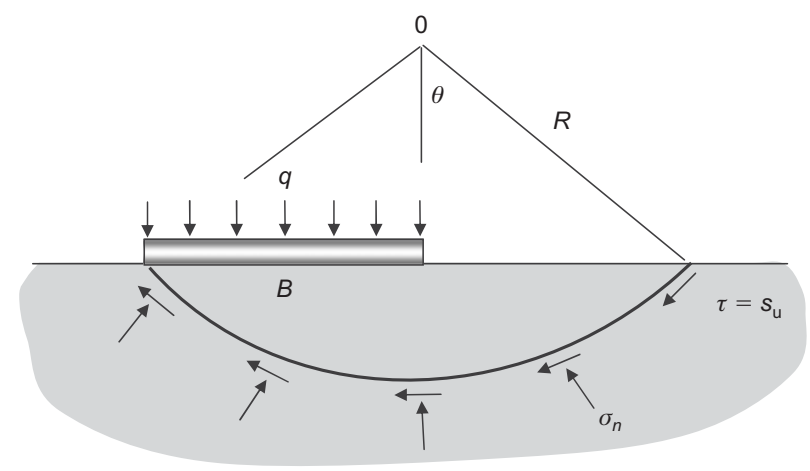

Fig. 2. Limit-equilibrium failure surface for strip footing on clay

assumed to be governed by the Tresca criterion, with the maximum shear strength being fully mobilised at every point, so that the shear stress is given by $\tau=s_{\mathrm{u}}$.

Taking moments about the centre of the failure surface, $\mathrm{O}$, the following is obtained.

$$
(q B) \times \frac{B}{2}=\left(2 R \theta \times s_{\mathrm{u}}\right) \times R
$$

or

$$
q=\frac{4 s_{\mathrm{u}} \theta}{\sin ^{2} \theta}
$$

The lowest value of $q$, and hence the geometry of the critical surface, can be found by setting $\mathrm{d} q / \mathrm{d} \theta=0$. This leads to the simple non-linear equation $\tan \theta-2 \theta=0$, which can be solved to yield the critical angle $\theta_{\mathrm{c}}=66.8^{\circ}$. Inserting this critical angle in equation (1) gives the approximate bearing capacity as

$$
q=5 \cdot 52 s_{\mathrm{u}}
$$

which is approximately $7 \%$ above the exact solution $q=(2+\pi) s_{\mathrm{u}}$ derived by Prandtl (1920).

\section{Limit analysis}

Limit analysis is based on the plastic bounding theorems developed by Drucker et al. (1951, 1952), and assumes small deformations, a perfectly plastic material (Fig. 3(a)), and an associated flow rule (Fig. 3(b)). The last assumption, which is often termed the normality rule, implies that the plastic strain rates $\dot{\varepsilon}_{i j}^{\mathrm{p}}$ are normal to the yield surface, $f\left(\sigma_{i j}\right)$, so that $\dot{\varepsilon}_{i j}^{\mathrm{p}}=\dot{\lambda} \partial f / \partial \sigma_{i j}$, where $\dot{\lambda}$ is a non-negative plastic multiplier. For this type of plasticity model it is necessary to work with velocities and strain rates, rather than displacements and strains, as the latter become undefined at collapse.

The lower-bound theorem is based on the principle of a statically admissible stress field. Such a stress field is defined as one that satisfies equilibrium, the stress boundary condi-

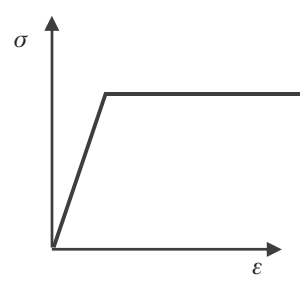

(a)

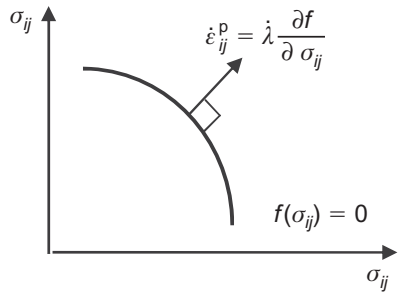

(b)
Fig. 3. (a) Perfectly plastic material model and (b) associated flow rule

tions and the yield criterion. For a perfectly plastic material model with an associated flow rule, it can be shown that the load supported by a statically admissible stress field is a lower bound on the true limit load. Although the limit load for such a material is unique, the optimum stress field is not, and thus it is possible to have a variety of stress fields that furnish the same lower bounds. To illustrate the application of lower-bound limit analysis, the smooth rigid footing problem shown in Fig. 1 is considered again. The simple stress field shown in Fig. 4, which consists of three distinct zones separated by two vertical stress discontinuities, is statically admissible since it satisfies equilibrium, the stress boundary conditions, and the undrained (Tresca) yield criterion $\sigma_{1}-\sigma_{3}=2 s_{\mathrm{u}}$ everywhere in the domain. Note that each stress discontinuity is statically admissible because the normal and shear stresses are the same on both of its opposing sides, and that equilibrium is automatically satisfied everywhere in each zone because the unit weight is zero and the stress field is constant. Although the normal and shear stresses must be continuous across an admissible stress discontinuity, the normal stress on a plane orthogonal to the discontinuity is permitted to jump. This feature can be exploited in the construction of stress fields to give useful lower bounds, and is shown in Fig. 4.

Since the stress field supports a vertical principal stress of $\sigma_{1}=4 s_{\mathrm{u}}$ in the zone beneath the footing, this defines a lower bound on the bearing capacity of $q_{\text {low }}=4 s_{\mathrm{u}}$.

In contrast to the lower-bound theorem, the upper-bound theorem requires the computation of a kinematically admissible velocity field that satisfies the velocity boundary conditions and the plastic flow rule. For such a field, an upper bound on the collapse load is obtained by equating the power expended by the external loads to the power dissipated internally by plastic deformation. Note that although the true limit load from such a calculation is unique, the actual failure mechanism is not. This implies that multiple mechanisms may give the same limit load, and it is necessary to seek the mechanism that gives the lowest upper bound. A simple upper-bound mechanism for this strip footing example, shown in Fig. 5, assumes that failure occurs by the rigid-body rotation of a circular segment, with

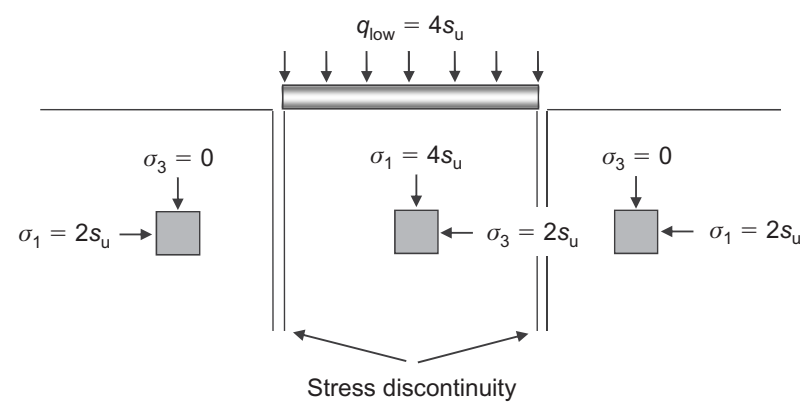

Fig. 4. Lower-bound stress field for strip footing on clay 


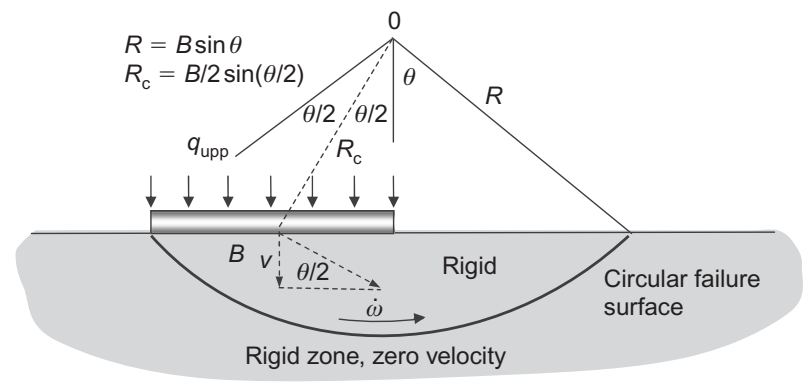

Fig. 5. Upper-bound failure mechanism for strip footing on clay

all the internal energy being dissipated along the velocity discontinuity. From the geometry of Fig. 5, the rate of internal energy (i.e. power) dissipation is

$$
\begin{aligned}
\dot{W}_{\text {int }} & =P_{\text {int }}=\int \Delta u_{\mathrm{s}} s_{\mathrm{u}} \mathrm{d} L \\
& =(R \dot{\omega}) \times s_{\mathrm{u}} \times(2 R \theta)
\end{aligned}
$$

where $\dot{\omega}$ is the angular velocity of the segment about point 0 , and $\Delta u_{\mathrm{s}}$ is the tangential velocity jump across the discontinuity. Equating this quantity to the rate of work (i.e. power) expended by the external forces

$$
\begin{aligned}
\dot{W}_{\mathrm{ext}} & =P_{\mathrm{ext}}=q_{\mathrm{upp}} B \times v \\
& =q_{\mathrm{upp}} B \times R_{\mathrm{c}} \dot{\omega} \sin \left(\frac{\theta}{2}\right) \\
& =q_{\mathrm{upp}} B \times \frac{B}{2} \dot{\omega}
\end{aligned}
$$

and substituting for $R$ gives

$$
q_{\text {upp }}=\frac{4 s_{\mathrm{u}} \theta}{\sin ^{2} \theta}
$$

Setting $\mathrm{d} q_{\text {upp }} / \mathrm{d} \theta=0$ furnishes the critical angle $\theta_{\mathrm{c}}=66 \cdot 8^{\circ}$, which in turn gives the lowest (optimal) upper bound for this mechanism as

$$
q_{\text {upp }}=5 \cdot 52 s_{\mathrm{u}}
$$

Combining this result with the previous lower-bound estimate, the exact bearing capacity for the footing on the ideal material in this analysis must lie within the range

$$
4 s_{\mathrm{u}} \leqslant q \leqslant 5 \cdot 52 s_{\mathrm{u}}
$$

Although the limit-equilibrium and upper-bound calculations give the same estimate of the bearing capacity for this case, their results are generally different for more complex failure mechanisms where the limit-equilibrium solution may not be kinematically admissible. Notwithstanding the limitations that stem from the assumption of a simple perfectly plastic material model, the ability of the limit theorems to provide rigorous bounds on the collapse load is one of their great attractions. Indeed, for complex practical problems where the failure load is difficult to estimate by other methods, this is a compelling advantage, and one of the few instances in non-linear mechanics where the error in an approximate solution can be bounded exactly.

Although the limit theorems can be applied in an analytical setting to give useful bounds for simple problems, discrete numerical formulations provide a more general means of harnessing their power. In particular, finite-element limit analysis formulations have evolved rapidly in recent years, and are now sufficiently developed for large-scale practical applications in geotechnical engineering. These procedures will be the focus of attention in this paper, and inevitably lead to some form of optimisation problem, the solution of which defines either a statically admissible stress field or a kinematically admissible velocity field. Finiteelement formulations of the limit theorems inherit all the advantages of the finite-element method, and can model complex geometries, layered soils, anisotropy, soil-structure interaction, interface effects, discontinuities, complicated loadings, and a wide variety of boundary conditions. The success of this approach, however, hinges on the development of formulations and solution algorithms that are robust, efficient and extendable to three dimensions. Moreover, some means of refining the mesh is needed to ensure that the 'gap' between the upper- and lower-bound limit loads is sufficiently small.

\section{Displacement finite-element analysis}

As a result of the rapid evolution of powerful user-friendly software, displacement finite-element analysis is now widely used in geotechnical practice - not only for the prediction of deformations, but also for the prediction of stability. This method is very general, and can accommodate advanced constitutive models that incorporate non-associated flow, heterogeneity, anisotropy, and work/strain-hardening and softening. In addition, robust procedures are available for modelling interface behaviour, soil-structure interaction and large deformations, as well as fully coupled consolidation and dynamics. When it is used to predict stability under static loading, displacement finite-element analysis can be used in two different modes.

(a) The loads are applied in increments until the deformation response indicates that a state of collapse has been reached. The approximate ultimate load so obtained furnishes a safety factor in terms of force, not strength, and requires a complete simulation of the loaddeformation response (e.g. Sloan, 1979, 1981; Toh \& Sloan, 1980; Sloan \& Randolph, 1982; De Borst \& Vermeer, 1984). Unless advanced procedures (such as arc-length methods) are used, this approach leads to instability in the calculations at collapse if the problem is loaded by prescribed forces rather than by prescribed displacements.

(b) Successive analyses with reduced strengths are conducted until equilibrium can no longer be maintained (e.g. Zienkiewicz et al., 1975; Dawson et al., 1999; Griffiths \& Lane, 1999). This approach, known as strength reduction analysis, involves monitoring deformations at specified control points in the soil, and gives the safety factor in terms of strength - much like the safety factor that is computed in traditional slope stability calculations using limit equilibrium. Since the method relies on nonconvergence of the finite-element simulations to indicate failure, considerable care must be exercised to ensure that the non-convergence is caused by genuine collapse, and not some other numerical effect.

Figure 6 shows the load-deformation response for a smooth, rigid strip footing on clay, computed using the displacement finite-element program SNAC (Abbo \& Sloan, 2000), for a soil with a rigidity index $G / s_{\mathrm{u}}=100$ and undrained Poisson's ratio $v_{\mathrm{u}}=0.49$. In this example the rigid foundation is simulated by the application of uniform vertical displacements to nodes underneath the footing, and 15-noded (quartic) triangles are used to ensure that the soil deformations are modelled accurately under incompressible conditions (Sloan, 1979, 1981; Sloan \& Randolph, 1982). The finiteelement program SNAC, developed at The University of Newcastle over the past two decades, employs adaptive 


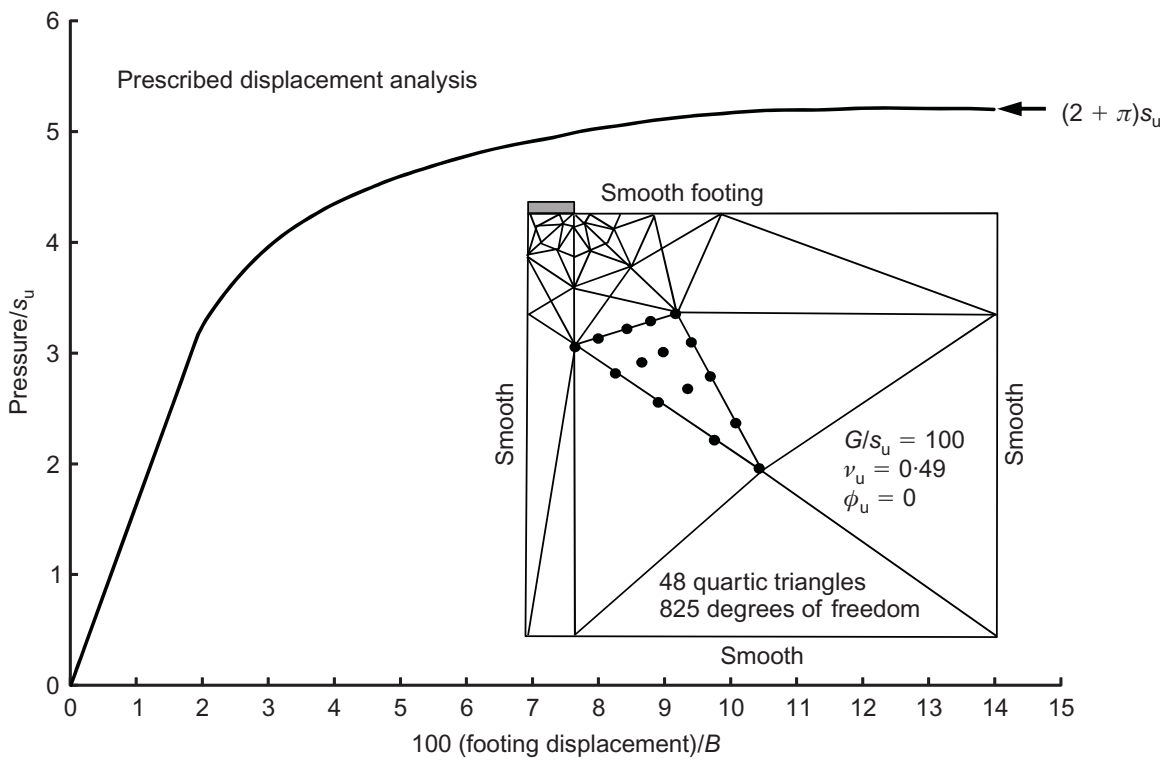

Fig. 6. Displacement finite-element analysis of strip footing on clay

explicit methods to integrate the stress-strain relations and load-deformation response to within a specified accuracy, and is thus well suited to collapse predictions (Sloan, 1987; Abbo \& Sloan, 1996; Sheng \& Sloan, 2001; Sloan et al., 2001). For the mesh shown, the displacement finite-element analysis indicates a clear collapse pressure of $5 \cdot 19 s_{\mathrm{u}}$, which is within $1 \%$ of Prandtl's exact result of $(2+\pi) s_{\mathrm{u}}$. Unlike the methods discussed previously, stability analysis with the displacement finite-element method requires not only the conventional strength parameters, but also the deformation parameters (Poisson's ratio and shear modulus in this case).

Displacement finite-element analysis computes the form of the failure mechanism automatically, and can model a variety of complicated loadings and boundary conditions. The method is not for the naïve user, however, and even with the advent of sophisticated geotechnical software considerable care and experience are required to use the procedure with confidence in geotechnical practice (Potts, 2003). Since a displacement finite-element solution satisfies equilibrium and the flow rule only in a 'weak' sense over the domain, the quality of the resulting collapse load prediction is often critically dependent on the mesh adopted. Sensitivity studies, using successively finer meshes, are generally advisable to confirm the accuracy of the computed limit load, since no reliable error estimate is available for the elasto-plastic models commonly used in geotechnical analysis. In addition, the accuracy of the limit load can be affected by the number of load steps used in the analysis (Sloan, 1981; Abbo \& Sloan, 1996; Sheng \& Sloan, 2001), the numerical integration scheme used to evaluate the elasto-plastic stresses (Potts \& Gens, 1985; Sloan, 1987; Sloan et al., 2001), the tolerances used to check convergence of the global equilibrium iterations, and the type of element employed (Nagtegaal et al., 1974; Sloan, 1979, 1981; Toh \& Sloan, 1980; Sloan \& Randolph, 1982). Of these factors, the correct choice of element is particularly crucial for stability analysis, since the incompressibility constraint imposed by undrained analysis may lead to 'locking' where the load-deformation response rises continuously with increasing deformation, regardless of the mesh discretisation adopted. This phenomenon is due to constraints on the nodal displacements, generated by the incompressibility condition, multiplying at a faster rate than the degrees of freedom as the mesh is refined, and it is especially pronounced for axisymmetric loading with loworder elements (such as the linear three-noded triangle and four-noded quadrilateral). Locking can also occur for displacement finite-element analysis with the Mohr-Coulomb model, which involves dilatational plastic shearing and is widely used for drained stability predictions (Sloan, 1981). To ensure that an element is suitable for accurate collapse load predictions, under both undrained and drained conditions, three different strategies have been proposed.

(a) The use of 'reduced' integration in forming the element stiffness matrices (e.g. Zienkiewicz et al., 1975; Zienkiewicz, 1979; Griffiths, 1982). This approach, which has been widely used with the quadratic eight-noded quadrilateral, reduces the number of constraints on the nodal degrees of freedom at collapse, and introduces additional 'flexibility' into the displacement field by approximate numerical integration of the element stiffness matrices. In general, the method gives good estimates of the collapse load, but may generate unrealistic deformation patterns for some problems (Sloan, 1983; Sloan \& Randolph, 1983). Selective integration methods, which underintegrate the volumetric stiffness terms while fully integrating the deviatoric stiffness terms, may also be used in some cases to alleviate the problem of locking for low-order elements (Malkus \& Hughes, 1978).

(b) The use of high-order triangular elements, with full integration of the stiffness matrices. This approach, first advocated by the author (Sloan, 1979, 1981; Sloan \& Randolph, 1982), follows from the observation that, as meshes of high-order triangles are refined, the new degrees of freedom are added at a faster rate than the nodal constraints imposed by the incompressibility condition, thus avoiding the problem of locking. Since these elements use full integration, no difficulties are encountered with spurious deformation patterns. Although a variety of triangular elements can be shown to be suitable for geotechnical stability analysis, the 15noded triangle, with a quartic displacement expansion, gives good collapse load predictions under both planestrain and axisymmetric loading. This element is also highly effective for drained stability applications involving dilatational plasticity models, and can be implemented to give efficient run times (Sloan, 1979, 1981; Sloan \& Randolph, 1982). For plane-strain deformation, which generates fewer constraints than axisymmetric deformation, the six-noded quadratic triangle with full 
integration is a viable alternative to the 15 -noded triangle, and gives reliable estimates of the collapse load.

(c) The use of mixed pressure-displacement formulations. To avoid numerical oscillations in the solutions, these elements traditionally use a pressure expansion that is one order lower than the displacement expansion (e.g. a sixnoded quadratic displacement triangle with a linear pressure variation interpolated at the corner nodes), but they can also be used in a 'stabilised' form where the pressure and displacement expansions are of equal order (Pastor et al., 1997, 1999). Although they appear to give good results, these formulations are more complicated than the previous two options, and have not been widely adopted for geotechnical stability analysis.

In geotechnical applications, undrained and drained stability analyses can be performed as limiting cases of fully coupled Biot consolidation, with the former case corresponding to a very fast loading rate and the latter case corresponding to a very slow loading rate. Interestingly, when using this approach for stability calculations with a Mohr-Coulomb yield criterion, a non-associated flow rule with a zero (or small) dilation angle should be used to obtain realistic estimates of the collapse load (Small et al., 1976; Small, 1977; Sloan \& Abbo, 1999). If a finite dilation angle is adopted, the loaddeformation response will display a hardening characteristic and fail to asymptote towards a clear collapse state.

\section{Comparison of methods for stability analysis}

Table 1 summarises the key features of the limit equilibrium, limit analysis and displacement finite-element approaches for assessing geotechnical stability. Clearly, the limit-equilibrium method has shortcomings, some of which will be explored further in a later section of this paper, while the displacement finite-element method is the most general. Conventional limit analysis has the intrinsic advantage of providing solutions that bound the collapse load from above and below, but it is restricted to the use of simple soil models and is often difficult to apply in practice. The results in Table 1 suggest that finite-element limit analysis, which combines the generality of the finite-element approach with the rigour of limit analysis, is an appealing alternative to traditional stability prediction techniques. The potential of this type of method will be explored fully in this paper, with a particular focus on its practical utility and scope for future development.

\section{FINITE-ELEMENT LIMIT ANALYSIS}

The theory of finite-element limit analysis is quite different from that of displacement-based finite-element analysis, even though both methods are rooted in the concept of a discrete formulation. Before discussing the fundamental details of finite-element limit analysis, a brief historical review of its development will be given. This review serves to highlight some of the advantages and drawbacks of the approach, as well as its application to practical examples.

Historical development of finite-element lower-bound analysis Lysmer (1970) was an early pioneer in applying finite elements and optimisation theory to compute rigorous lower bounds for plane-strain geotechnical problems. Lysmer's formulation was based on a linear three-noded triangle, with the unknowns being the normal stresses at the end of each side, plus another 'internal' normal stress, and he employed linear programming to solve the resulting optimisation problem. To satisfy the Mohr-Coulomb yield function in its native form, the Cartesian stresses at every point in an element must satisfy a non-linear (quadratic) inequality constraint. To avoid this type of constraint, and thus generate a linear programming problem, Lysmer (1970) linearised the yield surface using an internal polyhedral approximation that replaced each non-linear yield inequality constraint by a series of linear inequalities. The accuracy of the resulting linearisation can be controlled by varying the number of sides in the polyhedral approximation, with the highest accuracy being obtained at the cost of additional constraints and increased solution times. Because the stress field inside each element is assumed to vary linearly, it is sufficient to impose these inequalities at each node to ensure that the linearised yield condition is satisfied throughout the domain, thereby satisfying a key condition of the lower-bound theorem. In addition to the triangular elements used for modelling the soil, Lysmer's formulation also included statically admissible stress discontinuities along the edges between adjacent elements. These greatly enhance the accuracy of a finite-element lower-bound formulation, especially when singularities are present in the stress field (such as at the edge of a rigid footing), and feature prominently in most subsequent implementations of the method. Application of the element equilibrium equations, the discontinuity equilibrium equations and the stress boundary conditions leads to a set of equality constraints on the unknown stresses, while, as described above, the linearised yield criterion generates a large set of linear inequality constraints. The objective function, which corresponds to the collapse load, is a linear function of the stresses. After assembling all the element and nodal contributions for the mesh, the collapse load, denoted by the quantity $\boldsymbol{c}^{\mathrm{T}} \boldsymbol{\sigma}$, is maximised by solving a linear programming problem of the form

$\begin{array}{lll}\text { Maximise } & \boldsymbol{c}^{\mathrm{T}} \boldsymbol{\sigma} \\ \text { subject to } & \mathbf{A}_{1} \boldsymbol{\sigma}=\boldsymbol{b}_{1} & \begin{array}{l}\text { collapse load } \\ \text { continuum and discontinuity } \\ \text { equilibrium, stress boundary } \\ \text { conditions } \\ \text { linearised yield conditions }\end{array} \\ \qquad \mathbf{A}_{2} \boldsymbol{\sigma} \leqslant \boldsymbol{b}_{2} & \end{array}$

Table 1. Properties of traditional methods used for geotechnical stability analysis

\begin{tabular}{|c|c|c|c|c|}
\hline Property & $\begin{array}{l}\text { Limit } \\
\text { equilibrium }\end{array}$ & $\begin{array}{l}\text { Upper-bound limit } \\
\text { analysis }\end{array}$ & $\begin{array}{l}\text { Lower-bound limit } \\
\text { analysis }\end{array}$ & $\begin{array}{l}\text { Displacement finite-element } \\
\text { analysis }\end{array}$ \\
\hline Assumed failure mechanism? & Yes & Yes & - & No \\
\hline Equilibrium satisfied everywhere? & No (globally) & - & Yes & No (nodes only) \\
\hline Flow rule satisfied everywhere? & No & Yes & - & No (integration points only) \\
\hline $\begin{array}{l}\text { Complex loading and boundary conditions } \\
\text { possible? }\end{array}$ & No & Yes & Yes & Yes \\
\hline Complex soil models possible? & No & No & No & Yes \\
\hline Coupled analysis possible? & No & No & No & Yes \\
\hline Error estimate? & No & Yes (with lower bound) & Yes (with upper bound) & No \\
\hline
\end{tabular}


where $\boldsymbol{c}, \boldsymbol{b}_{1}$ and $\boldsymbol{b}_{2}$ are vectors of constants; $\mathbf{A}_{1}$ and $\mathbf{A}_{2}$ are matrices of constants; and $\boldsymbol{\sigma}$ is a global vector of unknown normal and 'internal' stresses acting on the element edges.

Although Lysmer's finite-element approach for computing lower bounds was a pivotal conceptual advance, it has three significant limitations that prevented it from being used widely in practice. The first of these stems from the choice of variables used in the formulation, which leads to a poorly conditioned system of constraint equations that is highly sensitive to the shape of the elements in the mesh. The second shortcoming of the method is its computational inefficiency, which follows from the use of the simplex algorithm to solve the linear programming problem defined by equation (2). Since the iterations required by this algorithm grow rapidly with the size of the optimisation problem being tackled, the number of elements that can be used in a mesh is severely restricted. The third limitation of the formulation is that it does not include a strategy for "extending' the stress field over a semi-infinite domain so that the equilibrium, stress boundary and yield conditions are satisfied everywhere. This process, also known as 'completing' the stress field, is necessary for the solution to be classed as a rigorous lower bound.

Following Lysmer's seminal work, Anderheggen \& Knöpfel (1972), Pastor (1978) and Bottero et al. (1980) proposed various discrete methods for two-dimensional lower-bound limit analysis that were all based on linear triangles and linear programming. These procedures introduced a number of key improvements, including the use of Cartesian stresses as problem variables to simplify the formulation, and the development of special extension elements for generating complete solutions in semi-infinite media. Soon after, Pastor \& Turgeman (1982) proposed a lower-bound technique for modelling the important case of axisymmetric loading. Although potentially powerful, these early methods were limited by the computational performance of the linear programming codes at the time, and could solve only relatively small problems. Indeed, the practical utility of discrete limit analysis techniques has been strongly linked to the development of efficient algorithms for solving the associated optimisation problems. These problems have special features, including extremely sparse and unsymmetric constraint equations, which must be exploited fully in order to solve large cases efficiently. In an effort to address this issue, Sloan $(1988 \mathrm{a}, 1988 \mathrm{~b})$ proposed a fast linear programming formulation that can solve small- to medium-scale two-dimensional problems on a standard desktop machine. This procedure is based on a novel active set algorithm, which employs a steepest-edge search in the optimisation iterations, and fully exploits the highly sparse nature of the lower-bound constraint matrix. The method has been used successfully to predict the stability of a wide variety of twodimensional problems, including tunnels (Assadi \& Sloan, 1991; Sloan \& Assadi, 1991, 1992), slopes (Yu et al., 1998), foundations (Ukritchon et al., 1998; Merifield et al., 1999), anchors (Merifield et al., 2001, 2006a), braced excavations (Ukritchon et al., 2003), and longwall mine workings (Sloan \& Assadi, 1994).

Although lower-bound methods based on linear programming are capable of providing useful solutions for twodimensional problems of moderate size, they are poorly suited to three-dimensional analysis, as huge numbers of inequalities arise when the yield criterion is linearised. Moreover, it is not always clear how to linearise a threedimensional yield surface in an optimal manner. Both of these issues can be avoided by leaving the yield constraints in their native form and adopting non-linear programming algorithms to solve the resulting optimisation problem. Indeed, with this approach, three-dimensional formulations present no special difficulties, other than adding geometrical complexity and increasing the number of unknowns. An early discrete lower-bound formulation based on non-linear programming was described in Belytschko \& Hodge (1970). This procedure used piecewise-quadratic equilibrium stress fields, and maximised the collapse load, subject to the nonlinear yield constraints, by means of a sequential unconstrained minimisation technique. Although it furnishes rigorous lower bounds, the method proved to be slow for largescale problems. In a subsequent modification of Lysmer's formulation, Basudhar et al. (1979) incorporated the nonlinear yield constraints directly, converted the constrained optimisation problem to an unconstrained one using the extended penalty method of Kavlie \& Moe (1971), and computed the optimal solution (best lower bound) using a variant of the sequential unconstrained minimisation technique (Powell, 1964). Following this work, Arai \& Tagyo (1985) used constant-stress elements, and the sequential unconstrained minimisation technique with the conjugate gradient algorithm of Fletcher \& Reeves (1964), to obtain a statically admissible stress field for geotechnical problems. Although both these non-linear formulations require only a modest number of inequality constraints to ensure that the stresses satisfy the yield criterion, they still proved unsuitable for large-scale geotechnical problems, owing to the computational inefficiency of the methods employed to solve the corresponding optimisation problem.

Lyamin (1999) and Lyamin \& Sloan (2002a) dramatically improved the practical utility of the discrete lower-bound method by employing linear stress elements, imposing the non-linear yield conditions in their native form, and solving the resulting non-linear optimisation problem using a variant of an algorithm developed for mixed limit analysis formulations (Zouain et al., 1993). After assembling all the element and nodal contributions, the load carried by the unknown stresses and body forces, denoted by $\boldsymbol{c}_{1}^{\mathrm{T}} \boldsymbol{\sigma}$ and $\boldsymbol{c}_{2}^{\mathrm{T}} \boldsymbol{h}$ respectively, is maximised by solving the following non-linear programming problem

$$
\begin{aligned}
& \begin{array}{l}
\text { Maximise } \\
\boldsymbol{c}_{1}^{\mathrm{T}} \boldsymbol{\sigma}+\boldsymbol{c}_{2}^{\mathrm{T}} \boldsymbol{h}
\end{array} \\
& \begin{array}{lll}
\text { subject to } & \text { collapse load or body force } \\
\mathbf{A}_{11} \boldsymbol{\sigma}+\mathbf{A}_{12} \boldsymbol{h} & =\boldsymbol{b}_{1} & \text { continuum equilibrium } \\
\mathbf{A}_{2} \boldsymbol{\sigma} & \boldsymbol{b}_{2} & \text { discontinuity equilibrium, } \\
& & \text { stress boundary conditions } \\
& f\left(\boldsymbol{\sigma}^{i}\right) \leqslant \boldsymbol{0} & \text { yield conditions for each node } i
\end{array}
\end{aligned}
$$

where $\boldsymbol{c}_{1}, \boldsymbol{c}_{2}, \boldsymbol{b}_{1}$ and $\boldsymbol{b}_{2}$ are vectors of constants; $\mathbf{A}_{11}, \mathbf{A}_{12}$ and $\mathbf{A}_{2}$ are matrices of constants; $f$ is the non-linear yield criterion; $\boldsymbol{\sigma}^{i}$ is a local vector of Cartesian stresses at node $i$; $\boldsymbol{\sigma}$ is a global vector of unknown Cartesian stresses; and $\boldsymbol{h}$ is a global vector of unknown body forces acting on each element. Including the body forces in the formulation permits stability numbers based on the unit weight to be optimised, and is especially useful in predicting the load capacity of slopes, tunnels and excavations. The solution method used by Lyamin \& Sloan (2002a) is an interior point, two-stage, quasi-Newton scheme that exploits the underlying structure of the lower-bound optimisation problem. Since its iteration count is largely independent of the grid refinement for a given problem, the method is able to handle large-scale two-dimensional meshes with several thousand elements in a few seconds, and is many times faster than traditional linear programming formulations. The detailed timing comparisons presented by Lyamin \& Sloan 
(2002a) suggest that, compared with the linear programming approach of Sloan (1988a), their technique typically gives at least a 50-fold reduction in CPU time for large two-dimensional problems. Further advantages include the ability to model three-dimensional problems, where the number of unknowns can be huge, as well as any type of convex yield criterion. Thanks to its efficiency and robustness, the lowerbound method of Lyamin \& Sloan (2002a) has been used to predict the stability of a wide range of geotechnical problems, including tunnels (Lyamin \& Sloan, 2000), sinkholes and cavities (Augarde et al., 2003a, 2003b), two- and threedimensional foundations on clay and/or sand (Shiau et al., 2003; Hjiaj et al., 2004, 2005; Salgado et al., 2004), anchors in clay or sand (Merifield et al., 2003, 2005, 2006a), foundations on rock (Merifield et al., 2006b), and slopes in soil or rock (Li et al., 2008, 2009a, 2009b, 2010). Following the work of Lyamin \& Sloan (2002a), Krabbenhøft \& Damkilde (2003) proposed another efficient lower-bound method, aimed primarily at solving structural engineering problems, based on non-linear programming.

Owing to the presence of singularities in their yield surfaces, where the gradients with respect to the stresses become undefined, the Tresca and Mohr-Coulomb criteria pose special difficulties in finite-element limit analysis. Lyamin \& Sloan (2002a) overcame this difficulty by local smoothing of the yield surface vertices, with an accompanying modification to the search direction to preserve feasibility during the optimisation iterations. An attractive alternative method for solving lower-bound limit analysis problems, which does not require differentiability of the yield surface in the optimisation process, is to use secondorder cone programming (Ciria, 2004; Makrodimopoulos \& Martin, 2006). This solution method can be applied to a variety of yield criteria in two dimensions, including the Tresca and Mohr-Coulomb models, and has proved to be robust and efficient for large-scale geotechnical problems (Krabbenhøft et al., 2007). In three-dimensional cases, second-order cone programming can be used for Von Mises and Drucker-Prager yield criteria, but not for Tresca or MohrCoulomb models. For the latter, which are of particular interest in geotechnical applications, it is possible to use a different cone-based solution algorithm that is known as semi-definite programming (Krabbenhøft et al., 2008). Like the second-order cone programming method, this approach does not require smoothing of any yield surface vertices, and it has proved to be both robust and efficient for largescale applications (Krabbenhøft et al., 2008). In summary, the second-order cone programming and semi-definite programming methods are, respectively, the solution methods of choice for the Tresca/Mohr-Coulomb models under twoand three-dimensional conditions. For yield criteria that are curved in the meridional plane, however, such as the HoekBrown model for rock, these procedures are inapplicable, and the more general interior point solution algorithm proposed by Lyamin \& Sloan (2002a) is appropriate.

Historical development of finite-element upper-bound analysis Early discrete formulations of the upper-bound theorem, based on finite elements and linear programming, were proposed by Anderheggen \& Knöpfel (1972) and Maier et al. (1972). Although quite general, these methods were concerned primarily with structural applications. The subsequent plane-strain procedures of Pastor \& Turgeman (1976) and Bottero et al. (1980), which focused on geotechnical applications with Tresca and Mohr-Coulomb yield criteria, permit a limited number of velocity discontinuities to occur between elements, but require the direction of shearing to be specified a priori. These formulations assume a piecewise linear velocity field using three-noded triangles, with each node having two unknown velocities, and each element being associated with a fixed number of unknown plastic multiplier rates. To ensure the solution is kinematically admissible, the velocities and plastic multiplier rates must satisfy a set of linear constraints arising from the flow rule, with the former unknowns also being subject to the appropriate boundary conditions. For a given set of prescribed velocities, the finite-element formulation optimises the velocities and plastic multiplier rates to minimise the power dissipated internally minus the rate of work done by fixed external forces. Once this quantity is known, it can be equated to the power expended by the external loads to furnish a strict upper bound on the true limit load. To generate a linear programming problem with an upper-bound finite-element formulation, it is again necessary to linearise the yield criterion. The polyhedral approximation must be external to the parent yield surface to ensure a rigorous upper bound, and each face of the linearised surface is associated with a single plastic multiplier. After assembling all the element and nodal contributions, the power dissipation in the triangles and the discontinuities, denoted by the quantities $\boldsymbol{c}_{1}^{\mathrm{T}} \boldsymbol{u}$ and $\boldsymbol{c}_{2}^{\mathrm{T}} \dot{\boldsymbol{\lambda}}$, minus the rate of work done by any fixed external forces, denoted by $\boldsymbol{c}_{3}^{\mathrm{T}} \boldsymbol{u}$, is minimised by solving a linear programming problem of the form

$$
\begin{array}{ll}
\boldsymbol{c}_{1}^{\mathrm{T}} \boldsymbol{u}+\boldsymbol{c}_{2}^{\mathrm{T}} \dot{\boldsymbol{\lambda}}-\boldsymbol{c}_{3}^{\mathrm{T}} \boldsymbol{u} & \begin{array}{l}
\text { power dissipation minus rate of } \\
\text { work done by fixed external forces }
\end{array}
\end{array}
$$

subject to

$$
\begin{aligned}
& \mathbf{A}_{11} \boldsymbol{u}+\mathbf{A}_{12} \dot{\boldsymbol{\lambda}}=\boldsymbol{0} \text { continuum flow rule } \\
& \mathbf{A}_{2} \boldsymbol{u} \quad=\mathbf{0} \text { discontinuity flow rule } \\
& \mathbf{A}_{3} \boldsymbol{u} \quad=\boldsymbol{b}_{3} \text { velocity boundary conditions } \\
& \mathbf{A}_{4} \boldsymbol{u} \quad \leqslant \mathbf{0} \text { discontinuity signs } \\
& \dot{\lambda} \geqslant 0 \text { plastic multiplier }
\end{aligned}
$$

where $\boldsymbol{c}_{1}, \boldsymbol{c}_{2}, \boldsymbol{c}_{3}$ and $\boldsymbol{b}_{3}$ are vectors of constants; $\mathbf{A}_{11}, \mathbf{A}_{12}$, $\mathbf{A}_{2}, \quad \mathbf{A}_{3}$ and $\mathbf{A}_{4}$ are matrices of constants; $\boldsymbol{u}$ is a global vector of nodal velocities; and $\dot{\lambda}$ is a global vector of element plastic multipliers.

Following these early procedures that focused on plane problems, Turgeman \& Pastor (1982) extended the upperbound formulation of Bottero et al. (1980) to handle axisymmetric geometries, but only for Von Mises and Tresca materials.

While the above upper-bound methods inherit all the key advantages of the finite-element technique, and hence can model complex problems in two dimensions, they were not widely applied in practice because of the CPU time required to solve their associated linear programming problems. In an effort to rectify this handicap, Sloan (1989) proposed an upper-bound method based on the steepest-edge active set solution scheme (Sloan, 1988b), which had proved successful for lower-bound limit analysis. Although it still suffered from the shortcoming of having to specify the direction of shearing along the velocity discontinuities a priori, the resulting method was subsequently used to generate useful upper bounds for a variety of underground structures including trapdoors (Sloan et al., 1990) and tunnels (Assadi \& Sloan, 1991; Sloan \& Assadi, 1991, 1992, 1994). Owing to the nature of the algorithm used to solve the associated linear optimisation problem, however, the procedure proved to be inefficient for large-scale examples involving thousands of elements.

Most early discrete formulations of the upper-bound theorem employed the three-noded triangle with a linearised yield function, since this leads to an optimisation problem with 
linear constraints where the power dissipation can be expressed solely in terms of the element plastic multipliers. By using an element with a constant-strain field, it is sufficient to enforce the flow rule over each triangle to define a kinematically admissible velocity field. Additional flow rule constraints, of course, are needed to define kinematically admissible velocity jumps across each discontinuity. If discontinuities are not included in a mesh of three-noded triangles, the elements should be arranged so that four triangles form a quadrilateral, with the central node lying at the intersection of the diagonals. Failing to observe this rule for undrained (incompressible) problems may lead to 'locking', where the elements cannot provide enough degrees of freedom to satisfy the constant-volume condition (Nagtegaal et al., 1974). In response to this shortcoming, Yu et al. (1994) developed a six-noded linear strain triangle for upperbound limit analysis. This element can model a velocity field accurately with fewer elements than the constant-strain triangle and, in the absence of discontinuities, no special grid arrangement is required for incompressible deformation.

The need to specify both the location and the direction of shearing for each discontinuity in an upper-bound analysis is a significant drawback, since it requires a good guess of the likely collapse mechanism in advance. This shortcoming was addressed by Sloan \& Kleeman (1995), who generalised the upper-bound formulation of Sloan (1989) to include velocity discontinuities at all edges shared by adjacent triangles. In their formulation, the direction of shearing is found as part of the optimisation process, and discontinuities are either active or inactive, depending on which deformation pattern gives the least amount of dissipated power. Each discontinuity is defined by four nodes, and requires four additional plastic multipliers to describe the normal and tangential velocity jumps along its length. The upper-bound procedure of Sloan \& Kleeman (1995) assumes a linearised yield criterion, and gives rise to a linear programming problem that can be solved using the active set solution algorithm of Sloan (1988b). It has proved to be computationally efficient for small- to medium-scale problems in two dimensions, and, because of the presence of velocity discontinuities at all shared element edges, gives good estimates of the limit load without the need for special grid arrangements. Examples where the method has provided useful upper bounds include slopes (Yu et al., 1998), foundations (Ukritchon et al., 1998; Merifield et al., 1999), anchors (Merifield et al., 2001, 2006a), and braced excavations (Ukritchon et al., 2003).

It is not straightforward to develop discontinuous upperbound formulations that can model an arbitrary yield condition. This is because the internal power dissipation depends on the state of stress as well as on the strain rates, so that in addition to finding the velocity and plastic multiplier fields that satisfy the flow rule, it is also necessary to compute a stress field that satisfies the yield criterion. Moreover, kinematically admissible discontinuities are difficult to incorporate at all inter-element edges in three dimensions.

The plate formulation described by Hodge \& Belytschko (1968) was one of the first attempts to develop a finiteelement upper-bound method based on non-linear programming. Their analysis used classical theory to specify the deformation field solely by the velocity normal to the original middle surface of the plate. This normal velocity was approximated within each element by a second-order polynomial, composed of independent nodal parameters, and the total internal power included contributions from plastic deformation through the elements, across hinge lines between elements and along clamped boundaries. The resulting unconstrained optimisation problem requires the ratio of the internal and external energy dissipation rates to be minimised, and was solved using the simplex method of Nelder \& Mead
(1965). Hodge \& Belytschko (1968) reported slow convergence of the procedure, owing to the complex nature of the objective function. Following this initial work, various other non-linear programming formulations were proposed for computing upper bounds on the load capacity of plates, shells and structures (Biron \& Charleux, 1972; Nguyen et al., 1978).

Huh \& Yang (1991) developed a general upper-bound procedure for plane stress problems using triangular elements with a linear velocity field. Their method focused on a socalled ' $\beta$-norm' family of yield criteria, which includes the von Mises model as a special case, and the results suggest that it is accurate and efficient for relatively large twodimensional problems. In a further development, Capsoni \& Corradi (1997) proposed another discrete upper-bound approach where the straining modes are modelled independently of rigid-body motions. This allows finite elements that are not involved in the collapse mechanism to be omitted from the dissipated power summation, and avoids problems with non-differentiability of the upper-bound functional.

In a different non-linear approach, Jiang (1994) proposed an upper-bound formulation, based on a regularised model of limit analysis (Friaâ, 1979), which assumes the material is visco-plastic, and uses two parameters to characterise its creep behaviour. By fixing the first of these parameters to unity, and letting the second one tend to infinity, it can be shown that the visco-plastic power dissipation converges to the plastic power dissipation, and a rigorous upper bound is obtained. Although this is an indirect method, the viscoplastic functional is always convex, even for three-dimensional Mohr-Coulomb and von Mises yield criteria, and there is always a unique solution that minimises it. To solve the resulting non-linear optimisation problem, Jiang (1994) employed the augmented Lagrangian method in conjunction with the algorithm of Uzawa (Fortin \& Glowinski, 1983). In a later paper, Jiang (1995) established that the same nonlinear programming scheme can be applied to perform upper-bound limit analysis directly. Jiang's formulations perform well for a variety of two-dimensional examples, but have not been extended to deal with discontinuities in the velocity field or three-dimensional geometries. Parallel to this development, Liu et al. (1995) proposed a direct iterative method for performing three-dimensional upper-bound limit analysis. This scheme treats the rigid zones separately from the plastic zones during each iteration, and neatly avoids the numerical difficulties that stem from a nondifferentiable objective function in the former. Their paper suggests that the process is efficient and numerically stable, and can be implemented easily in an existing displacement finite-element code. It has not, however, been widely used to generate rigorous upper bounds for geotechnical problems.

Following in the footsteps of their successful lower-bound formulation, Lyamin \& Sloan (2002b) developed an upperbound finite-element method that was also based on non-linear programming. This procedure assumes that the velocities vary linearly over each element, and that each element is associated with a constant-stress field and a single plastic multiplier rate. Flow rule constraints are imposed on the nodal velocities, element plastic multipliers and element stresses to ensure that the solution is kinematically admissible. In addition, to satisfy the consistency condition, the element stresses are constrained to obey the yield criterion, and the plastic multipliers are constrained to be non-negative. Using the approach developed in Sloan \& Kleeman (1995), the formulation of Lyamin \& Sloan (2002b) allows velocity discontinuities along shared element edges, with the velocity jumps across each discontinuity being defined by additional non-negative unknowns (plastic multipliers). Their procedure appears to be the first rigorous upper-bound method that incorporates both continuum and discontinuity deformation 
in two and three dimensions. Although the yield behaviour in the discontinuities is restricted to models with a linear yield envelope (e.g. Tresca and Mohr-Coulomb), it is otherwise quite general. The resulting optimisation problem can be solved in terms of the nodal velocities and element stresses alone by applying a two-stage, quasi-Newton algorithm directly to the Kuhn-Tucker optimality conditions (Lyamin \& Sloan, 2002b). Consequently, the element plastic multipliers do not need to be included explicitly as variables. This formulation has been used to compute accurate upper bounds for a wide range of important geotechnical problems, including sinkholes (Augarde et al., 2003a), tunnels (Lyamin \& Sloan, 2000), mines (Augarde et al., 2003b), foundation bearing capacity (Shiau et al., 2003; Hjiaj et al., 2004, 2005; Salgado et al., 2004) and anchors (Merifield et al., 2003).

Krabbenhøft et al. (2005) modified the upper-bound formulation of Lyamin \& Sloan (2002b) by proposing a new stress-based method that uses patches of continuum elements to incorporate velocity discontinuities in two and three dimensions. The elements in these patches have zero thickness, with opposing nodal pairs having the same coordinates, and the scheme results in a simple and efficient structure for programming. Moreover, the procedure can accommodate yield criteria that have curved envelopes, such as the HoekBrown model. Interestingly, the same idea can also be used to incorporate stress discontinuities in discrete formulations of lower-bound limit analysis (Lyamin et al., 2005a).

To avoid the problems associated with non-smooth yield surfaces in the optimisation process, second-order cone programming can be used to solve discrete formulations of the upper-bound theorem (Ciria, 2004; Makrodimopoulos \& Martin, 2007). This class of solution scheme is highly effective for lower-bound formulations, as discussed previously, and has proved to be equally effective for upper-bound formulations. Indeed, second-order cone programming is the method of choice for solving the optimisation problems that arise from finite-element upper-bound formulations, provided the yield function can be expressed in a conic quadratic form (such as the von Mises/Drucker-Prager model in plane strain or three dimensions, and the Tresca/Mohr-Coulomb model in plane strain). For the Tresca/Mohr-Coulomb model in three dimensions, the resulting upper-bound optimisation problems can be solved efficiently using semi-definite programming, just as in the lower-bound case.

\section{FINITE-ELEMENT LOWER-BOUND FORMULATION}

An efficient formulation of the lower-bound method will now be briefly described. This section follows the formula- tion of Lyamin \& Sloan (2002a), with some important modifications to handle stress discontinuities, and highlights the fundamental differences between finite-element limit analysis and displacement finite-element analysis. It also illustrates the power of using a discrete formulation of the classical limit theorems.

Figure 7 shows a soil mass, with volume $V$ and boundary area $A$, subject to a set of fixed surface stresses (tractions) $t$ acting on the boundary $A_{t}$, as well as an unknown set of tractions $\boldsymbol{q}$ acting on the boundary $A_{q}$. In practice $\boldsymbol{t}$ might correspond, for example, to a prescribed surcharge while $\boldsymbol{q}$ might correspond to an unknown bearing capacity. Also shown in Fig. 7 is a system of fixed body forces $g$ and unknown body forces $\boldsymbol{h}$ acting over the volume $V$. The former is typically a prescribed unit weight, while the latter, which corresponds to an unknown body force capacity, will be shown to be very useful in computing the stability of slopes, tunnels and excavations.

Recalling the problem solved earlier in the section 'Limit analysis', a lower-bound calculation seeks to find a statically admissible stress field $\boldsymbol{\sigma}=\left\{\sigma_{x x}, \sigma_{y y}, \sigma_{z z}, \tau_{x y}, \tau_{y z}, \tau_{x z}\right\}^{T}$ that satisfies equilibrium throughout $V$, balances the prescribed tractions $\boldsymbol{t}$ on $A_{t}$, nowhere violates the yield criterion $f$ so that $f(\boldsymbol{\sigma}) \leqslant 0$, and maximises the collapse load

$$
Q=\int_{A_{q}} Q_{1}(\boldsymbol{q}) \mathrm{d} A+\int_{V} Q_{2}(\boldsymbol{h}) \mathrm{d} V
$$

In the above, the functions $Q_{1}$ and $Q_{2}$ depend on the case at hand. For example, in a bearing capacity problem, $Q_{2}=0$, and one typically wants to maximise the load carried by the tractions normal to a boundary edge, $q_{\mathrm{n}}$, so that $Q_{1}=q_{\mathrm{n}}$ and

$$
Q=\int_{A_{q}} q_{\mathrm{n}} \mathrm{d} A
$$

For slope, tunnel and excavation problems, on the other hand, one often wants to maximise a dimensionless stability number that is a function of the soil unit weight $\gamma$. In this case $Q_{1}=0$ and $Q_{2}=\gamma$, giving

$$
Q=\int_{V} \gamma \mathrm{d} V
$$

where $\gamma$ is a variable which can be optimised.

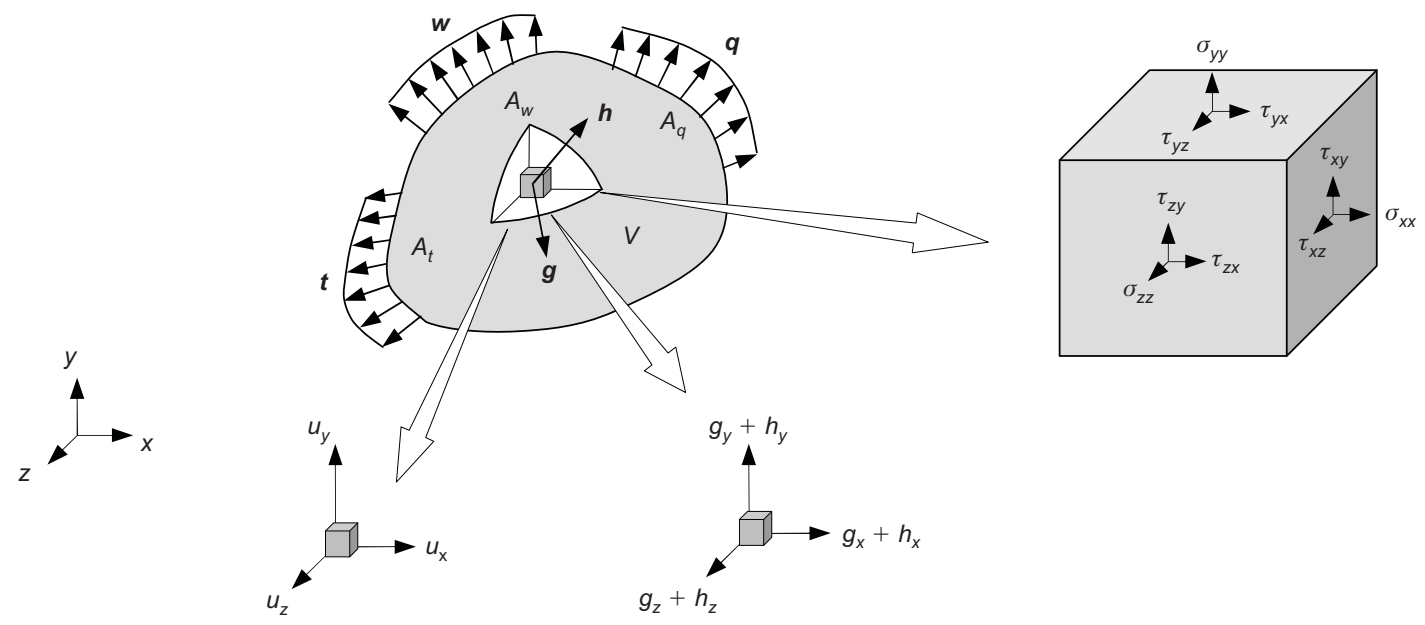

Fig. 7. Surface and body forces acting on soil mass 
Lower-bound finite elements

Following Lyamin \& Sloan (2002a), linear elements are used to discretise the domain. These elements, shown in Fig. 8 , enable a statically admissible stress field to be found in a rigorous manner, and have proved to be highly effective in large-scale applications.

The lower bound is found by formulating and solving a non-linear optimisation problem, where the nodal stresses and/or element body forces are the unknowns, and the objective function to be maximised corresponds to the collapse load. The unknowns are subject to equilibrium equality constraints for each continuum element, equilibrium equality constraints for each discontinuity, stress boundary conditions, and a yield condition inequality constraint for each node.

Figure 9 shows a very simple lower-bound mesh for the strip footing problem considered previously in Fig. 1. In this mesh, each node $i$ is associated with a vector of three unknown stresses, and each element $e$ is associated with a vector of two unknown body forces (which are not used in this example, but are included for the sake of generality). Owing to the presence of stress discontinuities between all inter-element edges, multiple nodes may share the same coordinates, and each node is unique to an element. Across each stress discontinuity, the normal and shear stresses are continuous. To satisfy the stress boundary conditions indicated, the nodal stresses along the corresponding edges in the grid are subject to appropriate equality constraints (using the standard stress transformation relations), and the stresses at each node $i$ in the grid, $\boldsymbol{\sigma}^{i}$, are subject to the yield condition $f\left(\boldsymbol{\sigma}^{i}\right) \leqslant 0$. The load to be maximised, given by

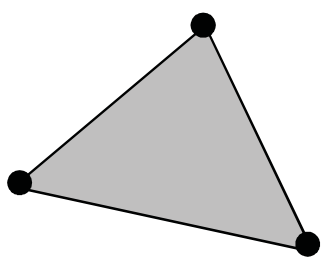

Node $\boldsymbol{\sigma}^{i}=\left\{\sigma_{x x}^{i}, \sigma_{y y}^{i}, \tau_{x y}^{i}\right\}^{\top}$

Element $\boldsymbol{h}^{e}=\left\{h_{x}^{e}, h_{y}^{e}\right\}^{\top}$

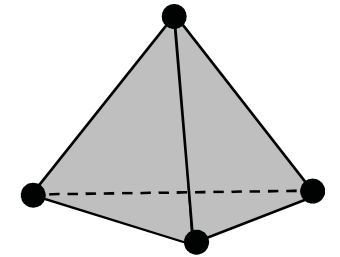

Node $\sigma^{i}=\left\{\sigma_{x x}^{i}, \sigma_{y y}^{i}, \sigma_{z z}^{i}, \tau_{x y}^{i}, \tau_{y z}^{i}, \tau_{x z}^{i}\right\}^{\top}$

Element $\boldsymbol{h}^{e}=\left\{h_{x}^{e}, h_{y}^{e}, h_{z}^{e}\right\}^{\top}$
Fig. 8. Linear elements for lower-bound limit analysis

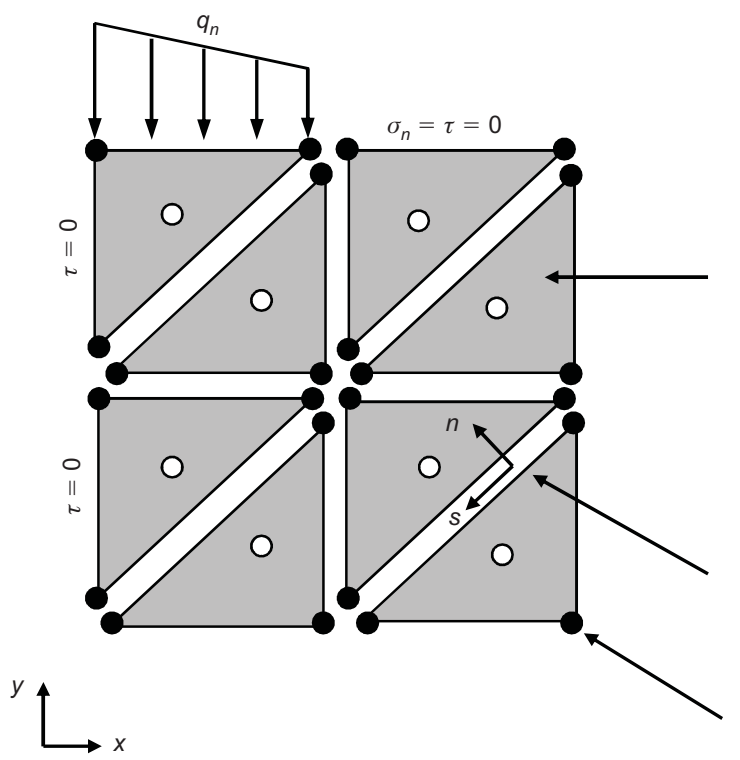

$$
\begin{aligned}
& \text { Maximise } Q=\int q_{n} \mathrm{~d} A=\text { collapse load } \\
& \text { Nodes } \sigma^{i}=\left\{\sigma_{x x}^{i}, \sigma_{y y}^{i}, \tau_{x y}^{i}\right\}^{\top} \\
& \text { Triangles } h^{e}=\left\{h_{x}^{e}, h_{y}^{e}\right\}^{\top}
\end{aligned}
$$

Stresses in triangles satisfy equilibrium

$$
\begin{aligned}
& \frac{\partial \sigma_{x x}}{\partial x}+\frac{\partial \tau_{x y}}{\partial y}+h_{x}+g_{x}=0 \\
& \frac{\partial \sigma_{y y}}{\partial y}+\frac{\partial \tau_{x y}}{\partial x}+h_{y}+g_{y}=0
\end{aligned}
$$

Stresses in discontinuities satisfy equilibrium

$\sigma_{n n}$ and $\tau_{n s}$ continuous for adjacent elements

Stresses at nodes satisfy yield condition $f\left(\boldsymbol{\sigma}^{j}\right) \leqslant 0$

Fig. 9. Illustrative lower-bound mesh for strip footing problem 
mass per unit thickness. Typically, one of these quantities is maximised, but it is also possible to maximise a force resultant in a specified direction. Using equations (5) and (6), and summing over all the loaded edges, the objective function (collapse load) can be expressed as

$$
\boldsymbol{c}_{1}^{\mathrm{T}} \boldsymbol{\sigma}
$$

where $c_{1}$ is a vector of constants, and $\boldsymbol{\sigma}$ is the global vector of unknown nodal stresses. At first sight, it would appear that the above approach is restricted to problems with linear geometry and linear loading. This limitation can be relaxed, however, by using the approach of Lyamin \& Sloan (2002a), which uses a local coordinate system.

For body loads $\boldsymbol{h}^{e}=\left\{h_{x}^{e}, h_{y}^{e}\right\}^{\mathrm{T}}$ acting on an element of area $A^{e}$ and unit thickness, the corresponding resultant forces, $Q_{x}=A^{e} h_{x}^{e}$ and $Q_{y}=A^{e} h_{y}^{e}$, are shown in Fig. 11. These forces may be assembled over the grid to give the total load produced as

$$
\boldsymbol{c}_{2}^{\mathrm{T}} \boldsymbol{h}
$$

where $\boldsymbol{c}_{2}$ is a vector of constants (element areas) and $\boldsymbol{h}$ is a global vector of element body loads. In practice, the most common case of body force optimisation involves a variable unit weight $\gamma$ so that $\boldsymbol{h}^{e}=\{0,-\gamma\}^{\mathrm{T}}$. For pseudo-dynamic stability analysis, however, it is also useful to be able to optimise the lateral body force component $h_{x}^{e}$.

\section{Continuum equilibrium}

In order to be statically admissible, the stresses in each element must satisfy the equilibrium equations

$$
\begin{aligned}
& \frac{\partial \sigma_{x x}}{\partial x}+\frac{\partial \tau_{x y}}{\partial y}+h_{x}+g_{x}=0 \\
& \frac{\partial \sigma_{y y}}{\partial y}+\frac{\partial \tau_{x y}}{\partial x}+h_{y}+g_{y}=0
\end{aligned}
$$

Over each triangle, the stresses vary linearly according to the relations

$$
\boldsymbol{\sigma}=\sum_{i=1}^{3} N_{i} \boldsymbol{\sigma}^{i}
$$

where $N_{i}$ are linear shape functions that are dependent on $x$ and $y$ and the element nodal coordinates. Inserting equation (8) into equation (7) yields the pair of equilibrium equations

$$
\left[\begin{array}{lll}
\mathbf{B}_{1}^{\mathrm{T}} & \mathbf{B}_{2}^{\mathrm{T}} & \mathbf{B}_{3}^{\mathrm{T}}
\end{array}\right] \boldsymbol{\sigma}^{e}=-\left(\boldsymbol{h}^{e}+\boldsymbol{g}^{e}\right)
$$

where the terms $\mathbf{B}_{i}$ are the standard strain-displacement (compatibility) matrices, defined by

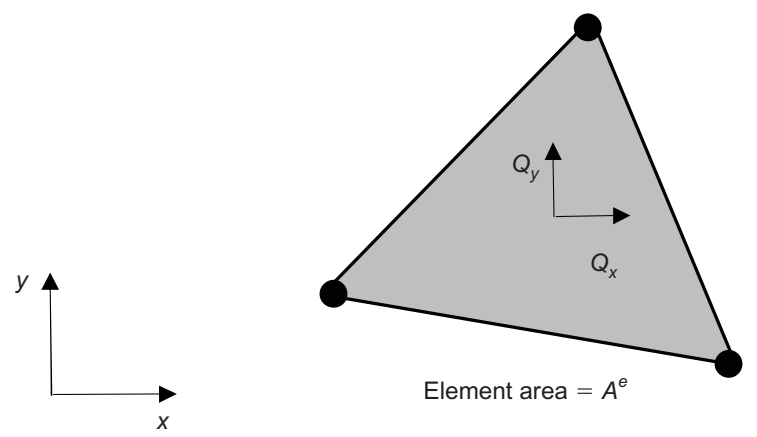

Fig. 11. Optimising body forces over an element

$$
\mathbf{B}_{i}^{\mathrm{T}}=\frac{1}{A^{e}}\left[\begin{array}{ccc}
b_{i} & 0 & c_{i} \\
0 & c_{i} & b_{i}
\end{array}\right]
$$

and $b_{i}$ and $c_{i}$ are constants that depend on the nodal coordinates. Rather than impose the equilibrium constraints in their native form, it is convenient to multiply both sides of equation (9) by the element area $A^{e}$. This permits an elegant implementation of stress discontinuities, as described in the next section, and leads to the modified equilibrium relations

$$
\left[\begin{array}{lll}
\overline{\mathbf{B}}_{1}^{\mathrm{T}} & \overline{\mathbf{B}}_{2}^{\mathrm{T}} & \overline{\mathbf{B}}_{3}^{\mathrm{T}}
\end{array}\right] \boldsymbol{\sigma}^{e}=-\left(\boldsymbol{h}^{e}+\boldsymbol{g}^{e}\right) A^{e}
$$

where

$$
\overline{\mathbf{B}}_{i}^{\mathrm{T}}=A^{e} \mathbf{B}_{i}^{\mathrm{T}}=\left[\begin{array}{ccc}
b_{i} & 0 & c_{i} \\
0 & c_{i} & b_{i}
\end{array}\right]
$$

Imposing the constraints in equation (11) ensures that the stresses satisfy the equilibrium conditions at every point in an element, thus satisfying a key requirement of the lowerbound theorem.

\section{Discontinuity equilibrium}

Stress discontinuities can dramatically improve the accuracy of the collapse load obtained from lower-bound calculations, and are introduced along all inter-element edges. Following the formulation of Lyamin et al. (2005a), each discontinuity is modelled by a patch of continuum elements of zero thickness, with opposing nodal pairs having the same coordinates, as shown in Fig. 12.

To satisfy equilibrium, and thus be statically admissible, the normal and shear stresses must be the same on both sides of the discontinuity according to the relations

$$
\left\{\begin{array}{c}
\sigma_{n n}^{1} \\
\tau_{n s}^{1}
\end{array}\right\}=\left\{\begin{array}{c}
\sigma_{n n}^{2} \\
\tau_{n s}^{2}
\end{array}\right\},\left\{\begin{array}{c}
\sigma_{n n}^{3} \\
\tau_{n s}^{3}
\end{array}\right\}=\left\{\begin{array}{c}
\sigma_{n n}^{4} \\
\tau_{n s}^{4}
\end{array}\right\}
$$

where for node $i$

$$
\left\{\begin{array}{c}
\sigma_{n n}^{i} \\
\tau_{n s}^{i}
\end{array}\right\}=\left[\begin{array}{rrr}
\cos ^{2} \beta & \sin ^{2} \beta & \sin 2 \beta \\
-\frac{1}{2} \sin 2 \beta & \frac{1}{2} \sin 2 \beta & \cos 2 \beta
\end{array}\right]\left\{\begin{array}{c}
\sigma_{x x}^{i} \\
\sigma_{y y}^{i} \\
\tau_{x y}^{i}
\end{array}\right\}
$$

Equations (13) and (14) imply that each pair of nodes on a stress discontinuity must obey two equality constraints on their associated Cartesian stresses. Summing these constraints over all nodal pairs on the discontinuities gives the global set of conditions that must be satisfied for discontinuity equilibrium.

Since equation (11) holds true for any value of the element area, it is possible to set $\delta \rightarrow 0$ for the triangles $D_{1}$ and $D_{2}$ in Fig. 12, so that $\left(x_{1}, y_{1}\right)=\left(x_{2}, y_{2}\right)$ and $\left(x_{3}, y_{3}\right)=\left(x_{4}, y_{4}\right)$. Considering triangle $D_{1}$, it can be shown that the equilibrium relations in equation (11) then become
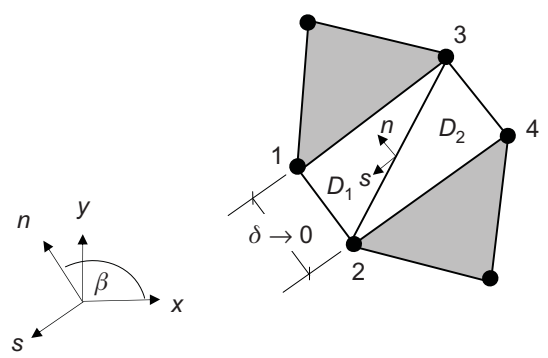

$\left(x_{1}, y_{1}\right)=\left(x_{2}, y_{2}\right)$ $\left(x_{3}, y_{3}\right)=\left(x_{4}, y_{4}\right)$

Fig. 12. Statically admissible stress discontinuity 


$$
\left[\begin{array}{lll}
\overline{\mathbf{B}}_{1}^{\mathrm{T}} & -\overline{\mathbf{B}}_{1}^{\mathrm{T}} & \mathbf{0}
\end{array}\right] \boldsymbol{\sigma}^{e}=\left[\begin{array}{lll}
\overline{\mathbf{B}}_{1}^{\mathrm{T}} & -\overline{\mathbf{B}}_{1}^{\mathrm{T}} & \mathbf{0}
\end{array}\right]\left\{\begin{array}{lll}
\boldsymbol{\sigma}^{1} & \boldsymbol{\sigma}^{2} & \boldsymbol{\sigma}^{3}
\end{array}\right\}^{\mathrm{T}}=\mathbf{0}
$$

which implies

$$
\overline{\mathbf{B}}_{1}^{\mathrm{T}} \boldsymbol{\sigma}^{1}=\overline{\mathbf{B}}_{1}^{\mathrm{T}} \boldsymbol{\sigma}^{2}
$$

Hence the left pair of relations in equation (13) are satisfied. A similar argument for triangle $D_{2}$ yields the right pair of relations in equation (13), so that all four of the discontinuity equilibrium conditions (equation (13)) are satisfied. Although the normal and shear stresses are continuous along each discontinuity, the tangential normal stress $\sigma_{s s}$ may jump, which means that the stresses can potentially differ at nodes that share the same coordinates. This type of formulation permits discontinuities to be modelled using standard continuum elements, and is simple to implement in both two and three dimensions. Other alternatives for implementing stress discontinuities are possible, such as imposing the constraints in equation (13) on the nodal stresses explicitly, and these have been used by a variety of researchers, including Pastor \& Turgeman (1976), Sloan (1988a) and Lyamin \& Sloan (2002a).

\section{Stress boundary conditions}

To satisfy equilibrium, the stresses for any boundary node must match the prescribed surface tractions (stresses) $\boldsymbol{t}$. These boundary conditions may be specified in a Cartesian reference frame, but are more commonly defined in terms of normal and tangential components along a boundary edge, as shown in Fig. 13.

Noting that the stresses vary linearly along an edge, the stress boundary conditions take the form

$$
\left\{\begin{array}{c}
\sigma_{n n}^{1} \\
\tau_{n s}^{1}
\end{array}\right\}=\left\{\begin{array}{c}
t_{n}^{1} \\
t_{s}^{1}
\end{array}\right\}, \quad\left\{\begin{array}{c}
\sigma_{n n}^{2} \\
\tau_{n s}^{2}
\end{array}\right\}=\left\{\begin{array}{c}
t_{n}^{2} \\
t_{s}^{2}
\end{array}\right\}
$$

where $\left\{\begin{array}{ll}\sigma_{n n}^{i}, & \tau_{n s}^{i}\end{array}\right\}^{\mathrm{T}}$ for node $i$ are again given by equation (14). These constraints must be applied to all edges where surface stresses are specified, and they ensure that the boundary conditions are satisfied exactly for a linear finiteelement model.

\section{Yield conditions}

Provided the stresses vary linearly over an element and the yield function $f(\boldsymbol{\sigma})$ is convex, the yield condition is satisfied at every point in the domain if the inequality constraint $f\left(\boldsymbol{\sigma}^{i}\right) \leqslant 0$ is imposed at each node $i$. In the twodimensional case, this implies that the nodal stresses for each triangle are subject to three non-linear inequality constraints, as shown in Fig. 14.
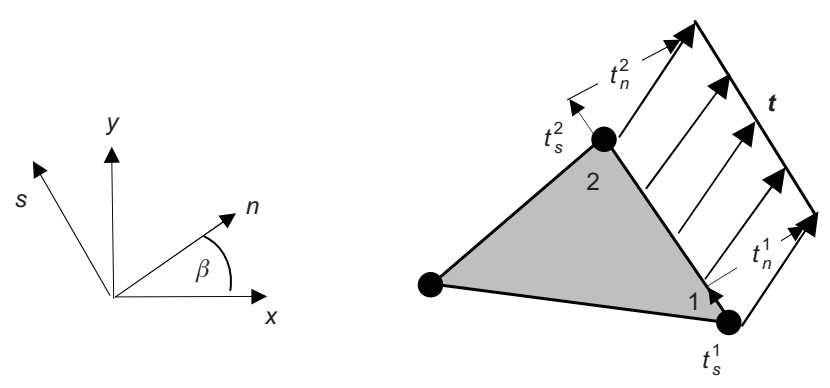

Fig. 13. Stress boundary conditions

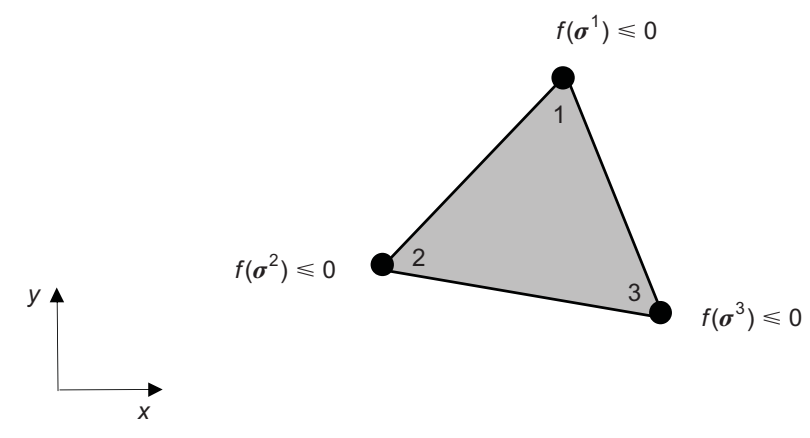

Fig. 14. Yield conditions

\section{Extension elements}

For problems involving semi-infinite domains, special 'extension' elements are needed to complete the stress field so that the equilibrium, stress boundary and yield conditions are satisfied everywhere. These elements are placed around the periphery of a standard mesh, and although their effect is often small for a grid that is sufficiently large to capture the zone of plastic yielding, they do guarantee that the solution is a rigorous lower bound. For two-dimensional applications where the yield surface has a linear envelope, complete stress fields can be found using the unidirectional and bidirectional extension elements shown in Fig. 15. The equilibrium and stress boundary conditions for these extension elements are identical to those for the standard continuum elements, with the only change being the different yield conditions (Pastor, 1978). In the latter, the function $F(\boldsymbol{\sigma})$ is defined by the relation $f(\boldsymbol{\sigma})=F(\boldsymbol{\sigma})-k$, where $k$ is a non-negative constant (Makrodimopoulos \& Martin, 2006).

For the unidirectional extension element, node 4 is a dummy node, since its stresses are not independent and can be expressed as linear combinations of the stresses at nodes 1 , 2 and 3. This node is included for the sole purpose of being able to accommodate a stress discontinuity along the edge defined by nodes 3 and 4 , and it is not subject to constraints other than those imposed by discontinuity equilibrium.

For cases where the envelope of the yield surface is not linear, such as the widely used Hoek-Brown criterion, the above extension elements are inapplicable, and hence the computed lower bounds are based on an 'incomplete' stress field. Although theoretically undesirable, this is not a serious shortcoming in practice, since the extension conditions seldom have a significant effect on the collapse load for a wellconstructed mesh that includes all the zones of plastic yielding.

\section{Lower-bound non-linear optimisation problem}

For a given mesh, summing the various objective function coefficients and constraints described above leads to the non-

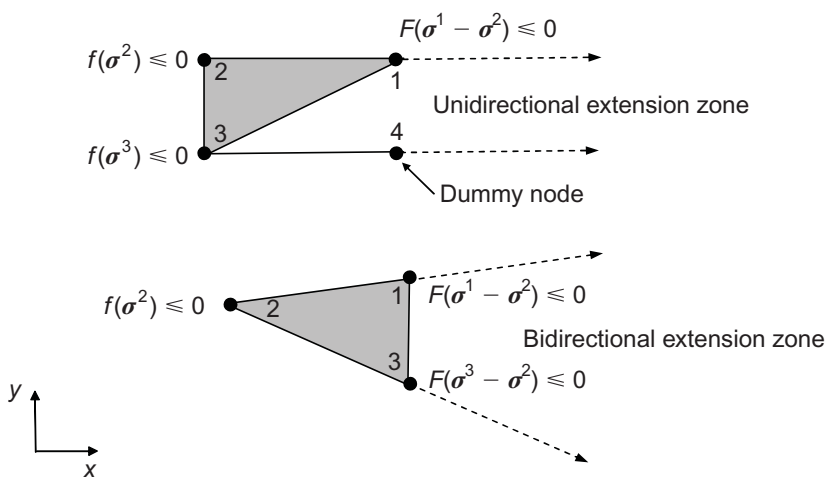

Fig. 15. Extension elements 
linear optimisation problem in equation (3), where the unknowns are the nodal stresses $\boldsymbol{\sigma}$ and element body loads $\boldsymbol{h}$. The solution to this non-linear programming problem, which constitutes a statically admissible stress field, can be found efficiently by solving the system of non-linear equations that define its Kuhn-Tucker optimality conditions. Such a strategy was proposed by Lyamin (1999) and Lyamin \& Sloan (2002a), who developed a two-stage quasi-Newton solver that typically requires less than about 50 iterations, regardless of the problem size. Because it does not require the yield surface to be linearised, this type of approach is applicable in three dimensions for a wide range of smooth yield criteria, including those with curved envelopes in the meridional plane. As noted previously, however, for the Tresca and Mohr-Coulomb yield functions, the vertices must be smoothed to remove the singularities in the gradients and obtain good convergence. Alternatively, second-order cone programming and semi-definite programming algorithms are fast and efficient solution methods for the Tresca/MohrCoulomb models under two- and three-dimensional conditions respectively (Ciria, 2004; Makrodimopoulos \& Martin, 2006; Krabbenhøft et al., 2007). Both these procedures are applicable to non-smooth yield criteria, and are thus ideally suited to the Tresca and Mohr-Coulomb models.

\section{FINITE-ELEMENT UPPER-BOUND FORMULATION}

An efficient formulation of the finite-element upper-bound method will now be briefly outlined. This follows the formulation of Lyamin \& Sloan (2002b), with important modifications to handle velocity discontinuities as described by Krabbenhøft et al. (2005). Recalling the problem solved in the section 'Limit analysis' and with reference to Fig. 7, an upper-bound calculation searches for a velocity distribution $\boldsymbol{u}=\left\{u_{x}, u_{y}, u_{z}\right\}^{\mathrm{T}}$ that satisfies compatibility, the flow rule and the velocity boundary conditions $\boldsymbol{w}$ on the surface area $A_{w}$, and minimises the internal power dissipation (due to plastic shearing) less the rate of work done by the fixed external loads. Mathematically, the latter quantity can be written as

$$
\dot{W}=P_{\text {int }}-\int_{A_{t}} \boldsymbol{t}^{\mathrm{T}} \boldsymbol{u} \mathrm{d} A-\int_{V} \boldsymbol{g}^{\mathrm{T}} \boldsymbol{u} \mathrm{d} V
$$

where $P_{\text {int }}$ is the plastic dissipation, defined by

$$
P_{\text {int }}=\int_{V} \boldsymbol{\sigma}^{\mathrm{T}} \dot{\boldsymbol{\varepsilon}}^{\mathrm{p}} \mathrm{d} V
$$

and

$$
\dot{\varepsilon}^{\mathrm{p}}=\left\{\dot{\varepsilon}_{x x}^{\mathrm{p}}, \quad \dot{\varepsilon}_{y y}^{\mathrm{p}}, \quad \varepsilon_{z z}^{\mathrm{p}}, \quad \dot{\gamma}_{x y}^{\mathrm{p}}, \quad \dot{\gamma}_{y z}^{\mathrm{p}}, \quad \dot{\gamma}_{x z}^{\mathrm{p}}\right\}^{\mathrm{T}}
$$

are the plastic strain rates. An upper bound on the limit load is then found by equating the optimised value of $\dot{W}$ to the power expended by the external loads, which may be written as

$$
P_{\text {ext }}=\int_{A_{q}} \boldsymbol{q}^{\mathrm{T}} \boldsymbol{u} \mathrm{d} A+\int_{V} \boldsymbol{h}^{\mathrm{T}} \boldsymbol{u} \mathrm{d} V
$$

As in the lower-bound formulation, it is possible to optimise either the total force carried by the external tractions, $\boldsymbol{q}$, or some set of body forces $\boldsymbol{h}$ (typically the unit weight).

\section{Upper-bound finite elements}

Following Lyamin \& Sloan (2002b), linear elements are used to discretise the domain. These elements, shown in Fig. 16 , enable a kinematically admissible velocity field to be
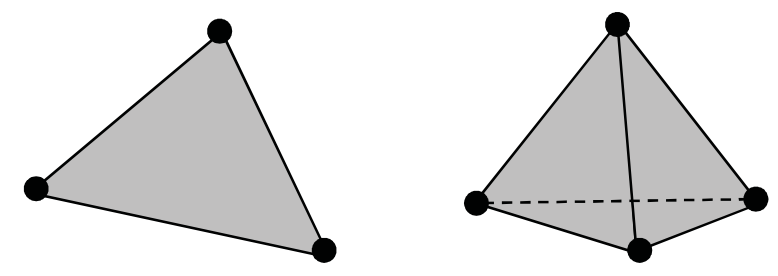

$\begin{array}{ll}\text { Node } \boldsymbol{u}^{i}=\left\{u_{x}^{i}, u_{y}^{i}\right\}^{\top} & \text { Node } \boldsymbol{u}^{i}=\left\{u_{x}^{i}, u_{y}^{i}, u_{z}^{i}\right\}^{\top} \\ \text { Element } \boldsymbol{\sigma}^{e}=\left\{\sigma_{x x}^{e}, \sigma_{y y}^{e}, \tau_{x y}^{e}\right\}^{\top} & \text { Element } \boldsymbol{\sigma}^{e}=\left\{\sigma_{x x}^{e}, \sigma_{y y}^{e}, \sigma_{z z}^{e}, \tau_{x y}^{e}, \tau_{y z}^{e}, \tau_{x z}^{e}\right\}^{\top}\end{array}$

Fig. 16. Linear elements for upper-bound limit analysis

found in a rigorous manner, and are combined with a patchbased method for modelling velocity discontinuities along all inter-element edges (Krabbenhøft et al., 2005). The procedure of Lyamin \& Sloan (2002b) was the first to incorporate velocity discontinuities in three dimensions, and has proved highly effective for solving large-scale stability problems in geotechnical engineering. The scheme of Krabbenhøft et al. (2005), which models each discontinuity by a patch of continuum elements of zero thickness, is particularly advantageous in three dimensions, is applicable to general types of yield criterion, and parallels the formulation described above for the lower-bound method. The elements shown in Fig. 16 adopt a linear variation of the velocities $\boldsymbol{u}$ together with a constant-stress field $\boldsymbol{\sigma}$. Although it is possible to develop discrete upper-bound formulations that do not include the stresses as unknowns, these are restricted to yield models with a linear envelope. Moreover, as discussed later, inclusion of the stresses provides a very convenient platform for developing a mesh refinement strategy, with an exact error estimate, for minimising the gap between the solutions from the upper- and lower-bound formulations.

The upper-bound procedure is formulated as a non-linear optimisation problem, where nodal velocities, element stresses and plastic multipliers are the unknowns, and the objective function to be minimised is the internal power dissipation less the rate of work done by fixed external forces. To satisfy the requirements of the upper-bound theorem, the unknowns are subject to constraints arising from the flow rule, the velocity boundary conditions and the yield condition.

Figure 17 shows a very simple upper-bound mesh for the strip footing problem considered previously in Fig. 1. Each node $i$ is associated with a vector of two unknown velocities, and each element $e$ is associated with a vector of three unknown stresses and an unknown non-negative plastic multiplier rate $\dot{\lambda}$. In each triangle the plastic strains (velocities) are subject to the constraints imposed by the associated flow rule, and also satisfy the consistency requirement $\dot{\lambda} f\left(\boldsymbol{\sigma}^{e}\right)=0$. The latter condition ensures that plastic deformation takes place only for points on the yield surface. Owing to the presence of velocity discontinuities along all inter-element edges, multiple nodes may share the same coordinates, and each node is unique to an element. In general, two plastic multipliers are used to model the normal and tangential velocity jumps in each of these discontinuities, and these are governed by the corresponding associated flow rule. To satisfy the velocity boundary conditions, the relevant nodal velocities on the periphery of the grid are subject to appropriate equality constraints. The upper bound on the limit load is found by using equations (15) and (17), noting that $\boldsymbol{t}=\boldsymbol{g}=\boldsymbol{h}=0$ for this case, to minimise $P_{\text {int }}$ and hence $Q$.

The key steps in formulating an efficient upper-bound method using finite elements are now outlined for the two- 


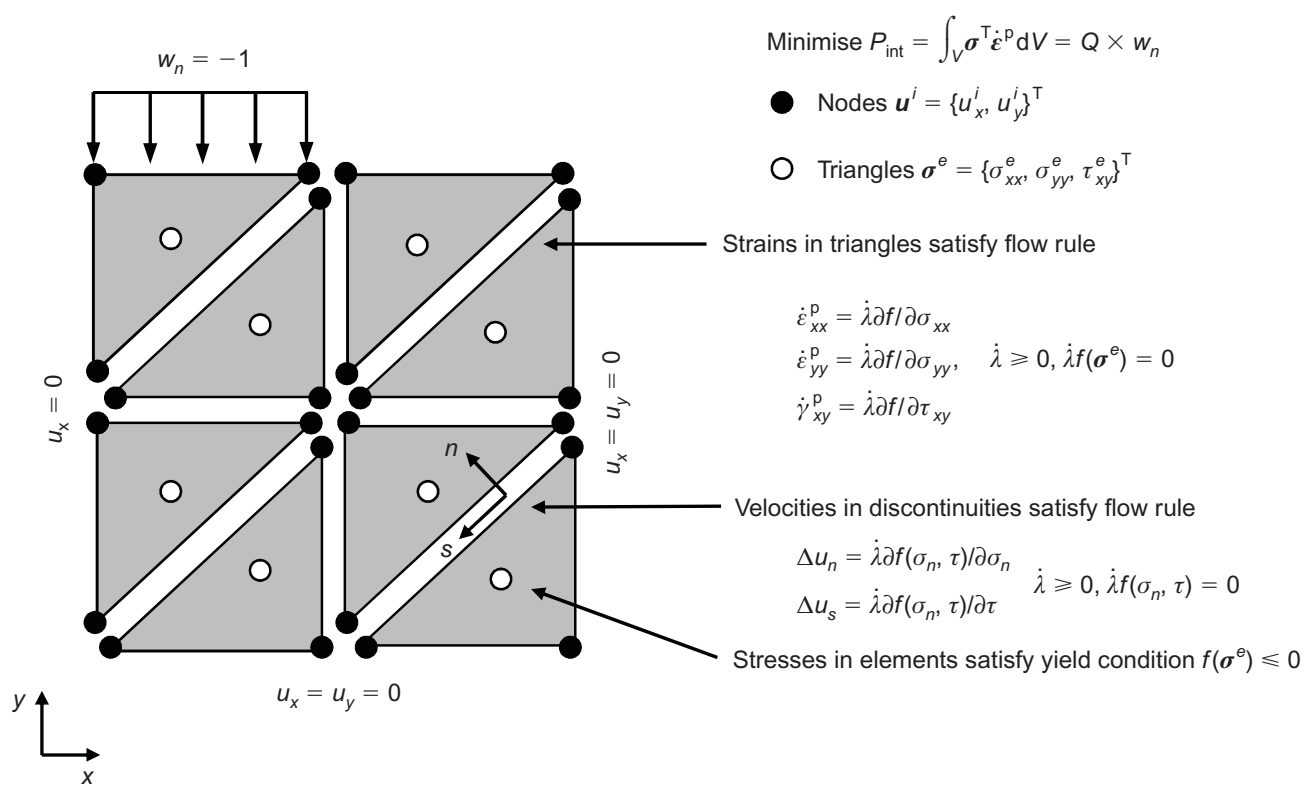

Fig. 17. Illustrative upper-bound mesh for strip footing problem

dimensional case. A similar formulation for three dimensions can be found in Lyamin \& Sloan (2002b).

\section{Objective function}

In the finite-element upper-bound formulation, the objective function corresponds to the internal rate of energy dissipated by plastic shearing less the energy expended by the fixed external forces, and it is given by equations (15) and (16). Noting that the stresses and plastic strain rates are constant over each element, and summing over all the elements, the internal power dissipation may be written as

$$
P_{\text {int }}=\int_{V} \boldsymbol{\sigma}^{\mathrm{T}} \dot{\boldsymbol{\varepsilon}}^{\mathrm{p}} \mathrm{d} V=\sum_{e}\left(\boldsymbol{\sigma}^{\mathrm{T}} \dot{\boldsymbol{\varepsilon}}^{\mathrm{p}} V\right)^{e}
$$

This quantity can be evaluated conveniently by observing that the (constant) plastic strain rates are related to the nodal velocities by the strain-displacement relations

$$
\dot{\boldsymbol{\varepsilon}}^{\mathrm{p}}=\mathbf{B}^{e} \boldsymbol{u}^{e}
$$

where for the linear triangle

$$
\mathbf{B}^{e}=\left[\begin{array}{lll}
\mathbf{B}_{1} & \mathbf{B}_{2} & \mathbf{B}_{2}
\end{array}\right], \boldsymbol{u}^{e}=\left\{u_{x}^{1}, u_{y}^{1}, u_{x}^{2}, u_{y}^{2}, u_{x}^{3}, u_{y}^{3}\right\}^{\mathrm{T}}
$$

and $\mathbf{B}_{i}$ is given by equation (10). Using the matrix $\overline{\mathbf{B}}^{e}=A^{e} \mathbf{B}^{e}$ defined in equation (12), equations (18) and (19) furnish the total internal dissipated power for the mesh, in terms of the unknowns stresses and velocities, as

$$
P_{\text {int }}=\boldsymbol{\sigma}^{\mathrm{T}} \overline{\mathbf{B}} \boldsymbol{u}
$$

where $\boldsymbol{\sigma}$ is a global vector of element stresses, $\boldsymbol{u}$ is a global vector of nodal velocities, and $\overline{\mathbf{B}}=\sum_{e} \overline{\mathbf{B}}^{e}$.

Using the formulation of Krabbenhøft et al. (2005), which models each discontinuity by a patch of continuum elements of zero thickness, equation (20) can also be used, without modification, to compute the plastic dissipation in the velocity discontinuities due to plastic shearing. Thus $P_{\text {int }}$ is found by summing over both the continuum elements and the discontinuity elements, as they can be treated identically.

The remaining two integrals in equation (15), involving the fixed tractions $\boldsymbol{t}$ and body forces $\boldsymbol{h}$, can be evaluated using the linear expansions for the velocities $\boldsymbol{u}$, and lead to an expression of the form

$$
\boldsymbol{c}^{\mathrm{T}} \boldsymbol{u}=\int_{A_{t}} \boldsymbol{t}^{\mathrm{T}} \boldsymbol{u} \mathrm{d} A+\int_{V} \boldsymbol{g}^{\mathrm{T}} \boldsymbol{u} \mathrm{d} V
$$

where $c$ is a vector of known constants. After combining equations (15), (20) and (21), the final objective function for the upper-bound formulation can be written as

$$
\boldsymbol{\sigma}^{\mathrm{T}} \overline{\mathbf{B}} \boldsymbol{u}-\boldsymbol{c}^{\mathrm{T}} \boldsymbol{u}
$$

\section{Continuum flow rule}

To give an upper bound on the limit load, the velocity field must be kinematically admissible and satisfy the constraints imposed by an associated flow rule. For the triangular element shown in Fig. 16, the flow rule conditions may be written as

$$
\begin{aligned}
& \dot{\varepsilon}_{x x}^{\mathrm{p}}=\dot{\lambda} \partial f / \partial \sigma_{x x} \\
& \dot{\varepsilon}_{y y}^{\mathrm{p}}=\dot{\lambda} \partial f / \partial \sigma_{y y}, \quad \dot{\lambda} \geqslant 0, \quad \dot{\lambda} f\left(\boldsymbol{\sigma}^{e}\right)=0 \\
& \dot{\gamma}_{x y}^{\mathrm{p}}=\dot{\lambda} \partial f / \partial \tau_{x y}
\end{aligned}
$$

or

$$
\dot{\boldsymbol{\varepsilon}}^{\mathrm{p}}=\dot{\lambda} \nabla f\left(\boldsymbol{\sigma}^{e}\right), \quad \dot{\lambda} \geqslant 0, \quad \dot{\lambda} f\left(\boldsymbol{\sigma}^{e}\right)=0
$$

where $\dot{\lambda}$ is the plastic multiplier. After combining equations (19) and (22), and then multiplying both sides by the element area, the flow rule constraints for each element may be expressed as

$$
\overline{\mathbf{B}}^{e} \boldsymbol{u}^{e}=\dot{\alpha} \nabla f\left(\boldsymbol{\sigma}^{e}\right), \quad \dot{\alpha} \geqslant 0, \quad \dot{\alpha} f\left(\boldsymbol{\sigma}^{e}\right)=0
$$

where $\dot{\alpha}=A^{e} \dot{\lambda}$ denotes the conventional plastic multiplier times the element area. Thus, for the two-dimensional case, the continuum flow rule generates four equality constraints and one inequality constraint on the element unknowns. Unless the yield criterion is a linear function of the stresses, all the equality constraints are non-linear.

\section{Discontinuity flow rule}

The patch-based formulation of Krabbenhøft et al. (2005) incorporates velocity discontinuities using a procedure identical to that mentioned previously for the lower-bound method. For the two-dimensional case, shown in Fig. 18, 


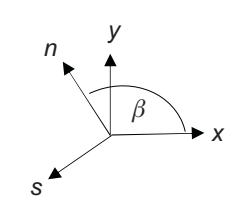

Area of $D_{1}$ and $D_{2}=\frac{1}{2} \delta \mathrm{L}$

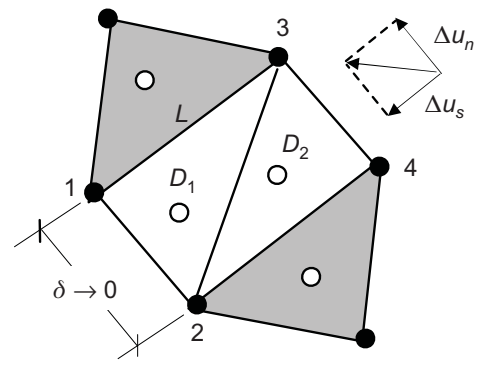

$\left(x_{1}, y_{1}\right)=\left(x_{2}, y_{2}\right)$

$\left(x_{3}, y_{3}\right)=\left(x_{4}, y_{4}\right)$

Fig. 18. Kinematically admissible velocity discontinuity

each discontinuity comprises two triangles of zero thickness, and thus has six unknown stresses. Both triangles are subject to the flow conditions defined by equations (23).

Across the discontinuity, velocity jumps can occur in the normal and tangential directions, so that the velocities can potentially differ at nodes that share the same coordinates. In the following, the implications of enforcing the flow rule conditions in equations (23) for zero-thickness elements are discussed, and it is shown that the resulting discontinuity formulation is equivalent to that proposed by Sloan \& Kleeman (1995).

Using the strain-displacement relations equations (10) and (19) with the discontinuity width $\delta \rightarrow 0$, it is straightforward to show that the local strains in triangle $D_{1}$ approach the values

$$
\begin{aligned}
& \dot{\varepsilon}_{s s}^{\mathrm{p}} \rightarrow 0 \\
& \dot{\varepsilon}_{n n}^{\mathrm{p}} \rightarrow \Delta u_{n}^{12} / \delta \\
& \dot{\gamma}_{n s}^{\mathrm{p}} \rightarrow \Delta u_{s}^{12} / \delta
\end{aligned}
$$

where

$$
\left(\Delta u_{n}^{12}, \Delta u_{s}^{12}\right)=\left(u_{n}^{1}-u_{n}^{2}, u_{s}^{1}-u_{s}^{2}\right)
$$

are the normal and tangential velocity jumps at the nodal pair $(1,2)$. From equation (24) it is clear that the strains become infinite as $\delta \rightarrow 0$, but multiplying them by the element area $0 \cdot 5 \delta L$ gives finite quantities according to

$$
\begin{aligned}
& A^{e} \dot{\varepsilon}_{s s}^{\mathrm{p}}=0 \\
& A^{e} \dot{\varepsilon}_{n n}^{\mathrm{p}}=\Delta u_{n}^{12} L / 2 \\
& A^{e} \dot{\gamma}_{n s}^{\mathrm{p}}=\Delta u_{s}^{12} L / 2
\end{aligned}
$$

where a unit out-of-plane element thickness has been assumed. Similar relations hold for triangle $D_{2}$, with the superscript pair $(1,2)$ being replaced by $(3,4)$. These relations confirm that discontinuous velocity jumps can be modelled by using two zero-thickness continuum elements with $\left(x_{1}, y_{1}\right)=\left(x_{2}, y_{2}\right)$ and $\left(x_{3}, y_{3}\right)=\left(x_{4}, y_{4}\right)$, provided the flow rule constraints (equations (23)) are satisfied over each triangle. Note that $\dot{\alpha}$ in equation (23) is well-defined as $\delta \rightarrow 0$, even though it is the product of a quantity that is zero $\left(A^{e}\right)$ and a quantity that is infinite $(\dot{\lambda})$. For a planestrain discontinuity, the general yield condition $f\left(\boldsymbol{\sigma}^{e}\right)$ can, without loss of generality, be replaced by its planar counterpart $f\left(\sigma_{n}, \tau\right)$, where $\sigma_{n}$ denotes $\sigma_{n n}$ and $\tau$ denotes $\tau_{n s}$. The flow rule conditions (equations (23)) that define $\left(\Delta u_{n}, \Delta u_{s}\right)$ are then given by

$$
\begin{aligned}
& \Delta u_{n}=\dot{\alpha} \partial f / \partial \sigma_{n} \\
& \Delta u_{s}=\dot{\alpha} \partial f / \partial \tau
\end{aligned}, \quad \dot{\alpha} \geqslant 0, \quad \dot{\alpha} f\left(\sigma_{n}, \tau\right)=0
$$

The common case of a velocity discontinuity in a MohrCoulomb material is shown in Fig. 19. Using Koiter's theorem for composite yield surfaces, the jumps in the normal and tangential directions are given by

$$
\begin{aligned}
& \Delta u_{n}=\Delta u_{n}^{+}+\Delta u_{n}^{-}=\left(\dot{\alpha}^{+}+\dot{\alpha}^{-}\right) \tan \phi \\
& \Delta u_{s}=\Delta u_{s}^{+}+\Delta u_{s}^{-}=\dot{\alpha}^{+}-\dot{\alpha}^{-}
\end{aligned}
$$

where

$$
\begin{aligned}
& \dot{\alpha}^{+} \geqslant 0 \\
& \dot{\alpha}^{-} \geqslant 0
\end{aligned}
$$

and

$$
\begin{aligned}
& \dot{\alpha}^{+} f^{+}\left(\sigma_{n}, \tau\right)=0 \\
& \dot{\alpha}^{-} f^{-}\left(\sigma_{n}, \tau\right)=0
\end{aligned}
$$

Noting that the normal and tangential jumps at a nodal pair $(i, j)$ can be expressed in terms of the Cartesian velocity jumps through the relations

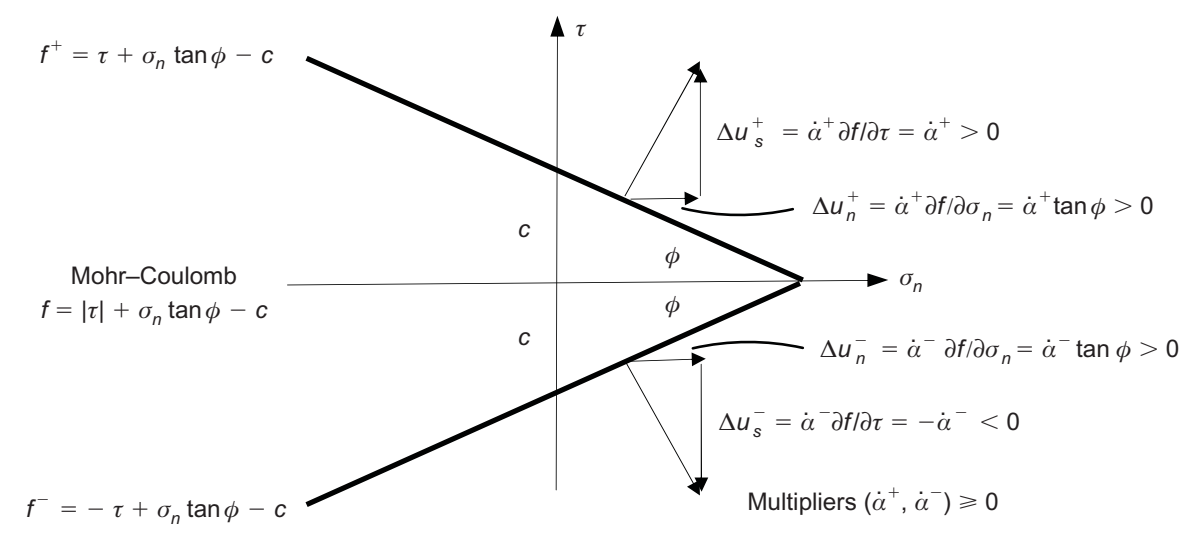

Fig. 19. Mohr-Coulomb yield criterion for velocity discontinuity 


$$
\left\{\begin{array}{l}
\Delta u_{n}^{i j} \\
\Delta u_{s}^{i j}
\end{array}\right\}=\left[\begin{array}{rr}
\cos \beta & \sin \beta \\
-\sin \beta & \cos \beta
\end{array}\right]\left\{\begin{array}{l}
\Delta u_{x}^{i j} \\
\Delta u_{y}^{i j}
\end{array}\right\}
$$

it follows that the complete set of flow rule constraints for the discontinuity is given by

$$
\begin{aligned}
& {\left[\begin{array}{rr}
\cos \beta & \sin \beta \\
-\sin \beta & \cos \beta
\end{array}\right]\left\{\begin{array}{l}
\Delta u_{x}^{12} \\
\Delta u_{y}^{12}
\end{array}\right\}=\left\{\begin{array}{c}
\left(\dot{\alpha}^{+12}+\dot{\alpha}^{-12}\right) \tan \phi \\
\dot{\alpha}^{+12}-\dot{\alpha}^{-12}
\end{array}\right\}} \\
& {\left[\begin{array}{rr}
\cos \beta & \sin \beta \\
-\sin \beta & \cos \beta
\end{array}\right]\left\{\begin{array}{c}
\Delta u_{x}^{34} \\
\Delta u_{y}^{34}
\end{array}\right\}=\left\{\begin{array}{c}
\left(\dot{\alpha}^{+34}+\dot{\alpha}^{-34}\right) \tan \phi \\
\dot{\alpha}^{+34}-\dot{\alpha}^{-34}
\end{array}\right\}} \\
& \dot{\alpha}^{+12} \geqslant 0 \\
& \dot{\alpha}^{-12} \geqslant 0 \\
& \dot{\alpha}^{+34} \geqslant 0 \\
& \dot{\alpha}^{-34} \geqslant 0 \\
& \dot{\alpha}^{+12} f^{+}\left(\sigma_{n}^{1}, \tau^{1}\right)=0 \\
& \dot{\alpha}^{-12} f^{-}\left(\sigma_{n}^{1}, \tau^{1}\right)=0 \\
& \dot{\alpha}^{+34} f^{+}\left(\sigma_{n}^{2}, \tau^{2}\right)=0 \\
& \dot{\alpha}^{-34} f^{-}\left(\sigma_{n}^{2}, \tau^{2}\right)=0
\end{aligned}
$$

where $\left(\dot{\alpha}^{+i j}, \dot{\alpha}^{-i j}\right)$ denotes the values of the plastic multipliers $\left(\dot{\alpha}^{+}, \dot{\alpha}^{-}\right)$at the nodal pair $(i, j),\left(\sigma_{n}^{1}, \tau^{1}\right)$ are the stresses in triangle $D_{1}$, and $\left(\sigma_{n}^{2}, \tau^{2}\right)$ are the stresses in $D_{2}$. Equations (25)-(27) are identical to the formulation proposed in Sloan \& Kleeman (1995), which uses line elements to model a velocity discontinuity without including element stresses. Thus imposing the constraints in equation (23) over the zero-thickness triangles $D_{1}$ and $D_{2}$ is sufficient to model a velocity discontinuity.

\section{Velocity boundary conditions}

To be kinematically admissible, the velocity field must satisfy the prescribed boundary conditions. These boundary conditions may be specified in a Cartesian reference frame but, as shown in Fig. 20, are more commonly defined in terms of normal and tangential velocity components along a boundary edge.

Noting that the velocities vary linearly along each edge, the general form of the boundary conditions may be expressed as

$$
\left\{\begin{array}{l}
u_{n}^{1} \\
u_{s}^{1}
\end{array}\right\}=\left\{\begin{array}{l}
w_{n}^{1} \\
w_{s}^{1}
\end{array}\right\}, \quad\left\{\begin{array}{l}
u_{n}^{2} \\
u_{s}^{2}
\end{array}\right\}=\left\{\begin{array}{l}
w_{n}^{2} \\
w_{s}^{2}
\end{array}\right\}
$$

where, for some node $i$, the transformed nodal velocities are related to the Cartesian velocities by the standard equations
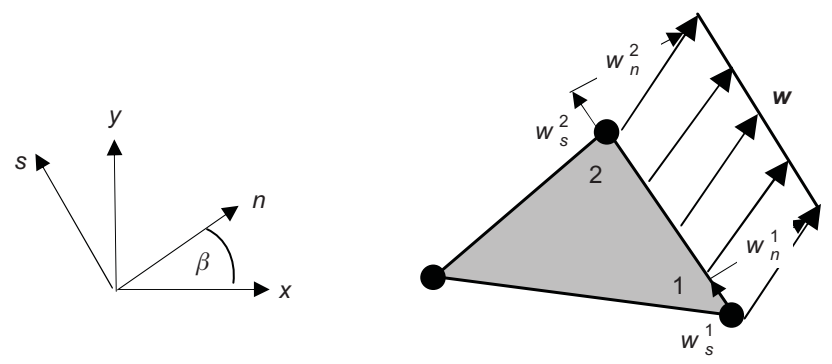

Fig. 20. Velocity boundary conditions

$$
\left\{\begin{array}{l}
u_{n}^{i} \\
u_{s}^{i}
\end{array}\right\}=\left[\begin{array}{rr}
\cos \beta & \sin \beta \\
-\sin \beta & \cos \beta
\end{array}\right]\left\{\begin{array}{l}
u_{x}^{i} \\
u_{y}^{i}
\end{array}\right\}
$$

These constraints must be applied to all boundary nodes that have prescribed velocities.

\section{Load constraints}

To perform an upper-bound analysis, various additional constraints are imposed on the velocity field to match the type of loading. For the case shown in Fig. 17, the boundary conditions defined by equations (28) and (29) can be used to model the loading associated with the rigid footing by setting the normal velocities $w_{n}^{1}=w_{n}^{2}=-C$ along the appropriate element edges, where $C$ is some constant. The actual magnitude of $C$ does not matter, since it cancels when the loads are computed using equations (15)-(17). For a 'smooth' interface the tangential velocities $w_{s}$ underneath the footing are unrestrained, whereas for a 'rough' interface $w_{s}=0$. These types of velocity boundary conditions may be used to define the 'loading' caused by any type of stiff structure, such as a retaining wall or a pile.

For problems where part of the body is loaded by an unknown uniform normal pressure $q$, such as a flexible strip footing, it is appropriate to impose constraints on the surface normal velocities of the form

$$
\int_{A_{q}} u_{n} \mathrm{~d} A=C
$$

where $C$ is a prescribed rate of flow of material across the boundary, typically set to unity. Noting that the velocities vary linearly, substituting equation (29) into equation (30) yields the following equality constraints on the nodal velocities

$$
\frac{1}{2} \sum_{\text {edges }} L_{i j}\left[\left(u_{x}^{i}+u_{x}^{i}\right) \cos \beta_{i j}+\left(u_{y}^{i}+u_{y}^{i}\right) \sin \beta_{i j}\right]=C
$$

where $L_{i j}$ and $\beta_{i j}$ denote the length and inclination of an edge with nodes $(i, j)$, and a unit thickness is assumed. This type of constraint, when substituted into the rate of work done by the external forces, given by equation (17), permits an applied uniform pressure to be minimised directly.

Another common type of loading constraint, which is useful when a body force such as unit weight is to be optimised, takes the form

$$
\int_{V} u_{y} \mathrm{~d} A=-C
$$

where $C$ is a constant that is typically unity. This constraint permits a vertical body force to be minimised directly when the power expended by the external loads is equated to the internal power dissipation, and is particularly useful when analysing the behaviour of slopes. Noting again that the velocities vary linearly over each element, the condition in equation (31) gives rise to the following constraints on the nodal velocities

$$
\int_{V} u_{y} \mathrm{~d} A=\frac{1}{3} \sum_{\text {elements }}\left(u_{y}^{i}+u_{y}^{j}+u_{y}^{k}\right) A^{e}=-C
$$

where $(i, j, k)$ denote the nodes for some element $e$, and $A^{e}$ is the element area. 
Yield conditions

To be kinematically admissible, the stresses associated with each element (including the zero-thickness discontinuities) must satisfy the yield condition $f\left(\boldsymbol{\sigma}^{e}\right) \leqslant 0$. Since the element stresses are assumed to be constant, this requirement generates one non-linear inequality constraint for each continuum triangle and each discontinuity triangle.

\section{Upper-bound non-linear optimisation problem}

After assembling the objective function coefficients and constraints for a mesh, the upper-bound non-linear optimisation problem can be expressed as

$$
\begin{array}{ll}
\text { Minimise } & \\
\boldsymbol{\sigma}^{\mathrm{T}} \overline{\mathbf{B}} \boldsymbol{u}-\boldsymbol{c}^{\mathrm{T}} \boldsymbol{u} & \begin{array}{l}
\text { power dissipation }- \text { rate of work done } \\
\text { by fixed external forces }
\end{array}
\end{array}
$$

subject to

$\begin{array}{lll}\overline{\mathbf{B}}^{e} \boldsymbol{u}^{e} & =\dot{\alpha}^{e} \nabla f\left(\boldsymbol{\sigma}^{e}\right) & \begin{array}{l}\text { flow rule conditions for each } \\ \text { element } e\end{array} \\ \dot{\alpha}^{e} & \geqslant 0 & \begin{array}{l}\text { plastic multiplier times } A^{e} \\ \text { for each element } e\end{array} \\ \dot{\alpha}^{e} f\left(\boldsymbol{\sigma}^{e}\right)=0 & \begin{array}{l}\text { consistency condition for } \\ \text { each element } e\end{array} \\ \mathbf{A} \boldsymbol{u} & =\boldsymbol{b} & \begin{array}{l}\text { velocity boundary conditions, } \\ \text { load constraints } \\ \text { yield condition for each } \\ \text { element } e\end{array} \\ f\left(\boldsymbol{\sigma}^{e}\right) \leqslant \boldsymbol{0} & \end{array}$

where $\boldsymbol{\sigma}$ is a global vector of unknown element stresses, $\boldsymbol{u}$ is a global vector of unknown nodal velocities, $\overline{\mathbf{B}}^{e}$ is the element compatibility matrix defined by equation (12), $\overline{\mathbf{B}}=\sum \overline{\mathbf{B}}^{e}$ is a global compatibility matrix, $\boldsymbol{\sigma}^{\mathrm{T}} \overline{\mathbf{B}} \boldsymbol{u}$ is the power dissipated by plastic shearing in the continuum and discontinuities, $\boldsymbol{c}^{\mathrm{T}} \boldsymbol{u}$ is the rate of work done by fixed tractions and body forces, $\dot{\alpha}^{e}$ is the plastic multiplier times the area for element $e, f\left(\boldsymbol{\sigma}^{e}\right)$ is the yield function for element $e, \mathbf{A}$ is a matrix of equality constraint coefficients, and $\boldsymbol{b}$ is a known vector of coefficients. The solution to equation (32) constitutes a kinematically admissible velocity field, and can be found efficiently by treating the system of non-linear equations that define the Kuhn-Tucker optimality conditions. Interestingly, these optimality conditions do not involve $\dot{\alpha}^{e}$, so these quantities do not need to be included as unknowns. The two-stage quasi-Newton solver proposed by Lyamin (1999) and Lyamin \& Sloan (2002b) typically requires less than about 50 iterations, regardless of the problem size, and results in very efficient formulations for two- and threedimensional problems. This type of solver has the advantage that it can be used for general types of yield surfaces, including those with curved failure envelopes. As with the lower-bound case, however, its rate of convergence is affected by non-smooth yield criteria, and the vertices in the Tresca and Mohr-Coulomb surfaces must be smoothed to obtain good performance. Alternatively, second-order cone programming and semi-definite programming algorithms can be employed to solve equation (32) for the Tresca/Mohr-Coulomb models under two- and three-dimensional conditions respectively. As mentioned previously, both these procedures are applicable to non-smooth yield criteria, and are thus ideally suited to the Tresca and Mohr-Coulomb models. Yet another option, which is adopted in this paper, is to consider the dual of equation (32), which gives a stress-based upper-bound method (Krabbenhøft et al., 2005). The optimisation problem that results from this approach can be solved using any of the algorithms discussed above.

\section{ADAPTIVE MESH REFINEMENT}

Limit analysis is most useful when tight bounds on the collapse load are obtained. For the finite-element limit analysis methods described above, the size of the 'gap' between the bounds depends strongly on the discretisation adopted, and it is therefore desirable to investigate the possibility of developing automatic mesh refinement methods.

A comprehensive discussion of mesh generation for the lower-bound method, including stress discontinuities and 'fans' that are centred on stress singularities (such as those that occur at the edge of a rigid footing), has been given by Lyamin \& Sloan (2003). Their procedure uses a parametric mapping technique to automatically subdivide a specified number of subdomains in both two and three dimensions, and has proved invaluable for solving large-scale problems in practice. To optimise the lower bound, however, a trialand-error procedure is needed, where successively finer meshes are generated until no improvement in the limit load is found. Owing to their strong similarities to lower-bound grids, this approach can also be used to generate upperbound grids, with a similar trial-and-error approach being required.

Since a priori error estimates are not available in discrete limit analysis, a posteriori techniques are needed to predict the overall discretisation error, and hence extract a meaningful mesh refinement indicator. In one of the few studies of adaptive mesh generation for discrete limit analysis, Borges et al. (2001) presented an adaptive strategy for a mixed formulation. Their approach employed a directional error estimator, with the plastic multiplier field taken as the control variable, and permitted anisotropic mesh refinement, where elements can stretch or contract by different amounts in different directions. Computational results show that it successfully localises the elements in zones of intense plastic shearing, and significantly improves the predicted collapse loads. Although the lower-bound formulation described in the section 'Finite-element lower-bound formulation' involves only stress fields, it is possible to obtain 'quasi velocities' and 'quasi-plastic multipliers' from the dual solution to the optimisation problem described by equation (3). Exploiting this fact, Lyamin et al. (2005b) adapted the approach of Borges et al. (2001) to their lower-bound formulation, and used it to study the effects of various control variables, isotropic and anisotropic element refinement, and special 'fan' zones centred on stress singularities. They employed a modified form of the advancing-front algorithm (Peraire et al., 1987) to generate the grid, and permitted the elements to grow as well as shrink during the refinement process. The results of Lyamin et al. (2005b) show that the quasi-plastic multipliers, when used with a variety of error indicators based on recovered Hessian matrices and gradient norms, can lead to lower bounds that lie within a few per cent of the exact limit load. For problems involving strong singularities in the stress field, however, the best performance is obtained by incorporating fan zones, as these are able to model the strong rotation in the principal stresses that occurs.

More recently, exact a posteriori techniques for estimating the discretisation error in discrete limit analysis formulations have been proposed by Ciria et al. (2008) and Muñoz et al. (2009). These approaches rely on identical meshes being used for the upper- and lower-bound analyses, and provide direct measures of the contributions from each element to 
the overall bounds gap. Since the latter is precisely the quantity that needs to be minimised in practical stability calculations, this type of error estimator is innately attractive, and performs well for a wide variety of cases. In the formulations proposed by Ciria et al. (2008) and Muñoz et al. (2009), the contribution of each element to the bounds gap is found through an elaborate series of volumetric and surface integrations that include the effects of the discontinuities. For the discrete limit analysis procedures described in the sections on finite-element lower-bound and upperbound formulations these integrations are much simpler, because the discontinuities are modelled as standard continuum elements (with zero thickness), and because the upper-bound method includes stresses as unknowns as well as velocities.

To derive the element contributions to the bounds gap, the principle of virtual power is invoked for the case where identical meshes are used for the upper- and lower-bound analyses. In the upper-bound case the total plastic dissipation for the whole mesh is defined by

$$
\begin{aligned}
\int_{V} \boldsymbol{\sigma}_{\mathrm{UB}}^{\mathrm{T}} \dot{\boldsymbol{\varepsilon}}^{\mathrm{p}} \mathrm{d} V= & \int_{A_{q}} \boldsymbol{q}_{\mathrm{UB}}^{\mathrm{T}} \boldsymbol{u} \mathrm{d} A+\int_{V} \boldsymbol{h}_{\mathrm{UB}}^{\mathrm{T}} \boldsymbol{u} \mathrm{d} V \\
& +\int_{A_{t}} \boldsymbol{t}^{\mathrm{T}} \boldsymbol{u} \mathrm{d} A+\int_{V} \boldsymbol{g}^{\mathrm{T}} \boldsymbol{u} \mathrm{d} V
\end{aligned}
$$

where the subscript UB denotes upper-bound values for the unknown stresses, surface tractions and body forces. Noting that the velocities $\boldsymbol{u}$ and plastic strain rates $\dot{\boldsymbol{\varepsilon}}^{\mathrm{p}}$ are kinematically admissible throughout the domain, including the velocity discontinuities, the principle of virtual power for the computed lower-bound stresses $\boldsymbol{\sigma}_{\mathrm{LB}}$, tractions $\boldsymbol{q}_{\mathrm{LB}}$ and body forces $\boldsymbol{h}_{\mathrm{LB}}$ gives

$$
\begin{aligned}
\int_{V} \boldsymbol{\sigma}_{\mathrm{LB}}^{\mathrm{T}} \dot{\boldsymbol{\varepsilon}}^{\mathrm{p}} \mathrm{d} V= & \int_{A_{q}} \boldsymbol{q}_{\mathrm{LB}}^{\mathrm{T}} \boldsymbol{u} \mathrm{d} A+\int_{V} \boldsymbol{h}_{\mathrm{LB}}^{\mathrm{T}} \boldsymbol{u} \mathrm{d} V \\
& +\int_{A_{t}} \boldsymbol{t}^{\mathrm{T}} \boldsymbol{u} \mathrm{d} A+\int_{V} \boldsymbol{g}^{\mathrm{T}} \boldsymbol{u} \mathrm{d} V
\end{aligned}
$$

where the prescribed tractions $\boldsymbol{t}$ and body forces $\boldsymbol{h}$ are the same for each analysis. Subtracting equation (34) from equation (33) furnishes the 'dissipation gap' $\Delta$ as

$$
\begin{aligned}
\Delta & =\int_{V}\left(\boldsymbol{\sigma}_{\mathrm{UB}}-\boldsymbol{\sigma}_{\mathrm{LB}}\right)^{\mathrm{T}} \dot{\boldsymbol{\varepsilon}}^{\mathrm{p}} \mathrm{d} V \\
& =\int_{A_{q}}\left(\boldsymbol{q}_{\mathrm{UB}}-\boldsymbol{q}_{\mathrm{LB}}\right)^{\mathrm{T}} \boldsymbol{u} \mathrm{d} A+\int_{V}\left(\boldsymbol{h}_{\mathrm{UB}}-\boldsymbol{h}_{\mathrm{LB}}\right)^{\mathrm{T}} \boldsymbol{u} \mathrm{d} V
\end{aligned}
$$

For the common case of proportional loading, with the upper- and lower-bound multipliers $\left(\lambda_{\mathrm{UB}}, \lambda_{\mathrm{LB}}\right)$ defined so that $\boldsymbol{q}_{\mathrm{UB}}=\lambda_{\mathrm{UB}}^{q} \boldsymbol{q}, \boldsymbol{q}_{\mathrm{LB}}=\lambda_{\mathrm{LB}}^{q} \boldsymbol{q}, \boldsymbol{h}_{\mathrm{UB}}=\lambda_{\mathrm{UB}}^{h} \boldsymbol{h}$ and $\boldsymbol{h}_{\mathrm{LB}}=\lambda_{\mathrm{LB}}^{h} \boldsymbol{h}$, equation (35) becomes

$$
\begin{aligned}
\Delta & =\int_{V}\left(\boldsymbol{\sigma}_{\mathrm{UB}}-\boldsymbol{\sigma}_{\mathrm{LB}}\right)^{\mathrm{T}} \dot{\boldsymbol{\varepsilon}}^{\mathrm{p}} \mathrm{d} V \\
& =\left(\lambda_{\mathrm{UB}}^{q}-\lambda_{\mathrm{LB}}^{q}\right) \int_{A_{q}} \boldsymbol{q}^{\mathrm{T}} \boldsymbol{u} \mathrm{d} A+\left(\lambda_{\mathrm{UB}}^{h}-\lambda_{\mathrm{LB}}^{h}\right) \int_{V} \boldsymbol{h}^{\mathrm{T}} \boldsymbol{u} \mathrm{d} V
\end{aligned}
$$

In the above, if both the tractions and body force loads are optimised simultaneously, their corresponding multipliers must be related (e.g. by an equation such as $\lambda^{q}=\beta \lambda^{h}$, with $\beta$ being a prescribed constant). Equation (36) shows that the dissipation gap defined by

$$
\Delta=\int_{V}\left(\boldsymbol{\sigma}_{\mathrm{UB}}-\boldsymbol{\sigma}_{\mathrm{LB}}\right)^{\mathrm{T}} \dot{\boldsymbol{\varepsilon}}^{\mathrm{p}} \mathrm{d} V
$$

provides a direct measure of the difference between the upper- and lower-bound loads. Noting the usual assembly rules for a grid, it follows that

$$
\Delta=\sum_{\text {elements }} \Delta^{e}
$$

with $\Delta^{e}$ denoting the bounds gap contribution from each element. To allow for the contributions of both continuum elements and zero-thickness discontinuity elements, it is convenient to compute $\Delta^{e}$ using the relations

$$
\Delta^{e}=\left(\boldsymbol{\sigma}_{\mathrm{UB}}^{e}-\boldsymbol{\sigma}_{\mathrm{LB}}^{e}\right)^{\mathrm{T}} \overline{\mathbf{B}}^{e} \boldsymbol{u}^{e}
$$

where $\overline{\mathbf{B}}^{e}$ is the standard compatibility matrix times the element volume (defined for the two-dimensional case by equation (12)). Since the element quantities defined by equation (37) are always positive (Ciria et al., 2008), they can be used to identify elements that make large contributions to the bounds gap and are thus in need of refinement. Moreover, for the upper- and lower-bound formulations described in 'Finite-element lower-bound formulation' and 'Finiteelement upper-bound formulation', all the quantities needed to compute the error estimator are readily available, regardless of whether the element is a continuum element or a discontinuity element. As mentioned previously, the sole restriction on this type of refinement process is that identical meshes must be adopted for both the upper- and lowerbound analyses.

Using the exact error estimate provided by equation (37), the following procedure is used to adaptively refine the mesh to give tight bounds on the limit load.

1. Specify the maximum number of continuum elements allowed, $E_{\max }$, and generate an initial mesh.

2. Perform upper- and lower-bound analyses using the same mesh.

3. If the gap between the upper and lower bounds is less than a specified tolerance, or if the maximum number of continuum elements $E_{\max }$ is reached, exit with upper- and lower-bound estimates of the limit load.

4. Specify a target number of continuum elements for the current mesh iteration, $E_{i}$, with $E_{i} \leqslant E_{\max }$.

5. For each element, compute its contribution to the bounds gap $\Delta^{e}$ using equation (37). In the case of a discontinuity element, its bounds gap contribution is added to the neighbouring continuum element with which it shares the most nodes.

6. Scale the size of each continuum element to be inversely proportional to the magnitude of $\Delta^{e}$, subject to the constraint that the new number of continuum elements in the grid matches the predefined target number of continuum elements for the current iteration $E_{i}$.

7. Go to step 2 .

In the above algorithm, the target number and maximum number of continuum elements, $E_{i}$ and $E_{\max }$, are included to give the user additional control over the adaptive refinement process. In step 6 , some supplementary constraints may be included to limit the rate of decrease or increase in the element size from iteration to iteration. Typically, the maximum decrease in element size is set to a factor of 4 , and the maximum increase in size is set to a factor of 2 . These limits serve to reduce the oscillations in the size of elements as the optimum mesh is sought, and do not greatly affect the number of iterations that are needed. 
APPLICATIONS: UNDRAINED STABILITY ANALYSIS

The finite-element limit analysis formulations described above are fast and robust, and can model cases that include inhomogeneous soils, anisotropy, complex loading, natural discontinuities, complicated boundary conditions, and three dimensions. They not only give the limit load directly, without the need for an incremental analysis, but also bracket the solution from above and below, thereby giving an exact estimate of the mesh discretisation error. These features greatly enhance the practical utility of the bounding theorems, especially in three dimensions, where conventional incremental methods are often expensive and difficult to use. In this section, the finite-element limit analysis methods are used to study a variety of undrained stability problems. The results will serve to illustrate the types of case that can be tackled, and the quality of the solutions that can be obtained.

\section{Bearing capacity of rigid strip footing on clay with heterogeneous strength}

First a rigid footing is considered, of width $B$, resting on clay that has an undrained surface shear strength of $s_{\mathrm{u} 0}$ and a rate of strength increase with depth equal to $\rho$, as shown in Fig. 21. Following Davis \& Booker (1973), the bearing capacity can be expressed in the form

$$
\frac{Q_{\mathrm{u}}}{B}=F\left[(2+\pi) s_{\mathrm{u} 0}+\frac{\rho B}{4}\right]
$$

where $F$ is a factor that depends on the dimensionless quantity $\rho B / s_{\mathrm{u} 0}$ and the footing roughness.

Figure 22 compares the bearing capacity factors from finite-element limit analysis, using adaptive mesh refinement

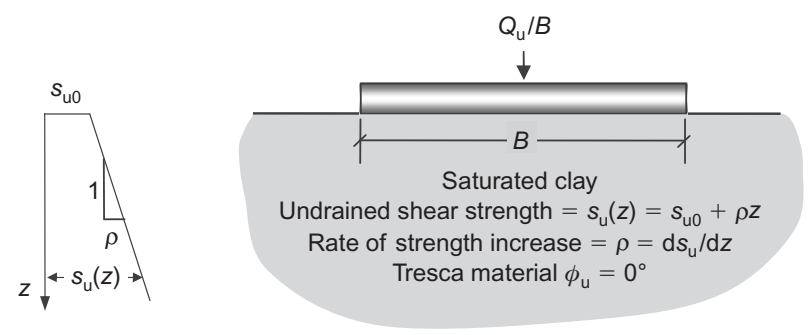

Fig. 21. Rigid footing on clay whose undrained strength increases with depth

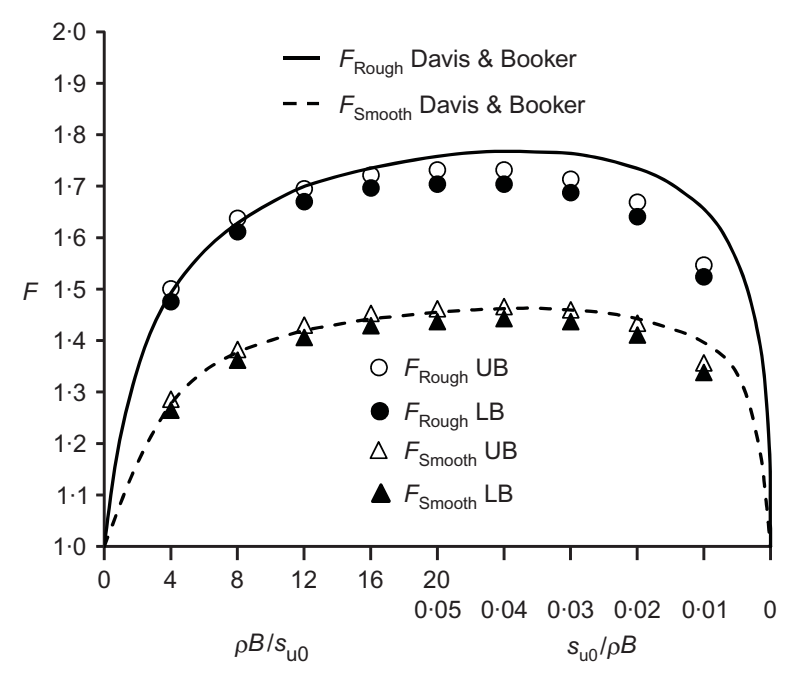

Fig. 22. Bearing capacities predicted by finite-element limit analysis and method of characteristics (Davis \& Booker, 1973) with a maximum of 4000 continuum elements, with the solutions of Davis \& Booker (1973) obtained using the method of characteristics. Results are presented for both smooth and rough footings, and the split scale on the horizontal axis accounts for the two limiting cases of uniform shear strength $\left(\rho B / s_{\mathrm{u} 0}=0\right)$ and zero surface strength $\left(s_{\mathrm{u} 0} / \rho B=0\right)$. Whereas there is close agreement between the limit analysis and characteristics solutions for $\rho B / s_{\mathrm{u} 0} \leqslant 8$, significant differences are evident for low values of $s_{\mathrm{u} 0} / \rho B$ where the surface shear strength is small. To resolve this surprising inconsistency, the problem was reanalysed using the stress characteristics program $\mathrm{ABC}$, developed at Oxford by Martin (2004). This program provides a partial lowerbound stress field, and adaptively refines the mesh of characteristics to ensure the solution is accurate. In a private communication, Martin (personal communication, 2011) confirmed that, for each of the cases considered, the lowerbound stress field from $\mathrm{ABC}$ can be extended throughout the soil mass without violating equilibrium or yield, and that it can also be associated with a velocity field that gives a coincident upper-bound collapse load. This suggests that the solutions from $A B C$, shown in Fig. 23, are exact estimates of the bearing capacity. Indeed, there is excellent agreement between these characteristics solutions and the new finiteelement limit analysis solutions, with a maximum difference of less than $1 \%$.

When the quantity $s_{\mathrm{u} 0} / \rho B$ is small, the failure mechanism involves soil being squeezed out in a thin band underneath the footing. Unless adaptive meshing is employed for these cases, such as that used in the program $\mathrm{ABC}$, the method of characteristics will be unable to model the true failure mechanism with high accuracy. This explains the discrepancies observed with the solutions of Davis \& Booker (1973). The finite-element limit analysis methods have no difficulty in dealing with this extreme case, since the adaptive meshing strategy, with the bounds gap error estimator, automatically concentrates the elements where they are needed.

Figure 24 further highlights the difficulties that can arise when numerical methods are used to predict the limit load associated with a highly localised failure mechanism. The plot shows the ratio of the bearing capacity found from the limit-equilibrium analysis of Raymond (1967) to the bearing capacity found from finite-element limit analysis (taken as the average of the upper and lower bounds, which are within $1 \%$ of the characteristics solutions of Martin (personal communication, 2011). Except for the case of uniform strength $\left(\rho B / s_{\mathrm{u} 0}=0\right)$, the limit-equilibrium method, which

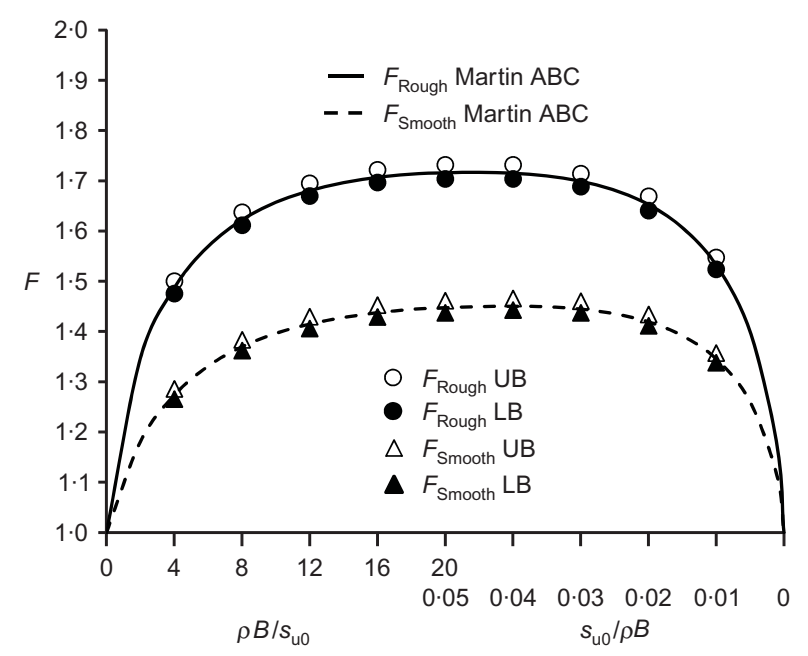

Fig. 23. Bearing capacities predicted by finite-element limit analysis and method of characteristics (Martin, personal communication, 2011) 


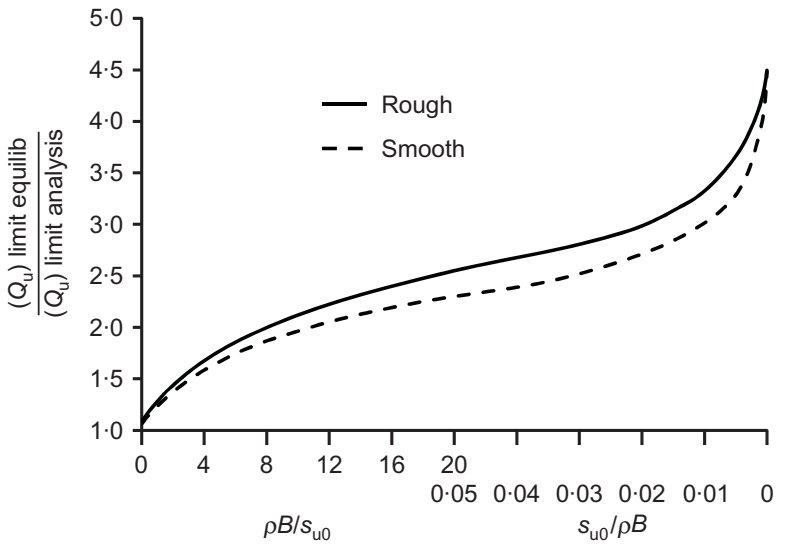

Fig. 24. Bearing capacities predicted by finite-element limit analysis and limit equilibrium (Raymond, 1967)

assumes a circular slip surface, furnishes solutions that are typically two to three times greater than the exact values. For the worst case, where the surface shear strength is zero and the failure mechanism is highly localised, the limitequilibrium solution overestimates the exact bearing capacity by a factor of approximately $4 \cdot 5$.

The large error in the bearing capacity predictions indicated in Fig. 24 is due to the inability of a circular slip surface to model the actual mode of failure, especially for cases where $s_{\mathrm{u} 0} / \rho B$ is small. This is shown clearly in Fig. 25 , which compares the failure surfaces predicted by limit equilibrium with the failure mechanisms (contours of plastic dissipation) predicted from adaptive upper-bound limit analyses for a rough footing on two soils with $\rho B / s_{\mathrm{u} 0}=4$ and $\rho B / s_{\mathrm{u} 0}=100$. For the latter case, the zones of plastic deformation at collapse are highly localised, and occur in close proximity to the underside of the footing. The limitequilibrium method, because it assumes a circular failure surface, is unable to replicate this mode of deformation, and thus overpredicts the bearing capacity. Contours of plastic dissipation, like those shown in Fig. 25, provide a clear indication of zones of intense plastic shearing, and are useful tools for visualising collapse mechanisms when using finite-element limit analysis to solve practical stability problems in geotechnical engineering.

The meshes generated by the adaptive mesh refinement scheme, using the bounds gap error indicator described in the section 'Adaptive mesh refinement' with a maximum limit of 2000 elements, are shown in Fig. 26 for the case $\rho B / s_{\mathrm{u} 0}=4$. These plots show that the adaptive scheme converges to the optimum mesh after four cycles of refinement, and gives bounds that bracket the exact solutions to within 1\%. Subsequent mesh refinement cycles do not improve the estimate of the bearing capacity, owing to the restriction of using 2000 elements, and more accurate predictions would require this limit to be increased. Note that extension elements were used to check the completeness of the lower-bound stress field for the finest mesh, but these have been omitted from the plot for clarity. The bounds gap error indicator clearly concentrates the elements in the zones of intense plastic shearing that are shown in Fig. 25. Computationally, the upper- and lower-bound limit analysis methods are very fast, with each solution requiring around $2 \mathrm{~s}$ of CPU time on a standard desktop machine for a grid with 2000 elements.

\section{Strip footing under inclined eccentric loading}

Now the problem, defined in Fig. 27, of a rigid strip footing, subject to an inclined eccentric load, resting on a soil with uniform undrained shear strength $s_{\mathrm{u}}$ is considered. To predict the magnitude of the load $P$, the influence of three different footing interface models is examined.

(a) Tension permitted with a shear capacity equal to the undrained strength.

(b) No tension permitted, but no limit on the shear capacity.

(c) No tension permitted, with a shear capacity equal to the undrained strength.

The first of these models is often assumed in practice because of its simplicity, while the third model provides a better representation of actual interface behaviour.

The optimised meshes and associated failure mechanisms for the three cases are shown in Fig. 28. Using the average of the upper and lower bounds to estimate the collapse load, and a maximum number of elements equal to 3000 , the exact solutions are bracketed to within $\pm 1.6 \%$ for the various interface conditions. As expected, the flow rule for case (a) ensures that no interface separation occurs, while the flow rule for case (b) dictates that no relative shear deformation arises, with all motion being normal to the interface. Owing to the effect of the no-tension constraint, case (c), which is a reasonable approximation to the "no suction' conditions that might apply in practice, gives an average collapse load that is approximately $19 \%$ lower than that for case (a). The power dissipation plots in Fig. 28 highlight the different collapse mechanisms that occur for the three cases, with case (b) exhibiting an interesting double failure surface. These examples underscore the versa-

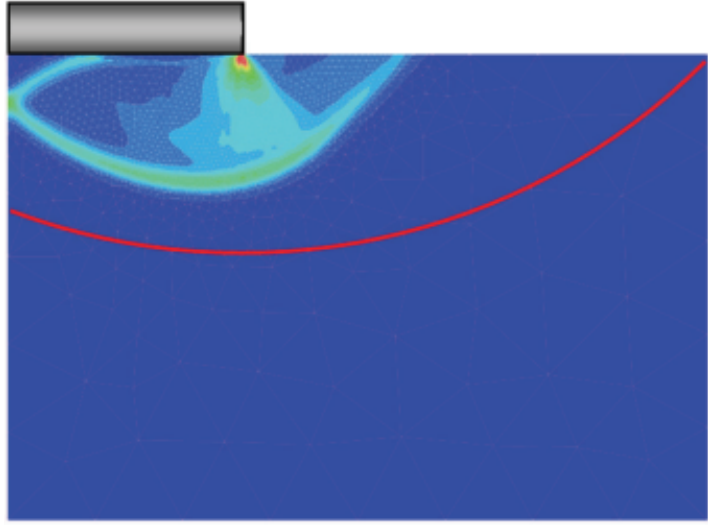

$\rho B / s_{\mathrm{u} 0}=4$

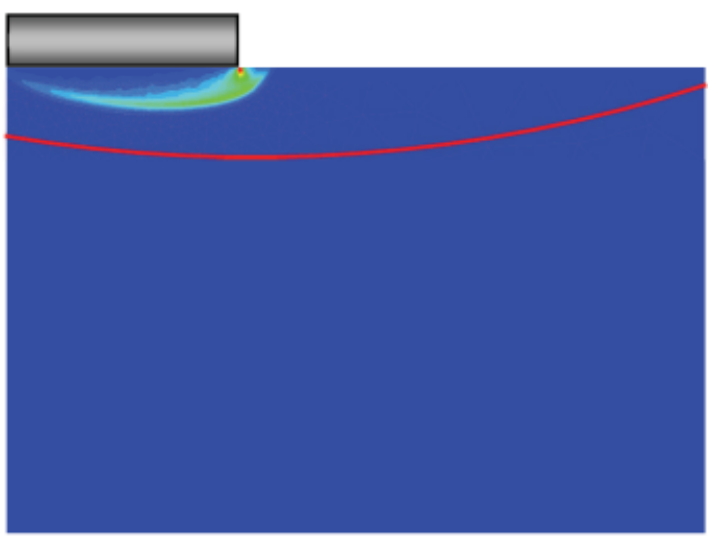

$\rho B / s_{\mathrm{u} 0}=100$

Fig. 25. Failure mechanisms predicted by upper-bound limit analysis and limit equilibrium (Raymond, 1967) 

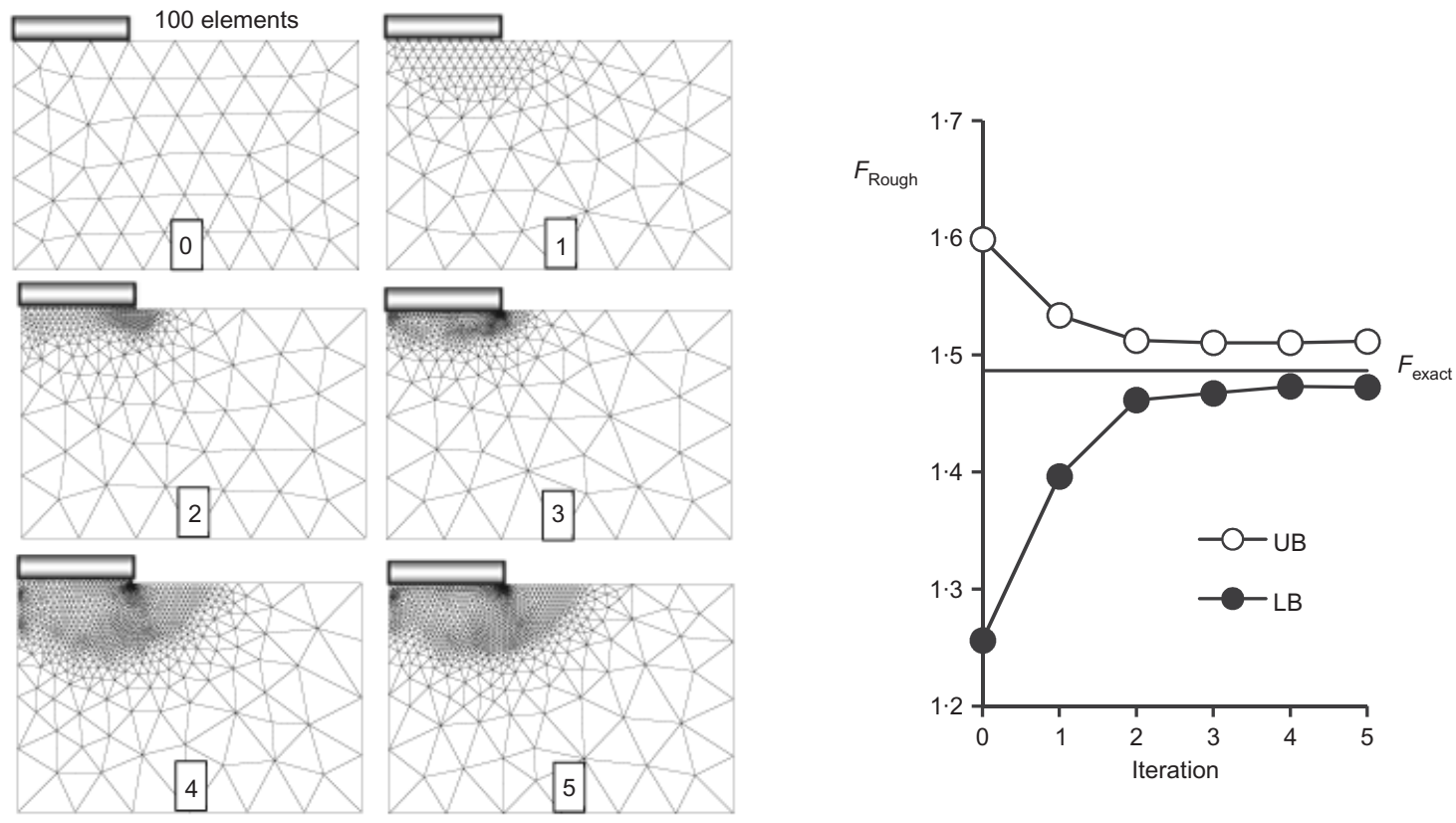

2000 elements

Fig. 26. Adaptive mesh refinement for finite-element limit analysis

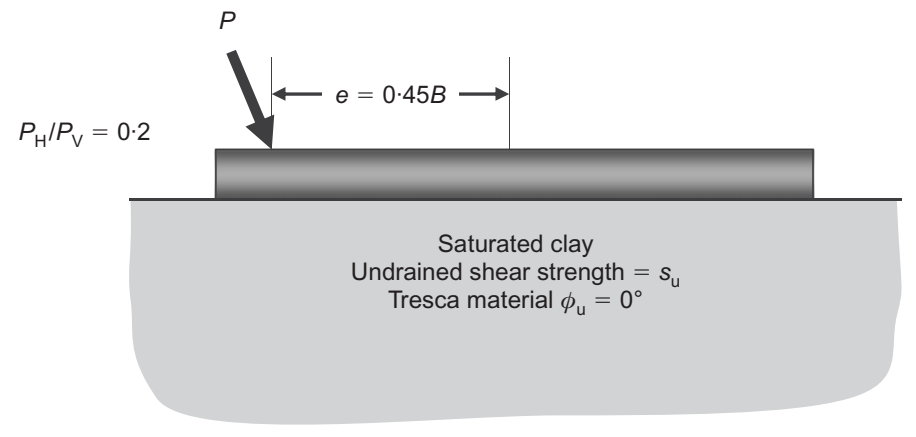

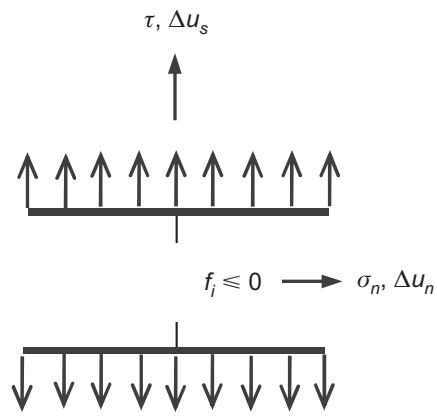

Case (a)

Tension allowed

$\left|\tau_{\max }\right| \leqslant s_{\mathrm{u}}$

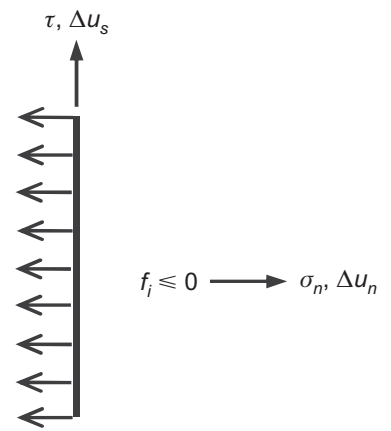

Case (b)

No tension allowed

No limit on $\tau_{\max }$

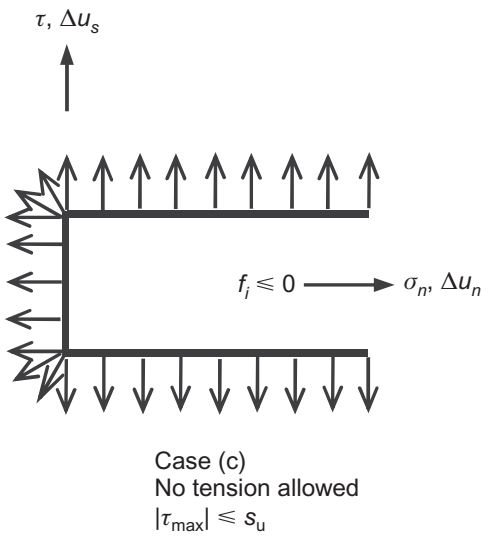

Fig. 27. Rigid footing subject to an inclined eccentric load

tility of the finite-element limit analysis formulations in modelling complex interface conditions, as well as the benefits of adaptive mesh generation.

In closing, it is noted that a detailed study of the behaviour of strip footings under combined vertical, horizontal and moment (V, H, M) loading can be found in Ukritchon et al. (1998). Using modified versions of the early finiteelement limit analysis programs developed by Sloan (1988a) and Sloan \& Kleeman (1995), they derive comprehensive three-dimensional failure envelopes that account for the effects of underbase suction and heterogeneous undrained strength profiles. These envelopes suggest that the traditional empirical bearing capacity factors for inclined eccentric loading are conservative, often underestimating the exact values by more than $25 \%$. Moreover, for problems where there is a significant strength gradient, these empirical factors are unreliable, and not recommended for practical use.

\section{Stability of plane-strain tunnel and tunnel heading}

The undrained stability of a circular tunnel in clay, whose shear strength increases linearly with depth, has been studied by several researchers, including Davis et al. (1980), Sloan 

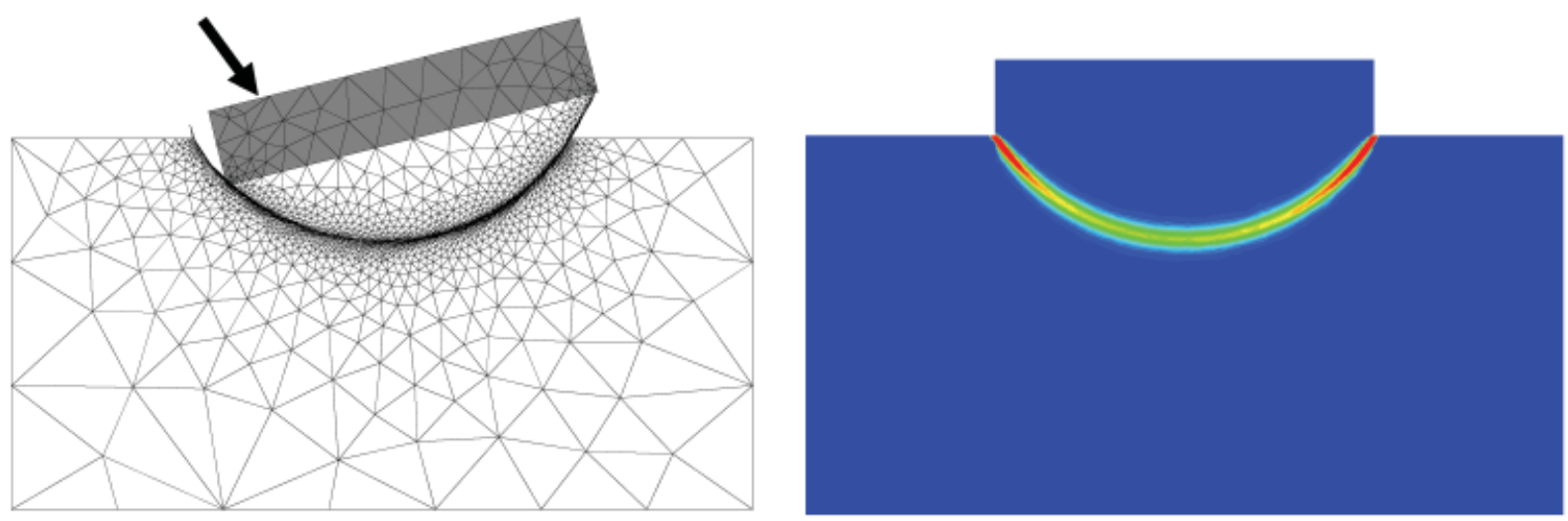

Case (a): $P_{\mathrm{LB}}=1 \cdot 78 B s_{\mathrm{u}}, P_{\mathrm{UB}}=1 \cdot 82 B s_{\mathrm{u}}$
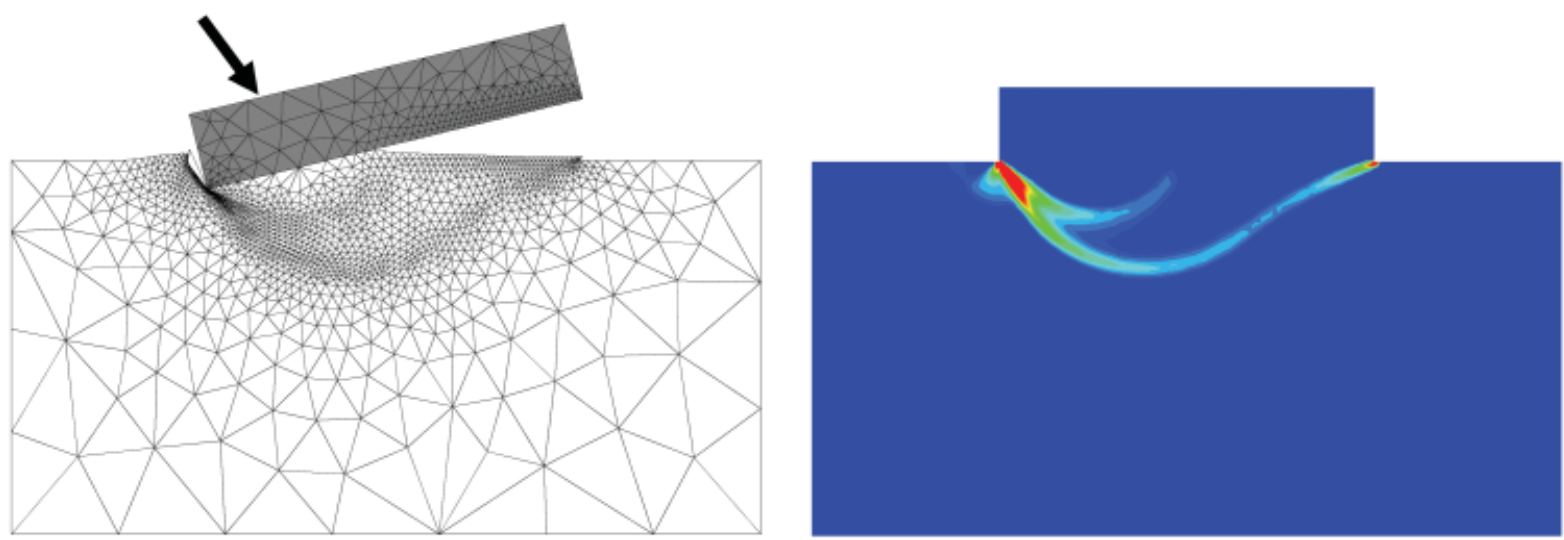

Case (b): $P_{\mathrm{LB}}=1.53 B s_{\mathrm{u}}, P_{\mathrm{UB}}=1.58 B s_{\mathrm{u}}$
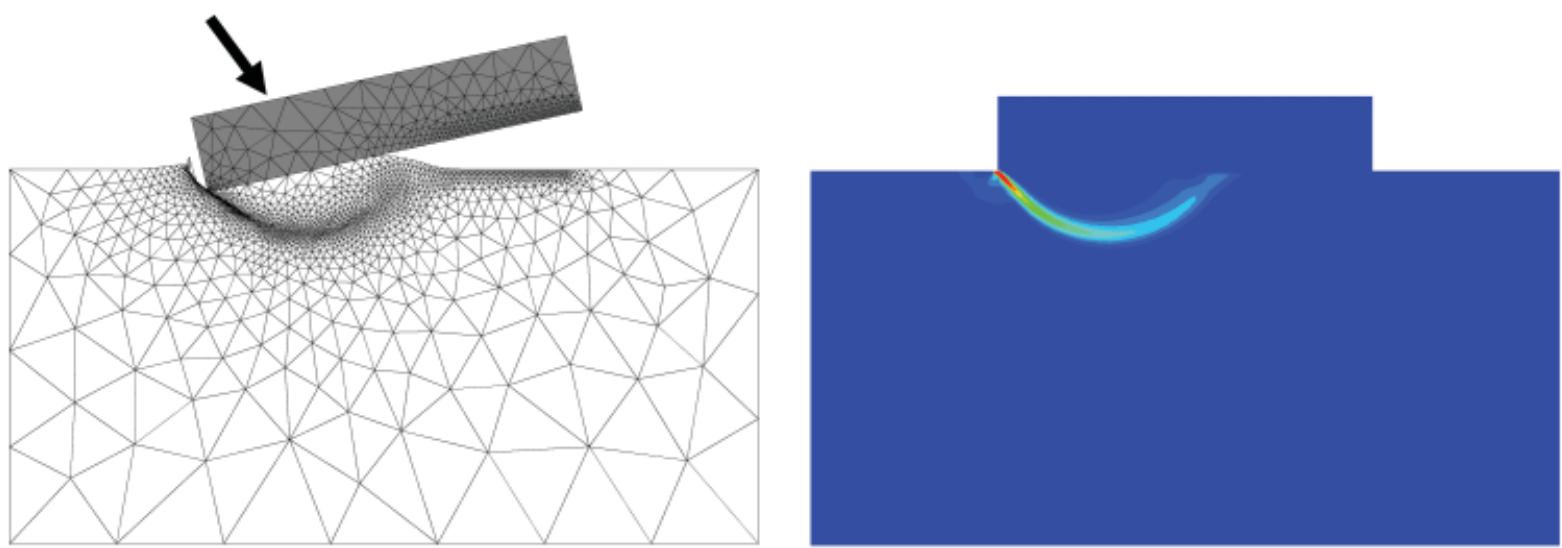

Case (c): $P_{\mathrm{LB}}=1 \cdot 45 B s_{\mathrm{u}}, P_{\mathrm{UB}}=1 \cdot 48 B s_{\mathrm{u}}$

Fig. 28. Meshes and failure mechanisms for rigid footing subject to an inclined eccentric load

\& Assadi (1992) and Wilson et al. (2011). The problem is defined in Fig. 29, where a tunnel of diameter $D$ and cover $C$ is embedded in a soil with a surface undrained strength $s_{\mathrm{u} 0}$ and a strength gradient with depth $\rho$. This idealised case models a bored tunnel in soft ground where a rigid lining is inserted as the excavation proceeds, and the unlined heading, of length $P$, is supported by an internal pressure $\sigma_{\mathrm{t}}$. Collapse of the heading is driven by the action of the surcharge $\sigma_{\mathrm{s}}$ and the soil unit weight $\gamma$. The assumption of plane strain is clearly valid only when $P \gg D$, but the stability for this case is more critical than that of a three-dimensional tunnel heading, and thus it yields a conservative estimate of the loads needed to trigger collapse. For the purposes of analysis, it is convenient to describe the stability of the tunnel by two dimensionless load parameters, $\left(\sigma_{\mathrm{s}}-\sigma_{\mathrm{t}}\right) / s_{\mathrm{u} 0}$ and $\gamma D / s_{\mathrm{u} 0}$. In practice, the unlined heading is typically supported by either compressed air or clay slurry as the tunnel is excavated, and the known quantities are $C / D, P / D, \gamma D / s_{\mathrm{u} 0}$ and $\rho D / s_{\mathrm{u} 0}$, with the value of $\left(\sigma_{\mathrm{s}}-\sigma_{\mathrm{t}}\right) / s_{\mathrm{u} 0}$ at incipient collapse being unknown.

Before tackling the stability of a three-dimensional tunnel heading, first the plane-strain problem shown as section $\mathrm{A}-\mathrm{A}$ is considered. For this case, $P / D$ may be omitted from the analysis, and the relevant stability parameter is

$$
\frac{\sigma_{\mathrm{s}}-\sigma_{\mathrm{t}}}{s_{\mathrm{u} 0}}=f\left(\frac{C}{D}, \frac{\gamma D}{s_{\mathrm{u} 0}}, \frac{\rho D}{s_{\mathrm{u} 0}}\right)
$$

To analyse this problem, the quantities $s_{\mathrm{u} 0}, \rho, \gamma, H, D$ and $\sigma_{\mathrm{s}}$ are fixed, and the value of $-\sigma_{\mathrm{t}}$ (i.e. the tensile stress on the face of the tunnel) is optimised. Alternatively, it is possible to fix the value of $\sigma_{\mathrm{t}}$ and optimise the surcharge $\sigma_{\mathrm{s}}$. 

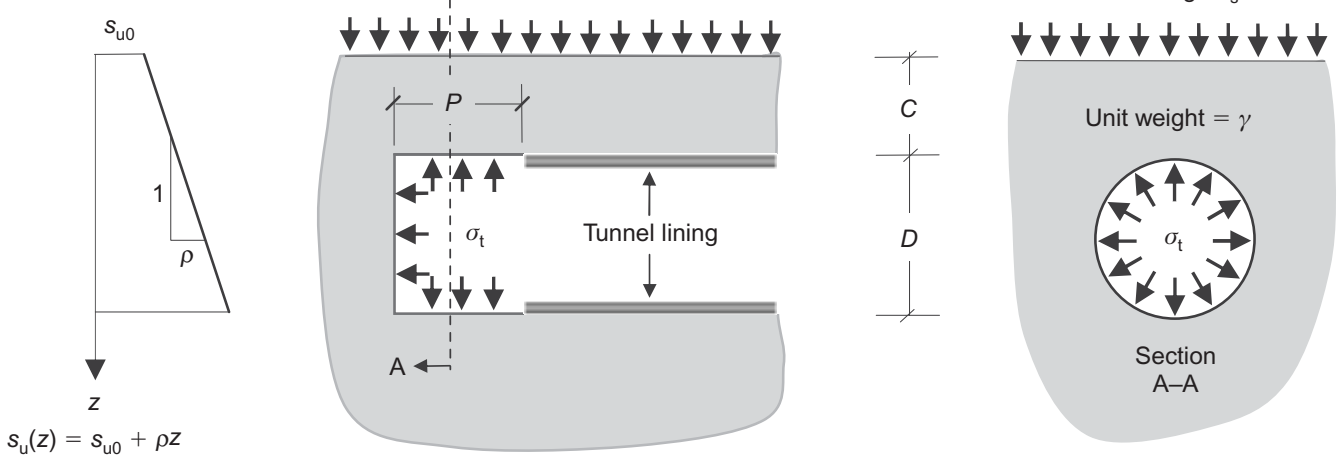

Stability number $\left(\sigma_{\mathrm{s}}-\sigma_{\mathrm{t}}\right) / s_{\mathrm{u} 0}=f\left(C / D, P / D, \gamma D / s_{\mathrm{u} 0}, \rho D / s_{\mathrm{u} 0}\right)$

Fig. 29. Stability of circular tunnel in undrained clay

Figure 30 shows upper and lower bounds on the stability parameter $\left(\sigma_{\mathrm{s}}-\sigma_{\mathrm{t}}\right) / s_{\mathrm{u} 0}$, plotted as a function of the dimensionless unit weight $\gamma D / s_{\mathrm{u} 0}$ and the soil strength factor $\rho D /$ $s_{\mathrm{u} 0}$, for two tunnels with cover-to-diameter ratios of $C / D=4$ and $C / D=10$. These bounds bracket the exact stability parameter to within a few per cent, and were found from adaptive finite-element limit analysis using a maximum of around 4000 elements.

By definition, a negative value of $N=\left(\sigma_{\mathrm{s}}-\sigma_{\mathrm{t}}\right) / s_{\mathrm{u} 0}$ indicates that a compressive normal stress of at least $\left|\sigma_{\mathrm{s}}-N s_{\mathrm{u} 0}\right|$ must be applied to the tunnel wall to support the imposed loads, whereas a positive value of $N$ implies that no internal tunnel support is required to maintain stability provided $\sigma_{\mathrm{s}} \leqslant N s_{\mathrm{u} 0}$. Indeed, in the latter case, the tunnel is theoretically capable of sustaining a uniform tensile pressure up to $\left|\sigma_{\mathrm{s}}-N s_{\mathrm{u} 0}\right|$ without undergoing collapse. Points that lie on the horizontal axis defined by $\sigma_{\mathrm{s}}-\sigma_{\mathrm{t}}=0$ indicate configurations for which the tunnel pressure must precisely balance the ground surcharge in order to prevent collapse.

Figure 31 shows the optimised limit analysis mesh and

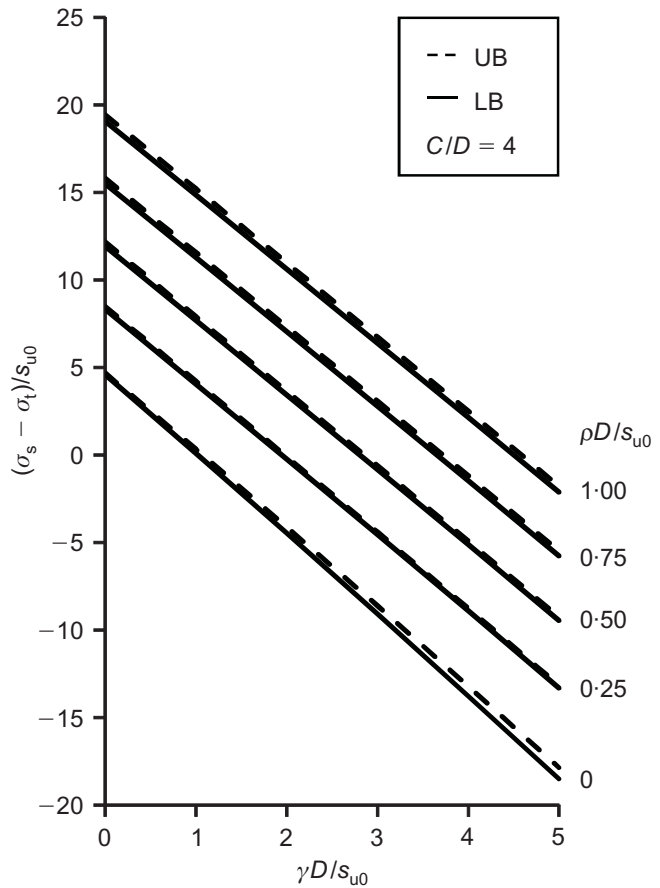

(a) power dissipation plot for a tunnel with $C / D=4$ in a soil with $\gamma D / s_{\mathrm{u} 0}=3$ and a uniform strength profile. In the lowerbound analyses, extension elements were added around the border of the grid (not shown) to propagate the statically admissible stress field over the semi-infinite domain. This step has a negligible effect on the computed stability parameter, but ensures that the lower-bound results are truly rigorous. For the finest grid, with around 4000 elements, each bound calculation required about $4 \mathrm{~s}$ of CPU time on a desktop machine, and the optimum arrangement was deduced after four cycles of refinement. The ability of the adaptive mesh refinement scheme to concentrate the elements where they are needed is again apparent. Fig. 32 shows the failure mechanism for the same example, but with a finite strength gradient. Compared with the case with uniform strength, shown in Fig. 31, the zone of plastic deformation is much more localised, and, as expected, does not extend below the invert, where the strength is higher.

The predictions from limit analysis theory are compared with the centrifuge results of Mair (1979) in Fig. 33. For the

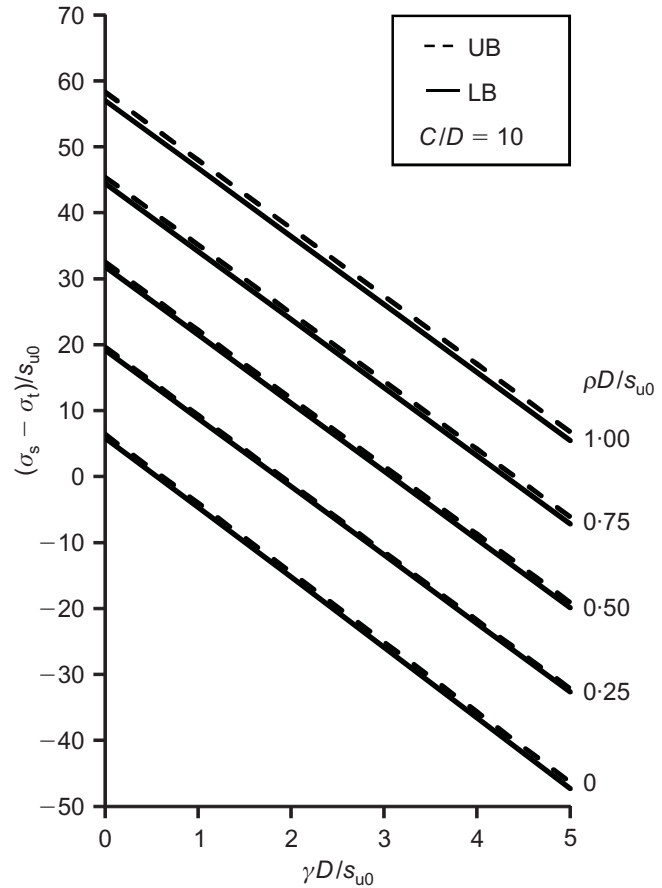

(b)

Fig. 30. Stability bounds for plane-strain tunnel: (a) $C / D=4$; (b) $C / D=10$ 

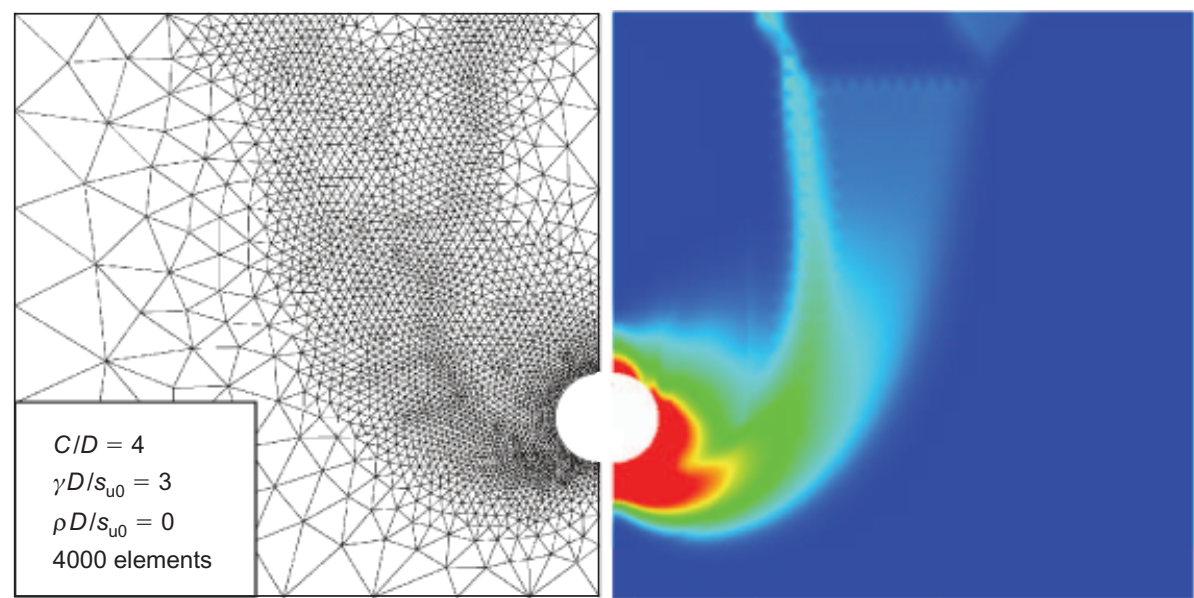

Fig. 31. Optimised mesh and failure mechanism for circular tunnel with $C / D=4$ and uniform strength

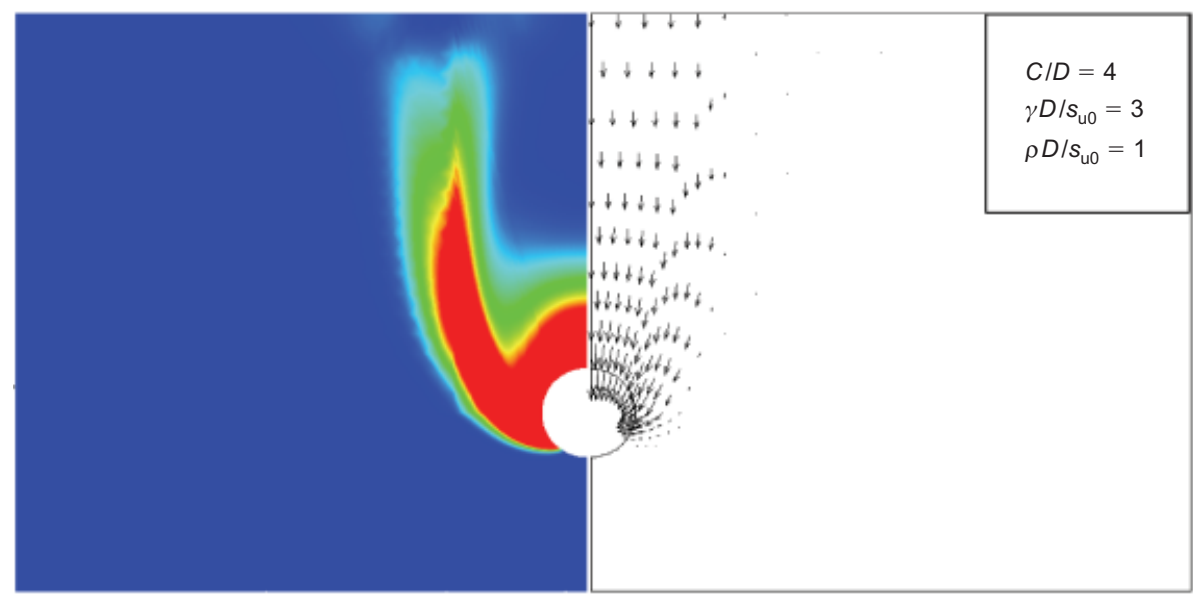

Fig. 32. Failure mechanism for circular tunnel with $C / D=4$ and finite strength gradient

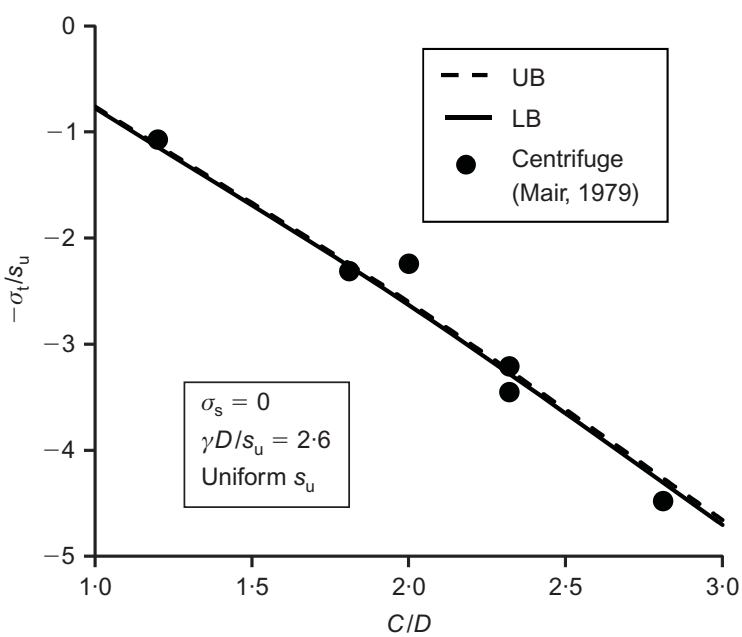

Fig. 33. Comparison of limit analysis predictions with centrifuge results for plane-strain tunnel

case of a plane-strain tunnel in kaolin clay with a uniform strength profile and zero surcharge, the stability bounds predicted by limit analysis are in excellent agreement with the experimental observations.

Finite-element limit analysis results for a three-dimensional tunnel heading in kaolin clay with a uniform strength profile are shown in Fig. 34. These curves indicate the tunnel pressure required to maintain stability, $\sigma_{\mathrm{t}} / s_{\mathrm{u}}$, as a function of the unsupported heading length $P / D$ for a case where $C / D=3$ and $\gamma D / s_{\mathrm{u}}=3 \cdot 6$. Note that, since the tunnel support pressure works against failure in this instance, an upper-bound calculation actually gives a lower bound on the support pressure, and vice versa. Fig. 34 also shows the theoretical collapse pressure (accurate to 1\%) for the corresponding case of a plane-strain tunnel where $P / D \rightarrow \infty$, as

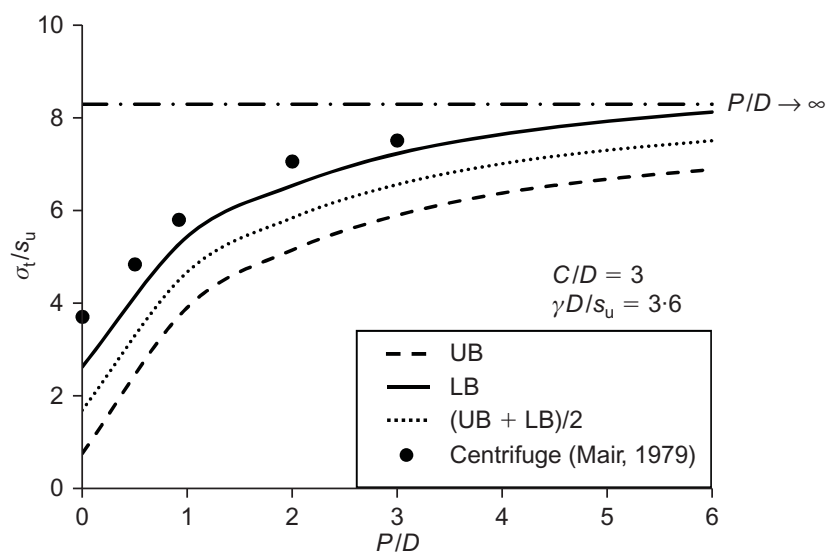

Fig. 34. Comparison of limit analysis predictions with centrifuge results for three-dimensional tunnel heading 
well as the centrifuge results taken from Mair (1979). In this example there is a bigger gap between the upper and lower bounds, since a trial-and-error meshing procedure was necessary using 20000-30000 tetrahedral elements (the threedimensional adaptive limit analysis methods are still under development). Overall, however, the lower-bound tunnel pressure predictions are close to the observed results of Mair (1979), and asymptote clearly towards the limiting value for plane-strain conditions. For values of $P / D \geqslant 1$, the average of the upper and lower bounds underpredicts the measured tunnel pressures by a maximum of $15 \%$. Not surprisingly, this problem is computationally demanding, and typically required around $4 \mathrm{~h}$ of CPU time for each analysis with a grid of 30000 tetrahedral elements.

Figure 35 shows the power dissipation plots for two of the cases in Fig. 34, where $P / D=0$ and $P / D=4$. In the former, the failure mechanism involves mostly soil that is directly above or in front of the tunnel face, and comprises several zones of intense plastic shearing. As expected, failure in the case of $P / D=4$ is associated with a much larger zone of plastic deformation, including collapse of the tunnel roof and heave of the tunnel floor. Clearly, however, the mode of plastic deformation is not uniform along the length of the tunnel, which suggests that the condition of plane strain has not been reached. For all the lower-bound analyses, threedimensional extension elements were added to the edge of the grids (not shown in Fig. 35) to ensure that the stress fields were statically admissible over the semi-infinite domain.

\section{CONSEQUENCES OF AN ASSOCIATED FLOW RULE}

For total stress analysis of the undrained stability of clays, where the friction angle is assumed to be zero and all deformation takes place at constant volume, the assumption of an associated flow rule has little influence on the failure load. For drained stability analysis involving soils with high friction angles, however, the use of an associated flow rule predicts excessive dilation during shear failure, and raises the question of whether the bound theorems will provide realistic estimates of the limit load.

\section{Theorems for non-associated flow rules}

In a pioneering investigation of the crucial issue of nonassociated flow, Davis (1968) argued that the flow rule will not have a major influence on the limit load for frictional soils unless the problem is strongly constrained in a kinematic sense. A precise definition of the degree of kinematic constraint is elusive, but many geotechnical collapse modes are not strongly constrained, since they involve a freely deforming ground surface and a semi-infinite domain. For these cases, Davis (1968) conjectured that it is reasonable to assume that the bound theorems will give acceptable estimates of the true limit load. In addition, by examining the failure behaviour on slip-lines for a non-associated MohrCoulomb material, he established that the shear and normal stress are related by

$$
\tau=\sigma_{n} \tan \phi^{*}+c^{*}
$$

where $c^{*}$ and $\phi^{*}$ are 'reduced' strength parameters, defined by

$$
\left.\begin{array}{l}
c^{*}=\beta c^{\prime} \\
\tan \phi^{*}=\beta \tan \phi^{\prime}
\end{array}\right\} \beta=\frac{\cos \psi^{\prime} \cos \phi^{\prime}}{1-\sin \psi^{\prime} \sin \phi^{\prime}}
$$

and $c^{\prime}$ is the effective cohesion, $\phi^{\prime}$ is the effective friction angle and $\psi^{\prime}$ is the dilation angle. The use of these reduced strengths provides a practical means for dealing with nonassociated flow in limit analysis, and will be explored later in this paper. When considering the behaviour of real soil it should, of course, be remembered that the dilation angle actually varies during the plastic deformation that precedes failure, and in fact approaches zero at the critical state. Nonetheless, in the absence of laboratory or field data, a constant rate of dilation is often assumed in practice, with values in the range $0 \leqslant \psi^{\prime} \leqslant \phi^{\prime} / 3$ being typical.

Apart from the approach suggested above by Davis (1968), very few useful theoretical results are available for modelling non-associated flow in a cohesive-frictional soil. If a plastic potential $g$ is defined so that the plastic strain rates are now given by $\dot{\varepsilon}_{i j}^{\mathrm{p}}=\dot{\lambda} \partial g / \partial \sigma_{i j}$, where $g$ is convex and contained within the yield surface $f$, the impact of the flow rule can be estimated by using the following results.

(a) A conventional upper-bound calculation gives a rigorous upper bound on the limit load for an equivalent material with a non-associated flow rule (Davis, 1968).

(b) A rigorous lower bound on the limit load for a nonassociated material can be obtained by substituting the plastic potential for the yield criterion in the static admissibility conditions (Palmer, 1966).

Although conceptually valuable, these two theorems frequently furnish weak bounds if the dilation angle is consider-

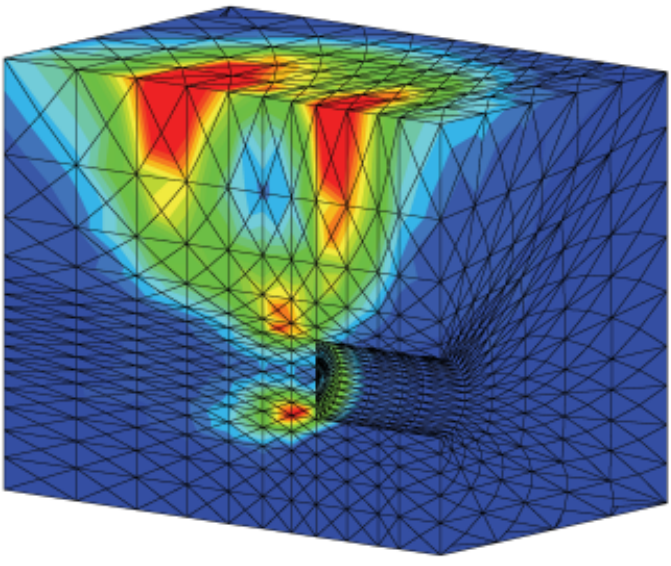

$P / D=0$

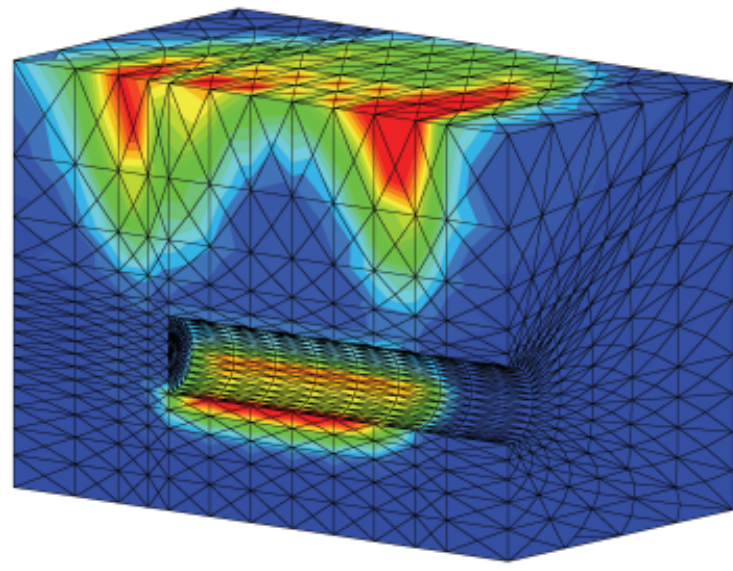

$P / D=4$

Fig. 35. Failure mechanisms for three-dimensional tunnel headings with $C / D=3$ and $\gamma D / s_{\mathrm{u}}=3 \cdot 6$ 
ably less than the friction angle. Unfortunately, this is often the case for materials with high friction angles, such as dense sands. Drescher \& Detournay (1993), in a stronger result, proved that the limit load obtained from a rigid block mechanism with Davis' discontinuity strengths $c^{*}$ and $\phi^{*}$, as defined in equations (38), gives an upper bound on the true limit load for a non-associated material with parameters $\left(c^{\prime}, \phi^{\prime}, \psi^{\prime}\right)$. This theorem suggests that limit analysis with Davis' reduced strength parameters may provide useful estimates of the limit load, provided collapse is triggered by localised plastic deformation along a well-defined failure surface.

\section{Volume change behaviour of real soil}

For a Mohr-Coulomb material undergoing plastic deformation, the shear strength is governed by the effective cohesion $c^{\prime}$ and friction angle $\phi^{\prime}$, while the volume change is controlled by the dilation angle $\psi^{\prime}$. With an associated flow rule it is assumed implicitly that $\psi^{\prime}=\phi^{\prime}$, whereas for a real soil $\psi^{\prime}<\phi^{\prime}$, so that plastic deformation obeys a nonassociated flow rule. Fig. 36 shows the dilation predicted by these two assumptions for plastic shearing along a planar failure surface. For the same shear displacement increment (velocity jump) $\Delta u_{s}$, the associated flow rule gives a larger normal displacement increment (velocity jump) $\Delta u_{n}$, and hence a larger volume change in the material.

Under a general state of stress, the volumetric plastic strain rate is related to the maximum principal strain rate by $\dot{\varepsilon}_{\mathrm{v}}^{\mathrm{p}}=\left[\tan ^{2}\left(45^{\circ}+\psi^{\prime} / 2\right)-1\right] \dot{\varepsilon}_{1}^{\mathrm{p}}$, where tensile strains are taken as positive. Typical plots of $\dot{\varepsilon}_{\mathrm{v}}^{\mathrm{p}}$ against $\dot{\varepsilon}_{1}^{\mathrm{p}}$ for a variety of soils, shown in Fig. 37, indicate clearly that the dilation angle varies throughout the process of failure, and eventually approaches zero at the critical state. Moreover, even in stress ranges where the rate of volume change is constant, the dilation angle is often appreciably less than the corresponding friction angle. All the above observations suggest that great care should be exercised when using a simple MohrCoulomb model with an associated flow rule to predict the limit load under drained loading conditions, particularly for soils with high friction angles.

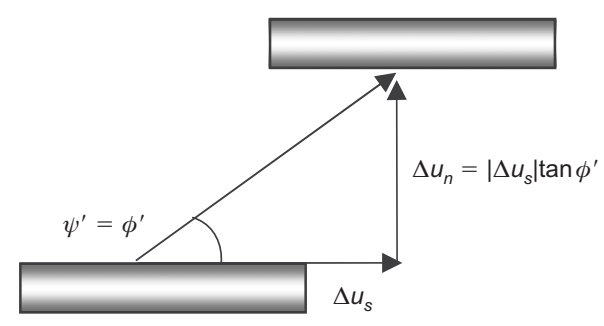

Associated flow rule

\section{Biaxial test with Mohr-Coulomb material}

To further investigate the influence of the flow rule on the collapse load for a cohesive-frictional problem, the biaxial compression of a plane-strain block of Mohr-Coulomb material is now considered, as shown in Fig. 38. Two lengthto-width ratios of $L / B=1$ and $L / B=3$ are analysed, each using a rigidity index $G / c^{\prime}=300$ and Mohr-Coulomb parameters of $\phi^{\prime}=30^{\circ}$ and $\psi^{\prime}=0^{\circ}, 15^{\circ}, \phi^{\prime}$. Provided the sample length is such that $L \geqslant B \tan \left(45^{\circ}+\psi^{\prime} / 2\right)$, a failure plane is free to form across the specimen at an angle of $\theta=45^{\circ}+\psi^{\prime} / 2$ to the horizontal, and the exact collapse pressure is given by $q_{\mathrm{u}}=2 c^{\prime} \tan \left(45^{\circ}+\phi^{\prime} / 2\right)$. For shorter samples where $L<B \tan \left(45^{\circ}+\psi^{\prime} / 2\right)$, the exact collapse pressure is unknown and must be determined numerically.

To begin the investigation, the displacement finite-element computer program SNAC (Abbo \& Sloan, 2000) was used to analyse this problem with both associated and nonassociated flow rules. The mesh employed for $L / B=1$ is shown in Fig. 39, and comprises 800 quartic triangles. A similar mesh is used for the case $L / B=3$, except that the

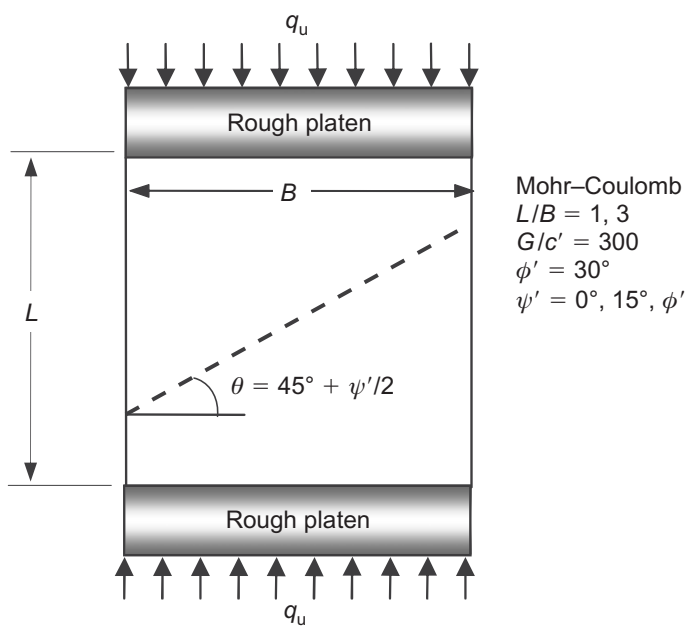

Fig. 38. Biaxial compression of Mohr-Coulomb block

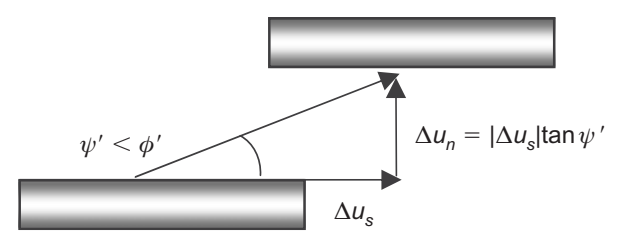

Non-associated flow rule

Fig. 36. Dilation during shearing on a planar failure surface
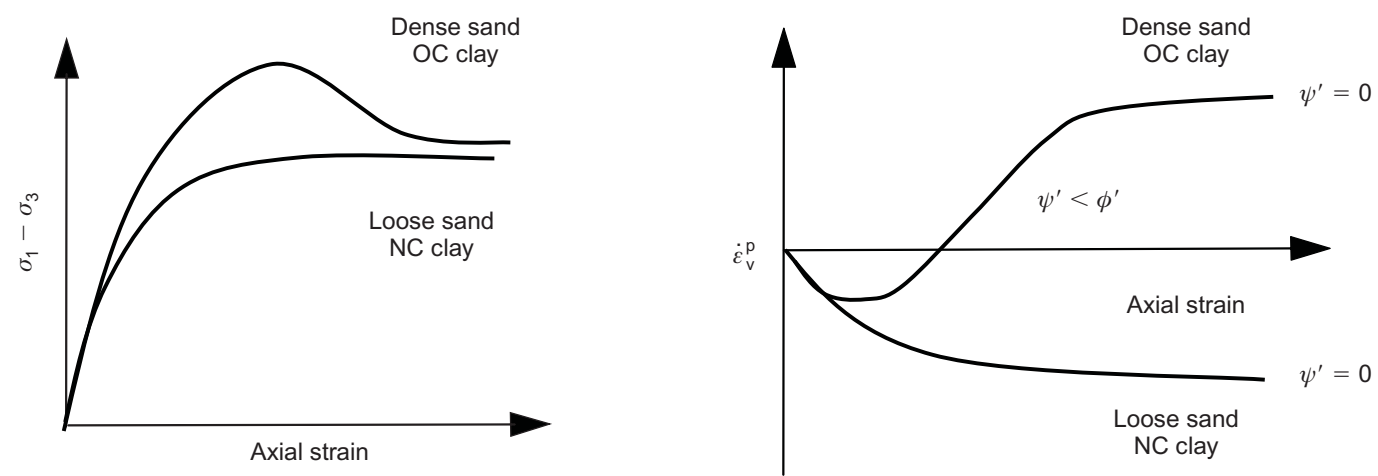

Fig. 37. Drained triaxial test behaviour 
$u_{x}=0, u_{y}$ prescribed
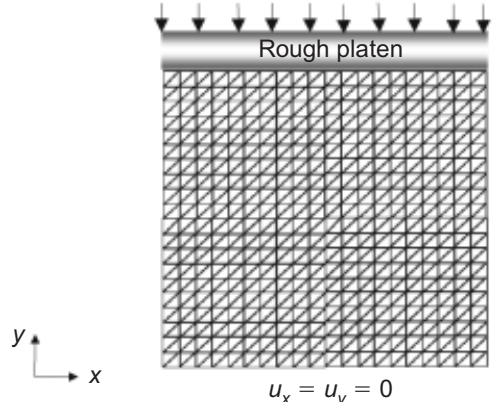

of the fact that the failure plane cannot form at an angle of $\theta=45^{\circ}+\psi^{\prime} / 2$ without intersecting the end platens, which causes the deformation field to be kinematically constrained. The numerical instability shown in Fig. 40 is not unusual for displacement finite-element analysis with a non-associated Mohr-Coulomb model, and can be especially severe when the friction angle is large and $\psi^{\prime} \ll \phi^{\prime}$ (e.g. De Borst \& Vermeer, 1984).

To investigate the collapse pressures and failure mechanisms for the cases with an associated flow rule, the biaxial test problem was reanalysed using discrete limit analysis with adaptive grid refinement and a maximum of 20000 elements. The results for the short specimen with $L / B=1$, shown in Fig. 41, bracket the exact collapse pressure to within $1 \%$, so that $4 \cdot 48 c^{\prime} \leqslant q_{\mathrm{u}} \leqslant 4 \cdot 53 c^{\prime}$. The corresponding failure mechanism, as indicated by the power dissipation contours, is clearly constrained to intersect the edges of the loading platens, and is highly localised. In contrast, for the longer specimen with $L / B=3$, the failure mechanism is more diffuse and shows no tendency to localise, even with a mesh of 20000 elements. The predicted collapse pressure for this case matches the exact analytical value of $q_{\mathrm{u}} / c^{\prime}=2 \tan \left(45^{\circ}+\phi^{\prime} / 2\right)$.

Finally, to investigate the potential of using the reduced strength parameters proposed by Davis (1968), equation (38) was employed to evaluate $c^{*}$ and $\phi^{*}$ for the short nonassociated sample with $c^{\prime}=1, \phi^{\prime}=30^{\circ}$ and $\psi^{\prime}=15^{\circ}$. The resulting strength parameters $c^{*}=0.96$ and $\phi^{*}=29.02^{\circ}$ were then adopted to estimate the collapse pressure using the adaptive discrete limit analysis methods with a cap of 20000 elements. This process gave a collapse pressure in the range $4 \cdot 12 c^{\prime} \leqslant q_{\mathrm{u}} \leqslant 4 \cdot 16 c^{\prime}$, which is approximately $8 \%$ below the best estimate of $q_{\mathrm{u}} \approx 4.47 c^{\prime}$ from the nonassociated displacement finite-element results shown in Fig. 40. Bearing in mind the uncertainty that is linked to the non-associated displacement finite-element results, this suggests that the 'Davis parameters' defined by equation (38) may provide a viable option for predicting the limit loads for frictional materials with non-associated flow rules. Indeed, this option will be explored further in the next section, and is supported independently by the recent work of Krabbenhøft et al. (2012).

and , for the non-associated models, exhibit oscillati after the onset of plastic deformation. In the most extreme case of a strongly non-associated flow rule with zero plastic volume change $\left(\psi^{\prime}=0^{\circ}\right)$, the numerical solution process becomes unstable at a total vertical strain of around $0.8 \%$. This difference in sensitivity to the flow rule is a direct result

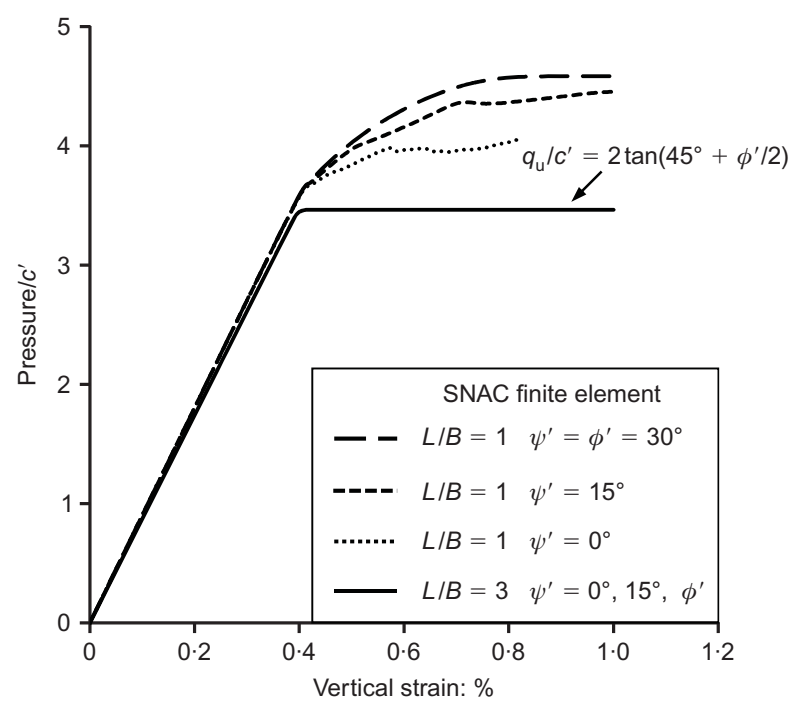

Fig. 40. Displacement finite-element results for biaxial compression of Mohr-Coulomb block

\section{APPLICATIONS: DRAINED STABILITY ANALYSIS}

The cases considered in the section on 'Undrained stability analysis' demonstrated the potential and versatility of finite-element limit analysis for predicting the stability of a wide range of problems under undrained conditions. In this section, these methods are applied to problems involving drained loading for both purely frictional and cohesivefrictional Mohr-Coulomb materials.

Soil anchors are widely used to provide uplift or lateral resistance for structures such as transmission towers, sheetpile walls and buried pipelines. Although most plate anchors are usually square, circular or rectangular in shape, many existing solutions have been developed for plane-strain strips, since these are significantly easier to analyse. A comprehensive survey of solutions that are available for predicting the capacity of various types of anchor in sand can be found in Merifield et al. (2006a) and Merifield \& Sloan (2006). These authors also summarise the results of a large number of chamber, centrifuge and field tests that can be used to verify theoretical predictions.

Here the pullout capacity, $Q_{\mathrm{u}}$, of a rough circular anchor in sand with unit weight $\gamma$ and friction angle $\phi^{\prime}$ is consid-

\section{Pullout capacity of rough circular anchor in sand}



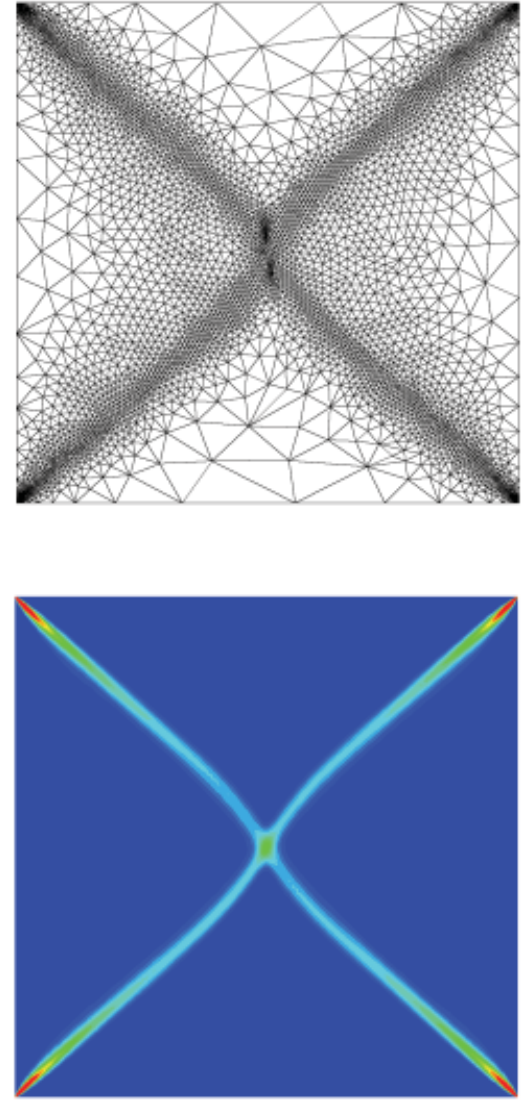

$$
\begin{aligned}
& L / B=1 \\
& c^{\prime}=1 \\
& \phi^{\prime}=\psi^{\prime}=30^{\circ} \\
& 4 \cdot 48 \leqslant q_{\mathrm{u}} / c^{\prime} \leqslant 4 \cdot 53
\end{aligned}
$$
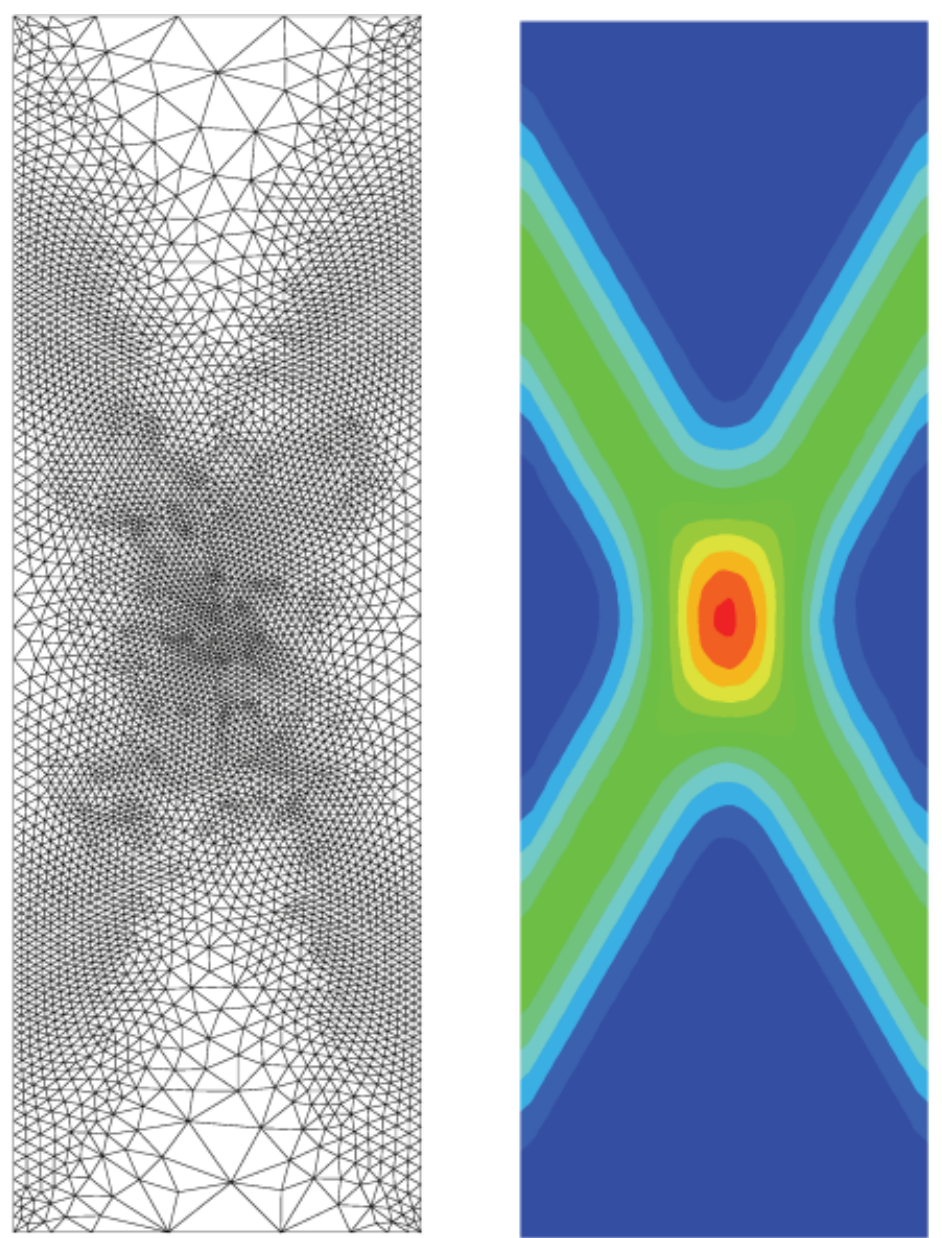

$$
\begin{aligned}
& L / B=3 \\
& c^{\prime}=1 \\
& \phi^{\prime}=\psi^{\prime}=30^{\circ} \\
& q_{\mathrm{u}} / c^{\prime}=3 \cdot 46=\text { exact }
\end{aligned}
$$

Fig. 41. Finite-element limit analysis meshes and failure mechanisms for biaxial compression of Mohr-Coulomb block (associated flow rule)

ered, as shown in Fig. 42. For an anchor of diameter $D$ buried at depth $H$, the ultimate load capacity can be expressed in the form $Q_{\mathrm{u}}=\gamma H A N_{\gamma}$, where $A=\pi D^{2} / 4$ is the anchor area, and $N_{\gamma}$ is a dimensionless 'breakout' factor that is a function of $\phi^{\prime}$ and $H / D$. Fig. 42 illustrates the finiteelement limit analysis mesh used for an anchor with $H$ / $D=2$. Even though the anchor problem is axisymmetric in nature, a three-dimensional slice is analysed to obtain fully rigorous upper- and lower-bound solutions that properly account for the hoop components of velocity and stress. The number of tetrahedra used in the limit analysis calculations ranged from approximately 2000 (for $H / D=2$ ) to 14000 (for $H / D=10$ ), with corresponding CPU times of 5-80 min. In all lower-bound analyses, three-dimensional extension elements were employed to extend the stress field over the semi-infinite domain (these are not shown). To estimate $N_{\gamma}$ for each geometry, the vertical pullout force $Q_{\mathrm{u}}$ was optimised directly after specifying the material properties and anchor dimensions.

For the case of an anchor in a medium-dense sand with $\phi^{\prime}=43 \cdot 1^{\circ}$ and $\psi^{\prime}=13 \cdot 6^{\circ}$, Fig. 43 shows the breakout factor $N_{\gamma}$ predicted from discrete limit analysis, as well as the SNAC displacement finite-element code. In the former set of analyses, the upper and lower bounds on $N_{\gamma}$ were computed by adopting the reduced friction angle of $\phi^{*}=38.4^{\circ}$, defined by Davis' equation (38), to account for the influence of non-associated flow. Fig. 43 also shows the laboratory test results reported by Pearce (2000) for a sand with an identical friction angle and dilation angle, and by Ilamparuthi et al. (2002) for a sand with a friction angle of $\phi^{\prime}=43^{\circ}$. Overall, discrete limit analysis provides good predictions of $N_{\gamma}$ for all anchor depths, although there is some discrepancy with the observations of Pearce (2000) for high values of $H / D$ (where the author reported that the effects of his chamber dimensions could be significant). Indeed, although they are preliminary, these limit analysis results provide encouraging support for the option of using Davis' reduced strengths for soils with high friction angles, where the influence of non-associated flow is most likely to be significant. Interestingly, the limit analysis estimates of $N_{\gamma}$ also compare well with the displacement finite-element predictions, which were based on the actual measured friction and dilation angles of $\phi^{\prime}=43 \cdot 1^{\circ}$ and $\psi^{\prime}=13 \cdot 6^{\circ}$. It should be noted, however, that considerable judgement was needed to determine the values for $N_{\gamma}$ from some of the displacement finite-element computations, owing to oscillations in the load-deformation response. These oscillations were similar in magnitude to those in observed in Fig. 40 for the non-associated analyses of the biaxial test, and in a few cases led to numerical problems associated with poor convergence. No such problems occur when the 'Davis parameters' are adopted in the limit analysis formulations, since these assume an associated flow rule.

For a cohesionless material such as sand, the quantity to 

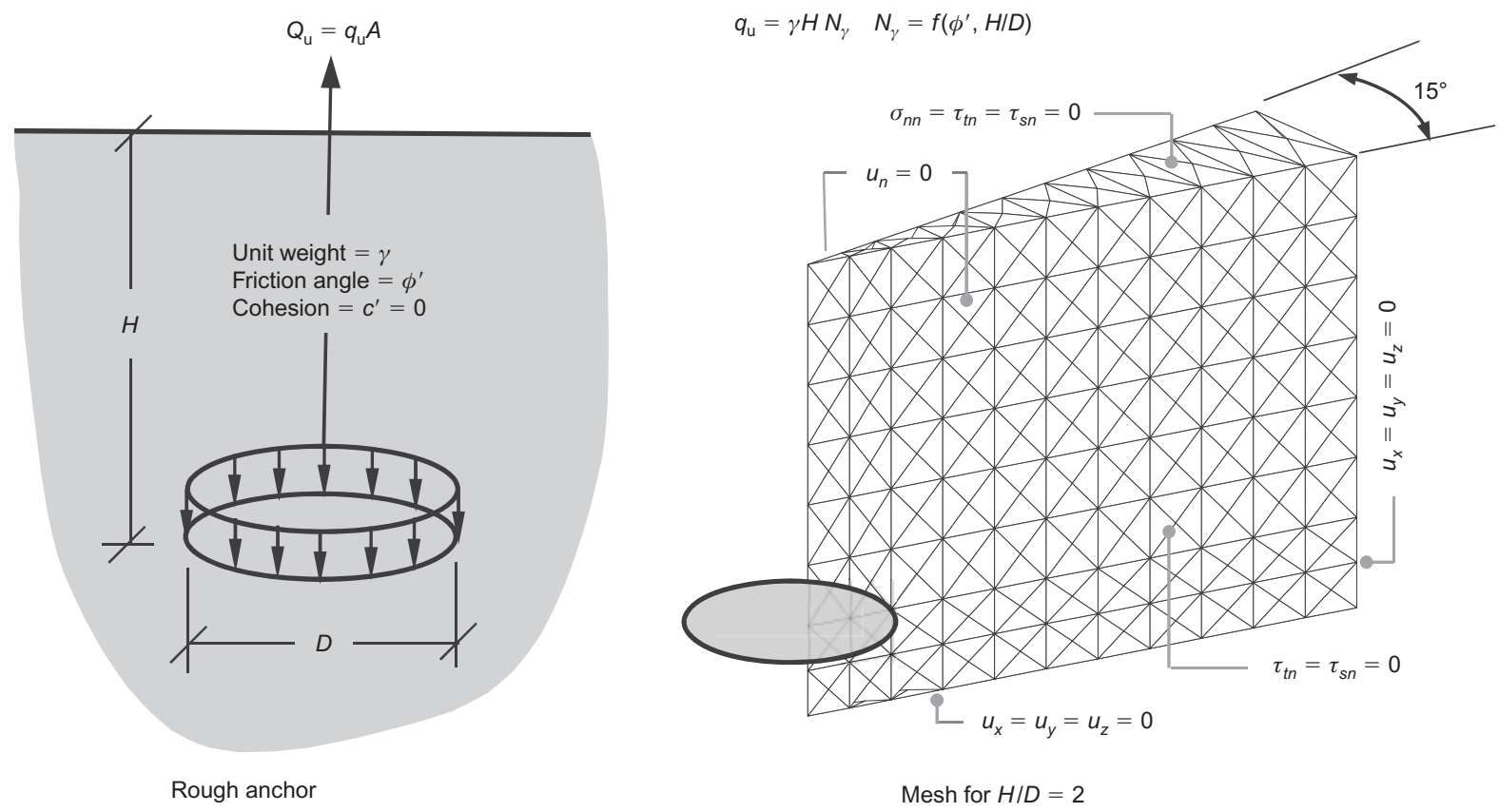

Fig. 42. Circular anchor in sand: problem definition and limit analysis mesh

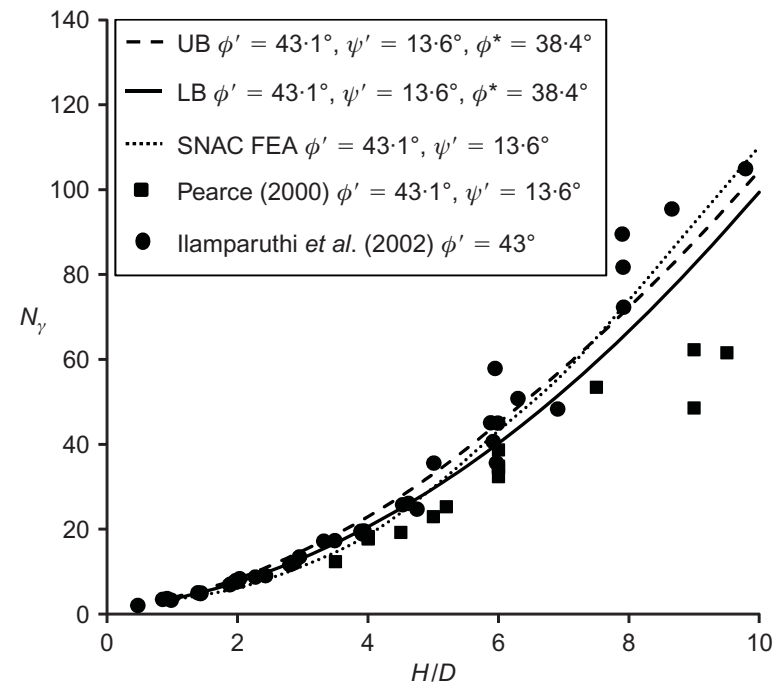

Fig. 43. Comparison of limit analysis and displacement finiteelement predictions with chamber test results for circular anchor in sand

be minimised in an upper-bound calculation is simply the rate of work done by any set of fixed external tractions or body forces, since the internal dissipation, defined by equation (16), is identically zero. To visualise the failure mechanism for this type of material it is convenient to plot contours of the plastic multipliers, since these indicate the magnitudes of the plastic strain rates and thus can be used to identify zones of intense plastic deformation. Two such plots for a circular anchor in medium-dense sand with $\phi^{\prime}=\psi^{\prime}=35^{\circ}$ are shown in Fig. 44. Both the shallow $(H / D=2)$ and deep $(H / D=10)$ cases show clearly defined failure mechanisms and yield bounds on $N_{\gamma}$ that differ from their averages by $\pm 6 \%$ and $\pm 4 \%$ respectively.
Stability of an unsupported circular excavation in cohesivefrictional material

The stability of an unsupported circular excavation, of depth $H$ and radius $R$, in a cohesive-frictional Mohr-Coulomb material is now considered (Fig. 45). Like the previous anchor example this case is axisymmetric, but was treated using a three-dimensional $15^{\circ}$ slice to account properly for the hoop terms in the bound calculations. To simplify the study the same meshes were used for the upper- and lower-bound analyses, except that extension elements were not required in the former. The stability number for the excavation, $\gamma H / c^{\prime}$, was found by optimising the unit weight $\gamma$ after fixing the cohesion $c^{\prime}$, the friction angle $\phi^{\prime}$ and the ratio $H / R$. This example thus illustrates the benefits of being able to optimise a body force directly in the discrete limit analysis formulations.

Figure 46 shows finite-element limit analysis solutions for the stability number $\gamma H / c^{\prime}$ where $H / R=1,2,3$ and $\phi^{\prime}=0^{\circ}$, $10^{\circ}, 20^{\circ}$. For the deepest excavation the upper and lower bounds differ from their average by a maximum of $\pm 2.5 \%$, while for the shallowest excavation the bounds are even closer, with a difference of less than $\pm 0.5 \%$.

Also shown in Fig. 46 are solutions for the purely cohesive case derived by Britto \& Kusakabe (1982), Pastor \& Turgeman (1982) and Turgeman \& Pastor (1982). Britto \& Kusakabe's upper bounds, obtained from an axisymmetric mechanism, compare reasonably well with the finite-element limit analysis results for all the geometries considered. Similarly, the upper-bound solution of Turgeman \& Pastor (1982), found from an axisymmetric finite-element formulation based on linear programming, also gives a good estimate of $\gamma H / c^{\prime}$ for the case $H / R=1$.

The influence of the friction angle on the shape of the failure mechanism can be seen from Fig. 47, which shows contour plots of the element plastic multipliers for the deepest excavation with $\phi^{\prime}=0^{\circ}$ and $\phi^{\prime}=20^{\circ}$. As expected, the zone of plastic deformation is much more extensive for the purely cohesive case, being roughly twice as wide as that for the excavation with $\phi^{\prime}=20^{\circ}$. 

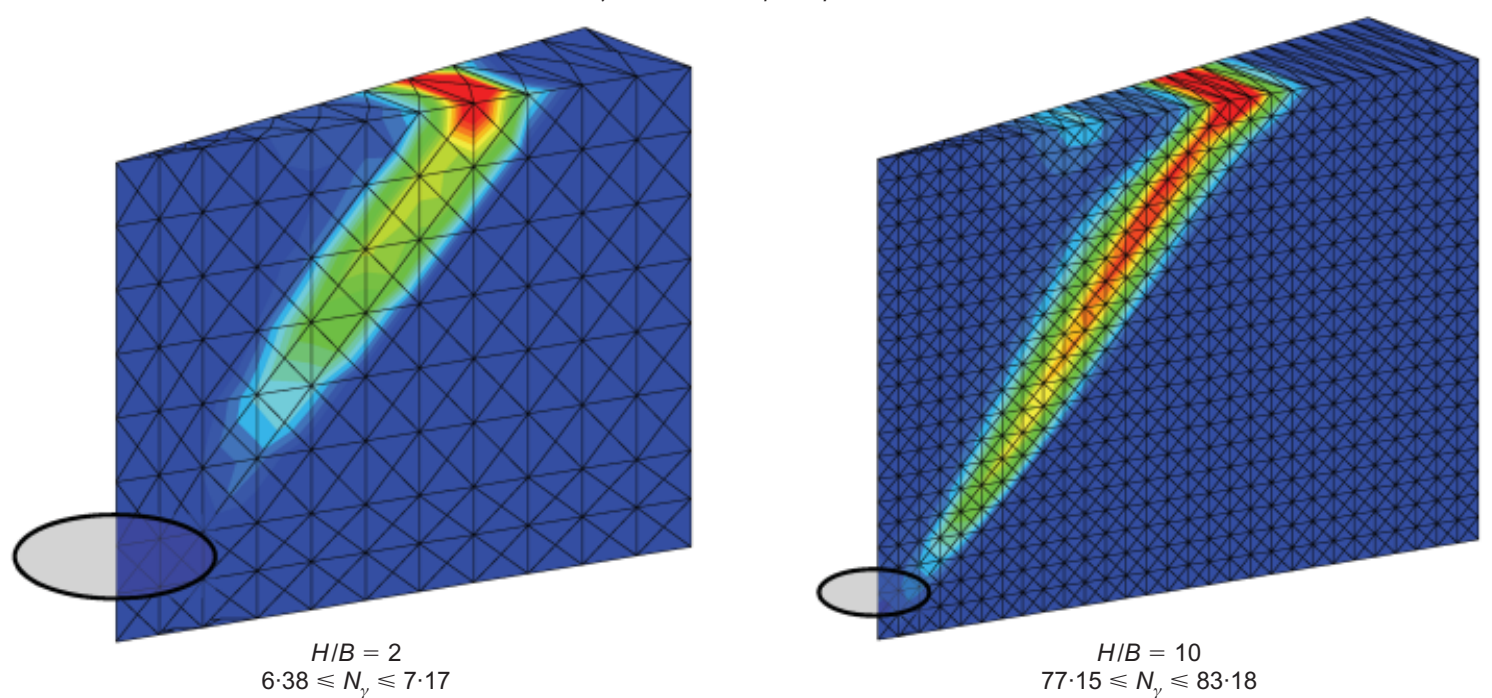

Fig. 44. Plastic multiplier (strain) contours for shallow and deep circular anchors in sand


Fig. 45. Circular excavation in cohesive-frictional soil: problem definition and limit analysis mesh

\section{INCORPORATION OF PORE PRESSURES IN LIMIT ANALYSIS}

Pore water pressures have a major effect on the stability of many geotechnical structures, and it is important that they are properly accounted for. In this section, a new approach is described that incorporates the effects of steady-state seepage in finite-element limit analysis. To find the steadystate pore pressures, the governing seepage equation is solved using optimisation theory and finite elements. Both confined and unconfined seepage flow conditions are modelled efficiently, and the problem of locating the phreatic surface in the latter presents no special difficulty. Since the proposed method employs the same mesh as the upper- and lower-bound analyses, there is no need to import and interpolate the pore pressures from another grid (or program), which is a significant practical benefit.

During the iterative solution process, a Hessian (curvature)-based error estimator is applied to the pore pressure field to generate a mesh that gives accurate pore pressures. Simultaneously, the 'bounds gap' error estimator of the section 'Adaptive mesh refinement' is employed to identify a separate mesh that gives accurate upper and lower bounds on the limit load. By combining these two strategies, a hybrid refinement strategy is developed that minimises both the bounds gap and the error in the computed pore pressures. 


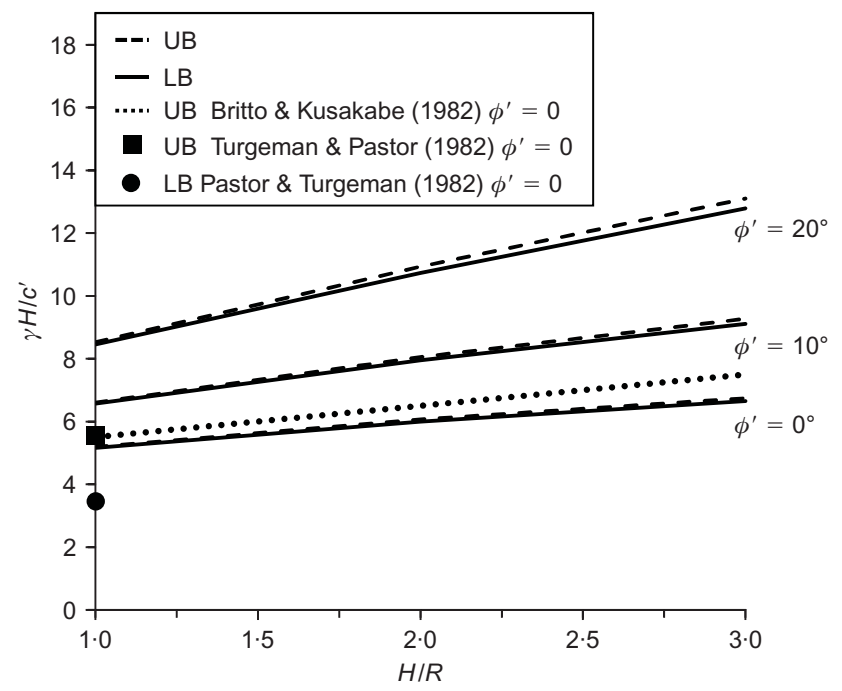

Fig. 46. Stability of unsupported circular excavation in cohesivefrictional soil

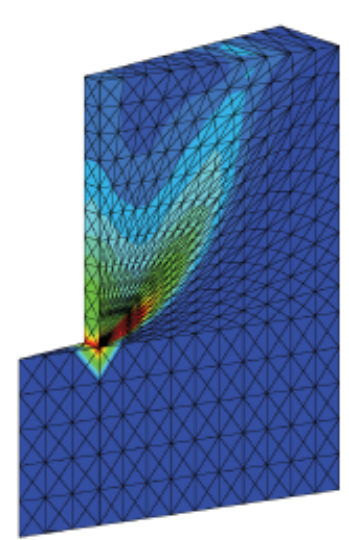

$\phi^{\prime}=0^{\circ}, H / R=3$

$6 \cdot 65 \leqslant \gamma H / c^{\prime} \leqslant 6 \cdot 74$

Fig. 47. Plastic multiplier (strain) contours for collapse of circular excavation

\section{Determination of steady-state pore pressures}

When seepage flow is present, the pressure head needs to be found in order to compute the pore pressure and effective stress at any point. Thus, during each iteration, the relevant seepage problem must first be solved before the stability analysis can be carried out. In the case of confined flow all the boundary conditions are known a priori, and the hydraulic head can be found by solving the governing seepage equation. For unconfined seepage, however, the conditions on some sections of the boundary are unknown, and must be determined as part of the solution. An example of the latter case is the flow of water through an earth dam, where the hydraulic head on either side of the dam is known but the precise location of the phreatic surface within the dam is not. Interestingly, both types of flow can be modelled in a single optimisation formulation that is based on a variational inequality (e.g. Crank, 1984).

By combining the fluid balance equations with Darcy's law for two-dimensional seepage through an isotropic porous medium, the governing equation for the total head $H$ is obtained as

$$
k(x, y) \nabla^{2} H=k(x, y) \frac{\partial^{2} H}{\partial x^{2}}+k(x, y) \frac{\partial^{2} H}{\partial y^{2}}=0
$$

where $k(x, y)$ is the soil permeability. Using standard vari- ational calculus, the solution to this equation can be written as the optimisation problem

$$
\text { Minimise } \frac{1}{2} \int_{V}(\nabla H)^{\mathrm{T}} k \nabla H \mathrm{~d} V
$$

subject to appropriate boundary conditions on the total head $H$.

For the two-dimensional case, this problem can be discretised using the linear triangular element shown in Fig. 48 according to

$$
H=\sum_{i=1}^{3} N_{i} H^{i}=\mathbf{N}^{e} \boldsymbol{H}^{e}
$$

where $N_{i}$ are linear shape functions, $\mathbf{N}^{e}=\left[N_{1}, N_{2}, N_{3}\right]$ is the element shape function matrix, and $\boldsymbol{H}^{e}=\left\{H^{1}, H^{2}, H^{3}\right\}^{\mathrm{T}}$ is the element vector of unknown nodal heads. Substituting the expression for $H$ from equation (40) into equation (39) gives, after some manipulation, the discrete optimisation problem

$$
\begin{array}{lc}
\text { Minimise } & \frac{1}{2} \boldsymbol{H}^{\mathrm{T}} \mathbf{K} \boldsymbol{H} \\
\text { subject to } & \mathbf{A} \boldsymbol{H}=\boldsymbol{H}_{0}
\end{array}
$$

where $\boldsymbol{H}$ is a global vector of unknown nodal heads, $\mathbf{A}$ is a matrix describing the constant head boundary conditions, and $\mathbf{K}$ is a flow matrix defined by

$$
\mathbf{K}=\sum_{e}^{E} \int_{A^{e}} \nabla \mathbf{N}^{e \mathrm{~T}} k(x, y) \nabla \mathbf{N}^{e} \mathrm{~d} A
$$

in which $E$ is the number of triangular elements, and $\nabla \mathbf{N}^{e}$ denotes the gradient of the shape function matrix for element $e$. Using numerical integration to evaluate $\mathbf{K}$, the solution to the quadratic optimisation problem in equation (41) is straightforward, and is defined by the linear relations

$$
\mathbf{K H}=\mathbf{0}
$$

subject to the boundary conditions $\mathbf{A} \boldsymbol{H}=\boldsymbol{H}_{0}$. Note that, in the formulation used here, which employs the same mesh for the pore pressure and the limit analysis calculations, the matrix $\mathbf{A}$ also contains terms to enforce continuity of the head across the discontinuities between adjacent elements. After solving equation (43) for the total head at each node, the corresponding pore pressures, $\boldsymbol{p}$, are readily obtained as $\boldsymbol{p}=(\boldsymbol{H}-\boldsymbol{z}) \gamma_{\mathrm{w}}$, where $\boldsymbol{z}$ is a vector of the nodal elevation heads and $\gamma_{\mathrm{w}}$ is the unit weight of water.

For problems involving unconfined seepage flow, the quadratic optimisation problem (equation (41)) is augmented by the constraint $\boldsymbol{p}=(\boldsymbol{H}-\boldsymbol{z}) \gamma_{\mathrm{w}} \geqslant \boldsymbol{O}$. This additional condition can be used to compute the pore pressures and phreatic surface using the following algorithm.

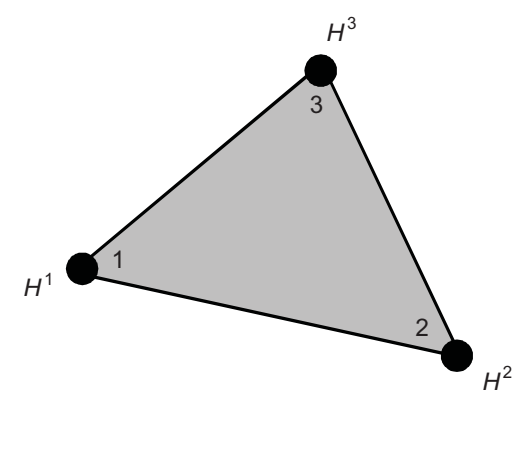

Fig. 48. Linear finite-element for modelling total head 
1. Solve equation (43) to give the nodal heads $\boldsymbol{H}$.

2. Compute the nodal pore pressures using the relation $\boldsymbol{p}=(\boldsymbol{H}-\boldsymbol{z}) \gamma_{\mathrm{w}}$.

3. If the change in objective function $\boldsymbol{H}^{\mathrm{T}} \mathbf{K} \boldsymbol{H}$ is less than a small tolerance, exit with the final pore pressures.

4. For all nodes $i$ where the pore pressure $p_{i}<0$, adjust the nodal permeability using the relation $\bar{k}_{i}=s(p) k_{i}$, where $s(p)$ is a smoothed step function that ranges between 0 and 1 (see Fig. 49).

5. Recompute $\mathbf{K}$ using equation (42) with the adjusted nodal permeabilities for each element $\bar{k}_{i}$; then go to step 1 .

This process, although relatively crude, typically locates the phreatic surface in five or six iterations, and thus imposes only a small overhead on the overall limit analysis computation. The smoothed step function in step 4 is introduced to minimise the occurrence of pore pressure oscillations in the vicinity of the phreatic surface. This function can take a variety of forms, although the simple expression $s(p)=\frac{1}{2}[1+\tanh (\alpha p)]$, shown in Fig. 49, works well in practice with $\alpha=50$. When computing the contributions to $\mathbf{K}$ in step 5, the permeability is assumed to vary linearly over each element. This gives a 'weighted' permeability for elements that are bisected by the phreatic surface, and aids convergence of the iteration scheme.

Lower-bound formulation with steady-state pore pressure

The inclusion of pore water pressure in the lower-bound method involves the use of effective stresses when enforcing the yield constraints, whereas total stresses are employed when imposing the equilibrium and stress boundary conditions. Since it uses the same mesh, the pore pressure is treated as an auxiliary variable that, like the effective stress, varies linearly over each element. This is shown for the two-dimensional case in Fig. 50. Note that, during the limit analysis calculations for each mesh, the pore pressure field is fixed.

Upper-bound formulation with steady-state pore pressure

The inclusion of pore pressure in the upper-bound method requires the use of effective stresses when enforcing the yield condition and flow rule. There is also an additional term in the governing equation (15) that is due to the rate of work done by the pore pressure field. The pore pressure field is again treated as an auxiliary variable that varies linearly over each element, as shown for the two-dimensional case in Fig. 51.

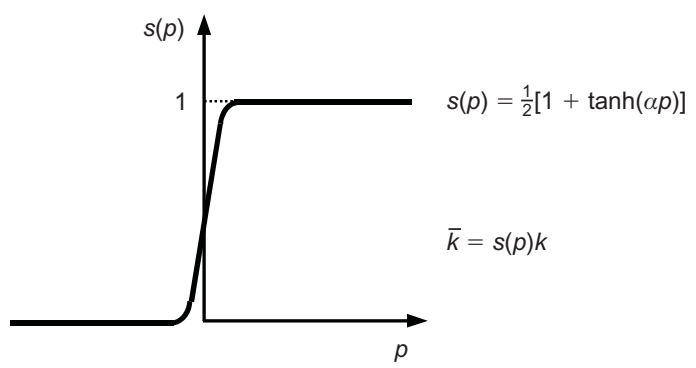

Fig. 49. Smoothed step function in permeability for locating phreatic surface

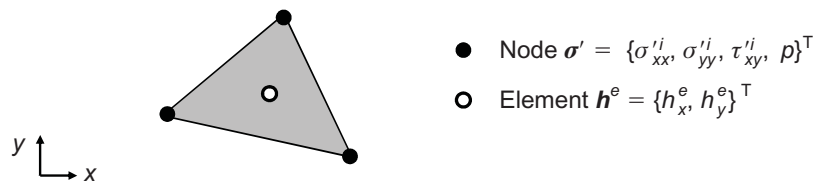

Fig. 50. Lower-bound element with auxiliary pore pressure

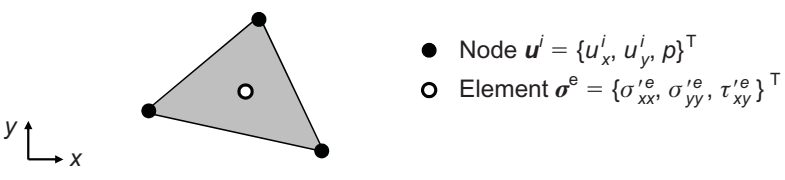

Fig. 51. Upper-bound element with auxiliary pore pressure

Following Kim et al. (1999), the additional term in equation (15) due to the rate of work done by the static pore pressures means that the quantity to be minimised becomes

$$
\dot{W}=\int_{V} \boldsymbol{\sigma}^{\mathrm{T}} \dot{\boldsymbol{\varepsilon}}^{\mathrm{p}} \mathrm{d} V-\int_{A_{t}} \boldsymbol{t}^{\mathrm{T}} \boldsymbol{u} \mathrm{d} A-\int_{V} \boldsymbol{g}^{\mathrm{T}} \boldsymbol{u} \mathrm{d} V-\int_{V} \nabla p^{\mathrm{T}} \boldsymbol{u} \mathrm{d} V
$$

where $\nabla p=\{\partial p / \partial x, \partial p / \partial y\}^{\mathrm{T}}$ is the gradient of the pore pressure field. Assuming that the pore pressure varies linearly, these derivatives are uniform over each element, and are given by the equations

$$
\begin{gathered}
\frac{\partial p}{\partial x}=\sum_{i=1}^{3} \frac{\partial N_{i}}{\partial x} p_{i}=\sum_{i=1}^{3} b_{i} p_{i} \\
\frac{\partial p}{\partial y}=\sum_{i=1}^{3} \frac{\partial N_{i}}{\partial y} p_{i}=\sum_{i=1}^{3} c_{i} p_{i}
\end{gathered}
$$

where $p_{i}$ are nodal pore pressures, and the constants $b_{i}$ and $c_{i}$ depend on the element nodal coordinates.

\section{Limit analysis with adaptive mesh refinement in presence of pore pressures}

As mentioned previously, a hybrid mesh refinement strategy can be developed that minimises the error in both the pore pressures and the upper and lower bounds. This is based on predicting good element sizes for the pore pressures using a Hessian (curvature)-based error estimator (Almeida et al., 2000), together with element sizes that directly minimise the bounds gap (as described in the section 'Adaptive mesh refinement'). Where the element sizes predicted by these two separate approaches differ, the hybrid scheme simply chooses the smallest one. Details of the Hessian-based scheme for selecting element sizes, in the context of lower-bound limit analysis, can be found in Lyamin et al. (2005b). Exactly the same approach is used here, with the 'isotropic' form of the method being implemented, which omits element 'stretching'.

The steps involved in performing finite-element limit analysis with adaptive mesh refinement, allowing for the presence of steady-state pore pressures, may be summarised as follows.

1. Specify the maximum number of continuum elements allowed, $E_{\max }$, and generate an initial mesh.

2. Compute the nodal pore pressures for the mesh using the algorithm described in the section 'Determination of steady-state pore pressures', allowing for unconfined flow if needed.

3. Perform upper-bound and lower-bound analyses using the same mesh as in step 2.

4. If the gap between the upper and lower bounds is less than a specified tolerance, or if the maximum number of continuum elements $E_{\max }$ is reached, exit with upper- and lower-bound estimates of the limit load.

5. Specify a target number of continuum elements for the current mesh iteration, $E_{i}$, with $E_{i} \leqslant E_{\max }$.

6. Using the nodal pore pressure field and the Hessian-based error estimator of Almeida et al. (2000), compute the 
optimum size of each element, subject to the constraint that the new number of continuum elements in the grid matches the predefined target number of continuum elements for the current iteration $E_{i}$.

7. For each element, compute its contribution to the bounds gap $\Delta^{e}$ using equation (37). In the case of a discontinuity element, its bounds gap contribution is added to the neighbouring continuum element with which it shares the most nodes. Then scale the size of each continuum element to be inversely proportional to the magnitude of $\Delta^{e}$, subject to the constraint that the new number of continuum elements in the grid matches the predefined target number of continuum elements for the current iteration $E_{i}$

8. Compare the predicted size for each element from steps 6 and 7, and choose the smallest one. Then scale the element sizes to meet the target number of continuum elements for the current iteration $E_{i}$.

9. Go to step 2 .

\section{APPLICATIONS: SLOPE STABILITY ANALYSIS}

Now the classical problem of slope stability is considered, and the solutions from finite-element limit analysis are compared with those found by conventional methods. Two cases are considered, one with no seepage flow and one with unconfined seepage flow, and both have a weak layer that causes a non-circular failure surface to develop. To permit direct comparisons with conventional methods of stability analysis, an efficient strength reduction scheme is described that gives the safety factor in terms of the shear strength rather than the applied load.

\section{Slope in cohesive-frictional soil with weak layer}

The first example, taken from the benchmark prediction exercise documented in Donald \& Giam (1989a), is shown in Fig. 52. The problem is designed to develop a noncircular failure plane that propagates along the weak zone, and is a useful test for conventional slope stability methods as well as finite-element limit analysis.

Using the algorithm described in the section 'Adaptive mesh refinement', adaptive finite-element limit analysis was performed with a maximum of 4000 continuum elements. Unlike previous examples, however, a strength reduction process was followed to compute the safety factor in terms of the shear strength (rather than the applied load). This process, shown graphically in Fig. 53, can be summarised by the following steps.

1. Start by assuming a trial safety factor, $F_{0}=1$.

2. Compute the available strengths $c_{\mathrm{a}}^{\prime}=c^{\prime} / F_{0}$ and $\phi_{\mathrm{a}}^{\prime}=\tan ^{-1}\left(\tan \phi^{\prime} / F_{0}\right)$.

3. Using the available strengths $\left(c_{\mathrm{a}}^{\prime}, \phi_{\mathrm{a}}^{\prime}\right)$ and the adaptive finite-element limit analysis algorithm given in the

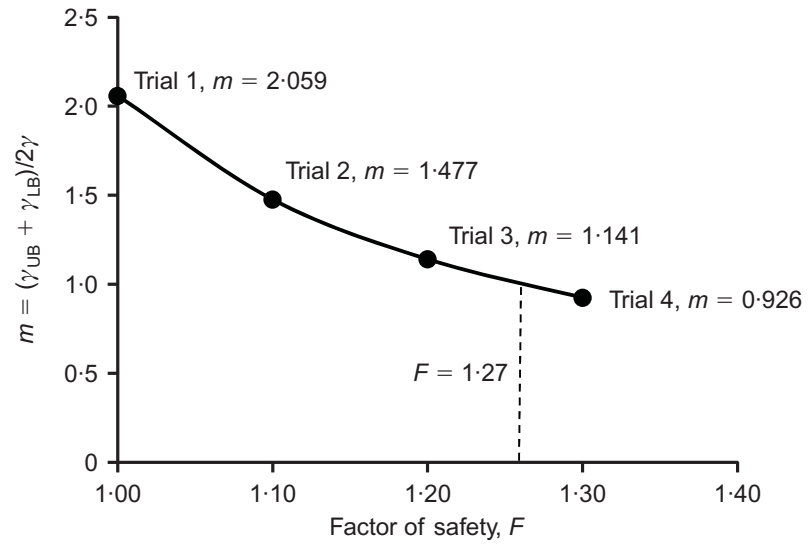

Fig. 53. Strength reduction process for slope with weak layer

section 'Adaptive mesh refinement', compute upper and lower bounds on the unit weight that can be supported by the slope $\left(\gamma_{\mathrm{LB}}, \gamma_{\mathrm{UB}}\right)$. Then compute the mean of these bounds according to $\bar{\gamma}=\left(\gamma_{\mathrm{UB}}+\gamma_{\mathrm{LB}}\right) / 2$ and the gravity multiplier $m_{0}=\bar{\gamma} / \gamma$, where $\gamma$ is the actual unit weight.

4. If $m_{0}<1$, set $\Delta F=-0 \cdot 1$; else set $\Delta F=0 \cdot 1$.

5. Compute $F_{1}=F_{0}+\Delta F$.

6. Compute the available strengths $c_{\mathrm{a}}^{\prime}=c^{\prime} / F_{1}$ and $\phi_{\mathrm{a}}^{\prime}=\tan ^{-1}\left(\tan \phi^{\prime} / F_{1}\right)$.

7. Using the available strengths $\left(c_{\mathrm{a}}^{\prime}, \phi_{\mathrm{a}}^{\prime}\right)$, compute upper and lower bounds on the unit weight $\left(\gamma_{\mathrm{LB}}, \gamma_{\mathrm{UB}}\right)$. Then compute the mean according to $\bar{\gamma}=\left(\gamma_{\mathrm{UB}}+\gamma_{\mathrm{LB}}\right) / 2$ and the multiplier $m_{1}=\bar{\gamma} / \gamma$.

8. If $\left(m_{1}-1\right)\left(m_{0}-1\right)>0$, then set $m_{0}=m_{1}$ and $F_{0}=F_{1}$ and go to step 5 .

9. Linearly interpolate the factor of safety according to $F=F_{0}+\left(F_{1}-F_{0}\right)\left(m_{0}-1\right) /\left(m_{0}-m_{1}\right)$.

This process starts by assuming a trial estimate of the safety factor, and continues with a simple marching scheme until the factor of safety is found that gives a gravity multiplier on the unit weight, $m$, of unity. Instead of taking the average of the upper and lower bounds on the unit weight to compute this multiplier in steps 3 and 7 , it is of course possible to use the actual lower or upper bounds, and hence compute an upper or lower bound on the safety factor $F$. This is an attractive feature, but it is generally unnecessary owing to the very tight bounds (better than $1 \%$ ) that are generated by the finite-element limit analysis approach. For this particular example, the safety factor $F=1.27$ was found after four iterations, and required around $30 \mathrm{~s}$ of CPU time.

The optimised mesh at the completion of the strength reduction process, shown in Fig. 54, indicates that the bounds gap error estimator has concentrated the elements along the failure surface, precisely where they are needed. The corresponding plots of the velocity vectors and plastic multipliers (strains), shown in Figs 55 and 56 respectively,

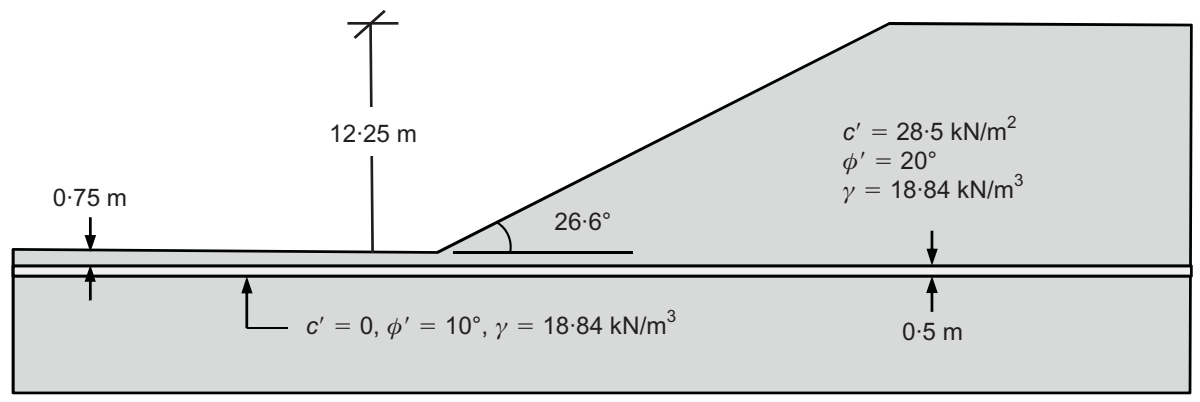

Fig. 52. Slope with weak layer: no seepage flow 


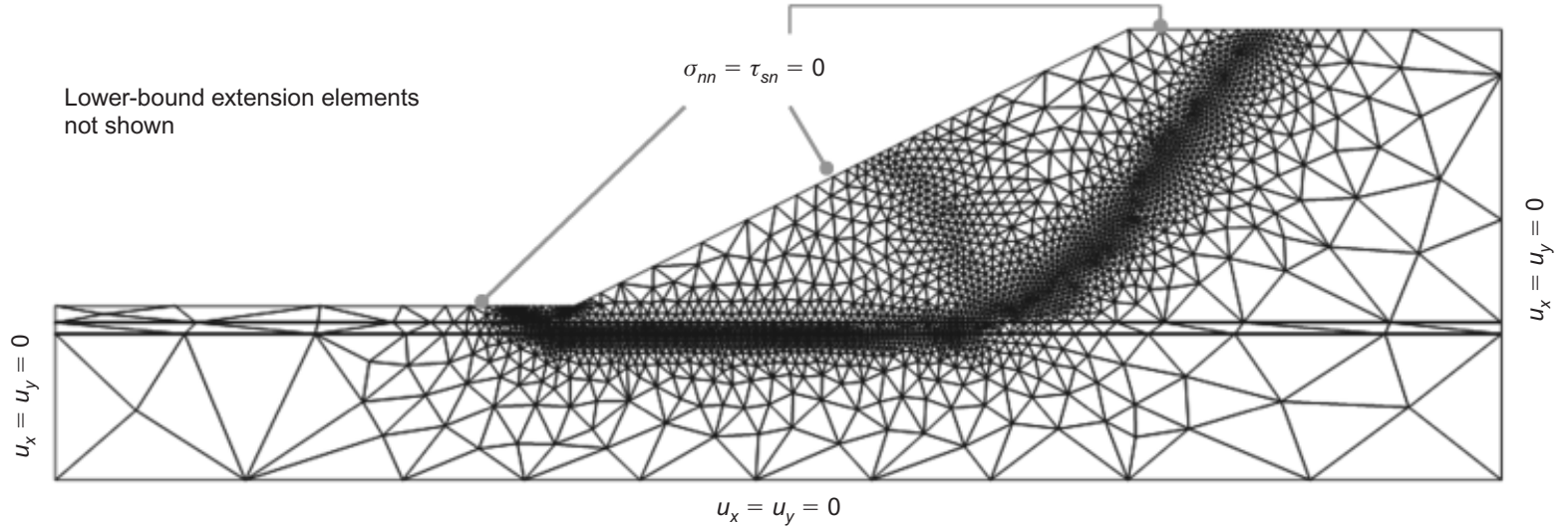

Fig. 54. Optimised mesh for slope with weak layer

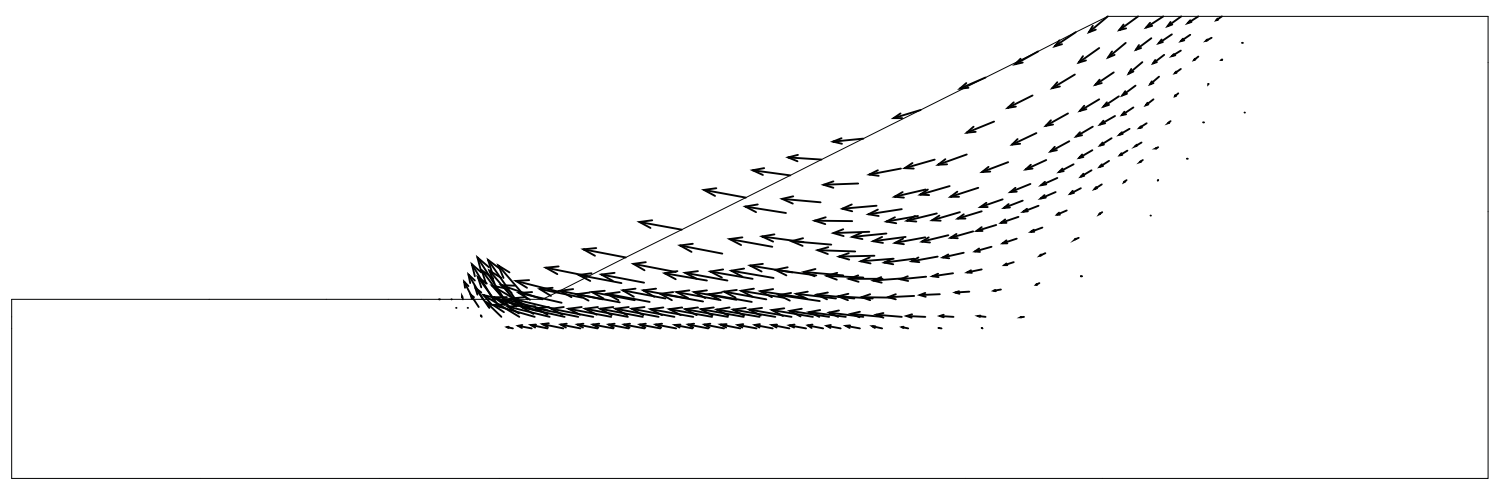

Fig. 55. Velocity vectors at collapse for slope with weak layer

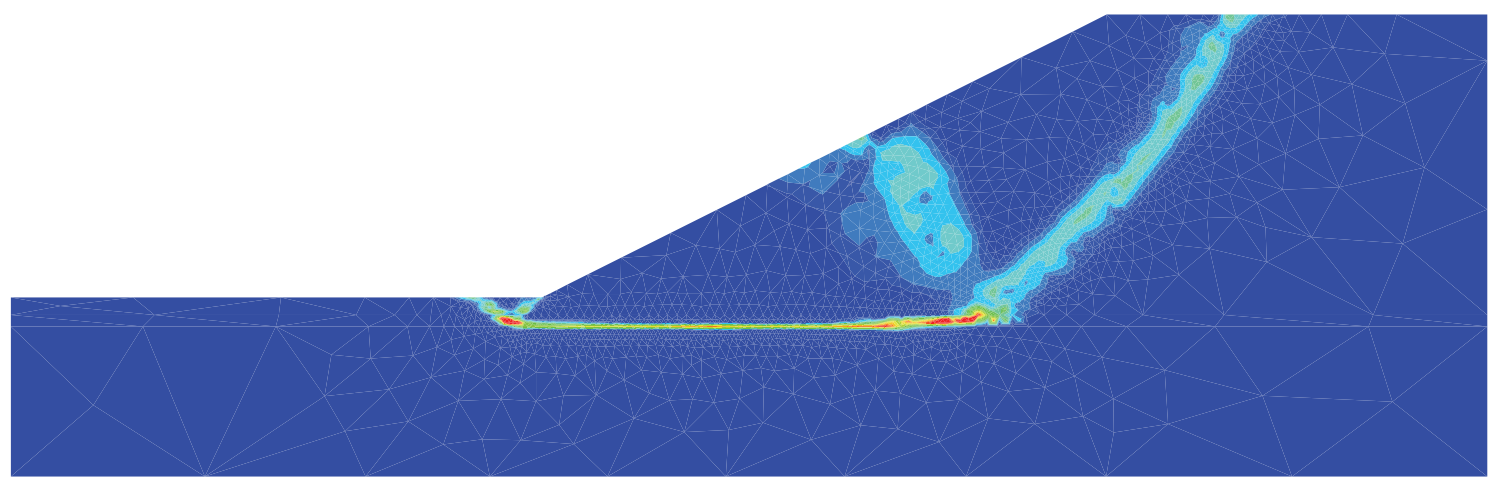

Fig. 56. Plastic multiplier (strain) contours at collapse for slope with weak layer

confirm that the mode of failure is dominated by intense shear deformation in the weak layer of cohesionless material. Interestingly, the latter plot indicates that a secondary failure mechanism also occurs along a plane at right angles to the slope face.

Figure 57 compares the factors of safety computed from finite-element limit analysis and a variety of conventional limit-equilibrium methods. The latter, reported in Donald \& Giam (1989a), indicate significant variations in the safety factor, even for analyses with the same procedure. These variations reflect the difficulty in locating the critical limitequilibrium failure surface, which is actually an unconstrained optimisation problem that demands sophisticated strategies to obtain a reliable solution (especially if the failure surface is permitted to be non-circular). In contrast, the solutions from the finite-element limit analysis method are guaranteed to give the best possible upper and lower bounds for a specified mesh, since the governing optimisation problem is both constrained and convex (provided the yield surface is convex).

A further complication with limit-equilibrium procedures is that they each make different assumptions in order to obtain a solution, some of which are physically more justified than others. This has resulted in a multitude of techniques being proposed in the literature, as well as endless debates on which one is the best. A detailed discussion of the theory and merits of various limit-equilibrium approaches can be found in Duncan \& Wright (2005). With regard to the results compared in Fig. 57, the methods of Morgenstern \& Price (1965), Spencer (1967) and Sarma (1973, 1979) may be viewed as 'complete equilibrium' techniques, since they satisfy both force and moment equilibrium for each slice. Compared with the limit analysis prediction of $F=1 \cdot 27$, the various implementations of the 


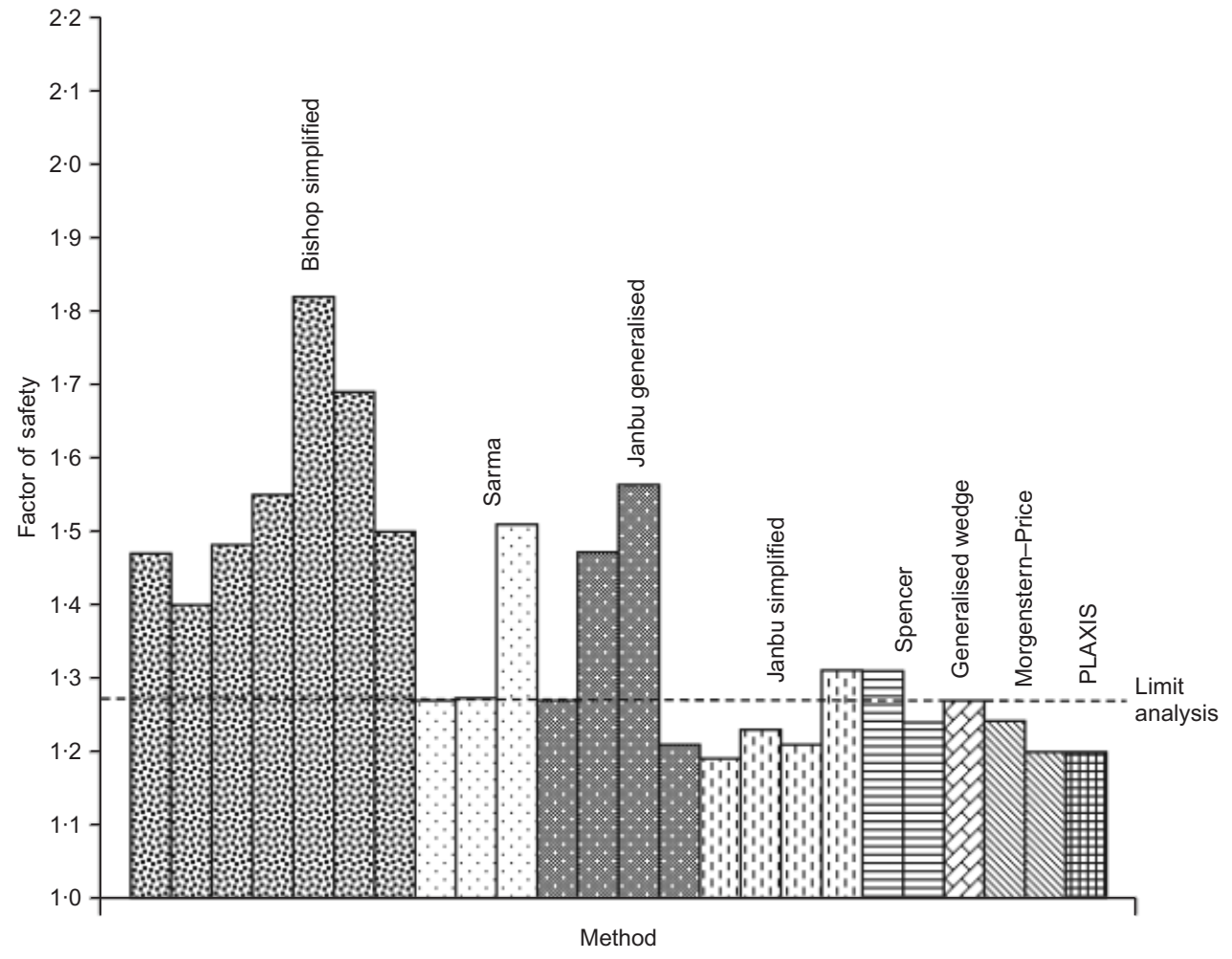

Fig. 57. Comparison of factors of safety for slope with a weak layer

Morgenstern-Price, Spencer and Sarma methods reported in Donald \& Giam (1989a) gave, respectively, $F=(1 \cdot 242,1 \cdot 2)$, $F=(1.31,1.24)$ and $F=(1.27,1.273,1.51)$. Hence, to three significant figures, two of the Sarma predictions coincide with those of the limit analysis method, whereas the average of the Spencer estimates is $F=1 \cdot 275$. The generalised wedge method of Donald \& Giam (1989b) also gives a safety factor of 1.27 . Interestingly, it can be shown (Giam \& Donald, 1989a) that this technique gives answers identical to those of the rigorous upper-bound wedge method of Giam \& Donald (1989b) and Donald \& Chen (1997), which further corroborates the finite-element limit analysis estimate. The overestimates of the safety factor provided by the simplified Bishop method reflect the fact that it is better suited to cases where the failure surface can be approximated by a circle.

For completeness, Fig. 57 also shows the factor of safety computed by the displacement finite-element code PLAXIS 2D (2011) using strength reduction. The estimate from this method of $F=1.20$ is slightly low, possibly because the program assumes non-associated flow for the Mohr-Coulomb model in the strength reduction iteration process. In using strength reduction with the displacement finite-element method, there is also the question of which monitoring points should be chosen to detect non-convergence of the iterations, as different points can give slightly different safety factors.

\section{Slope in cohesive-frictional soil with weak layer and} unconfined seepage flow

The final example is identical to the preceding case, except that the slope is now subject to the effects of pore pressures that are generated by unconfined seepage flow (Fig. 58). Ignoring, for the moment, the limit analysis phase, Figs 59 and 60 show, respectively, the optimised mesh and the pore pressure head generated by the methods described in 'Determination of steady-state pore pressures' and 'Limit analysis with adaptive mesh refinement in presence of pore pressures'. In these results, for a mesh with a maximum of 2000 elements, the Hessian-based refinement scheme clearly identifies the phreatic surface and concentrates the elements in its vicinity. Moreover, the contours of the pore pressure

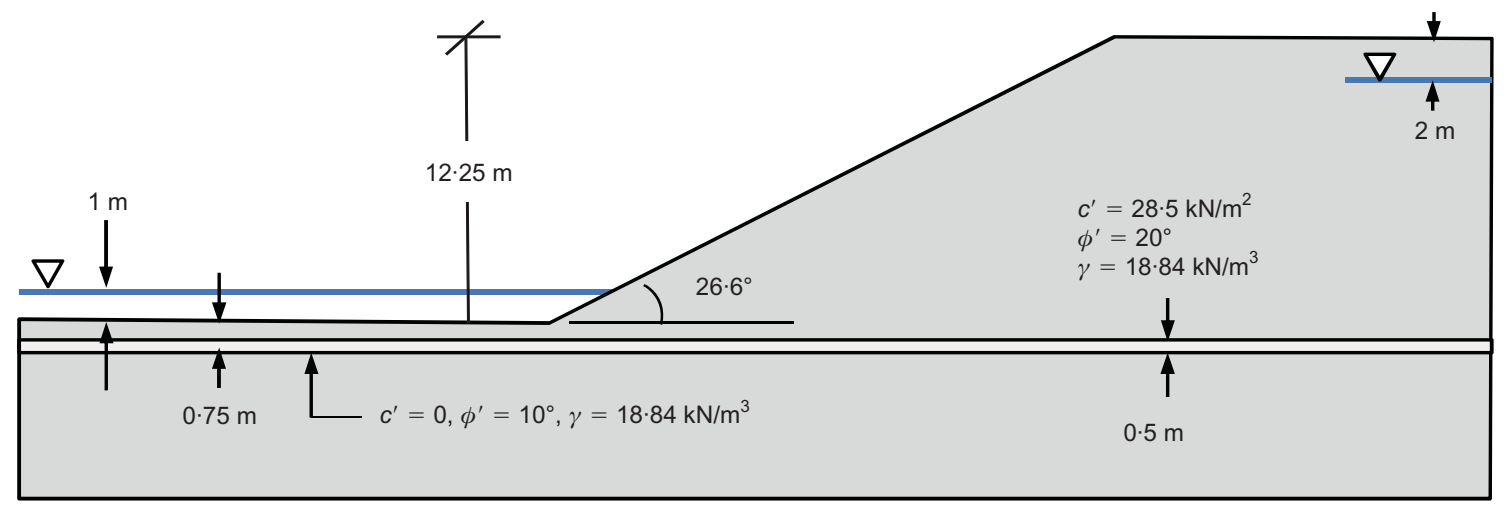

Fig. 58. Slope with weak layer: unconfined seepage flow 


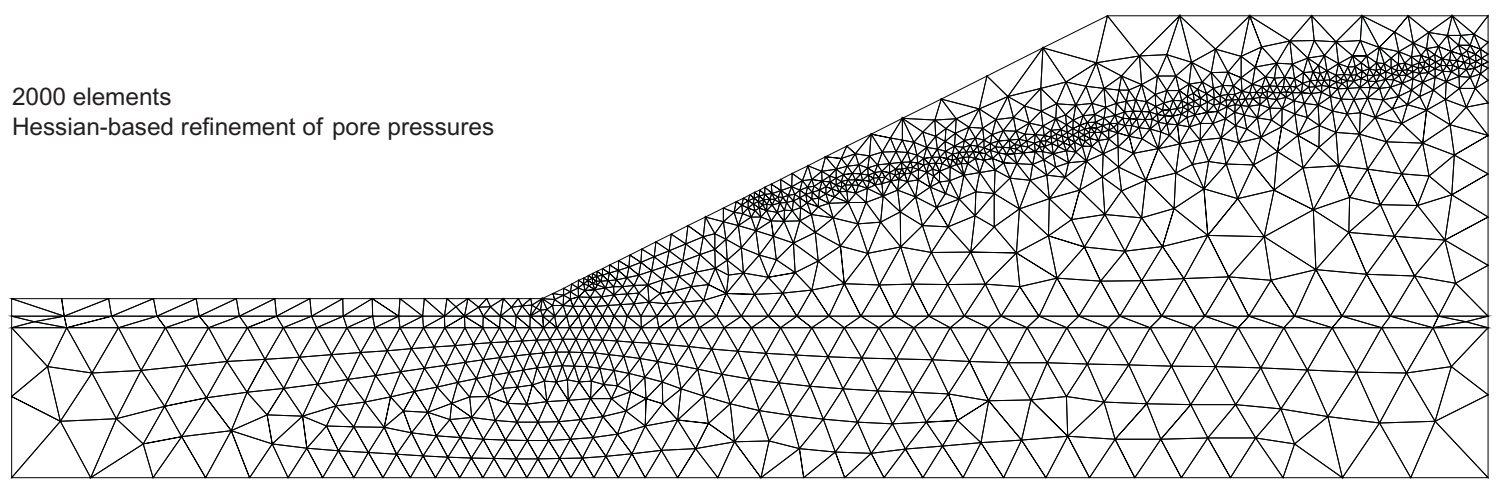

Fig. 59. Optimised pore pressure mesh for unconfined seepage flow in slope with weak layer

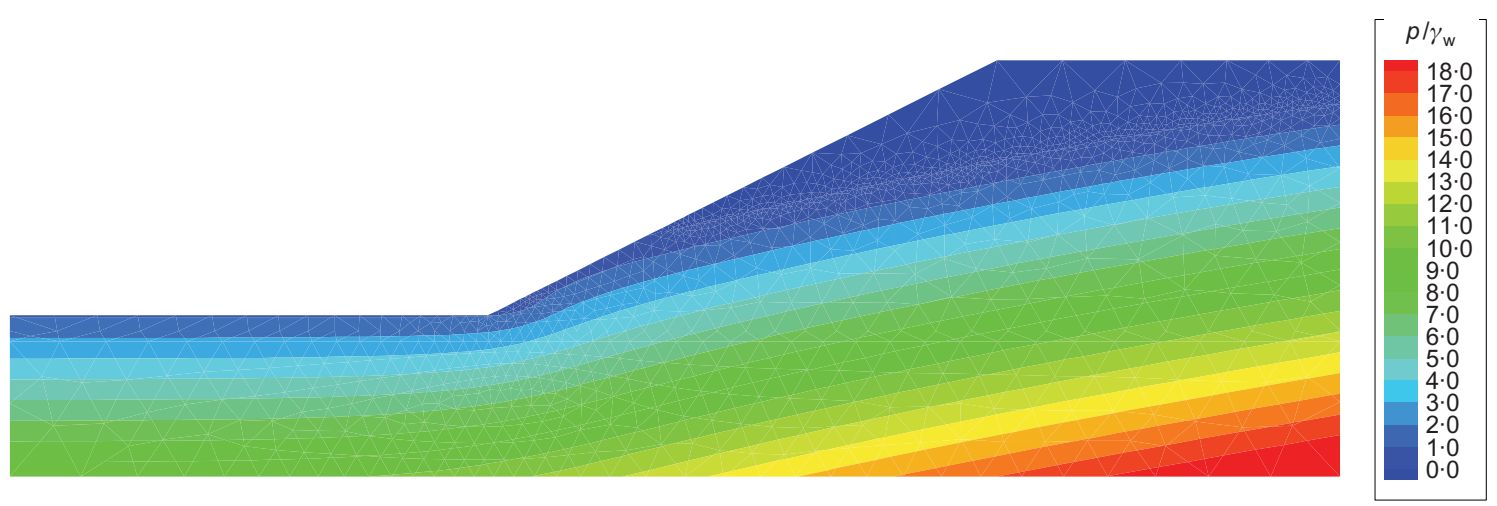

Fig. 60. Pore pressure head for unconfined seepage flow in slope with weak layer

head are smooth, and have values that were verified independently using the program SEEP/W in GeoStudio (2007).

The strength reduction process for this example is again conducted using the algorithm described in 'Slope in cohesive-frictional soil with weak layer and unconfined seepage flow', except that the hybrid mesh refinement scheme of 'Limit analysis with adaptive mesh refinement in presence of pore pressures', which accounts for pore pressures, is used in steps 3 and 7. Starting with an initial safety factor of unity, only two strength reduction trials are needed to identify the safety factor $F=0 \cdot 96$, as shown in Fig. 61 . This result was obtained with a maximum of 4000 elements in the adaptive limit analysis calculations, and required a total of around $14 \mathrm{~s}$ of CPU time. The optimised mesh at the completion of the strength reduction process, shown in Fig. 62 , indicates that the hybrid adaptivity scheme of 'Limit analysis with adaptive mesh refinement in presence of pore

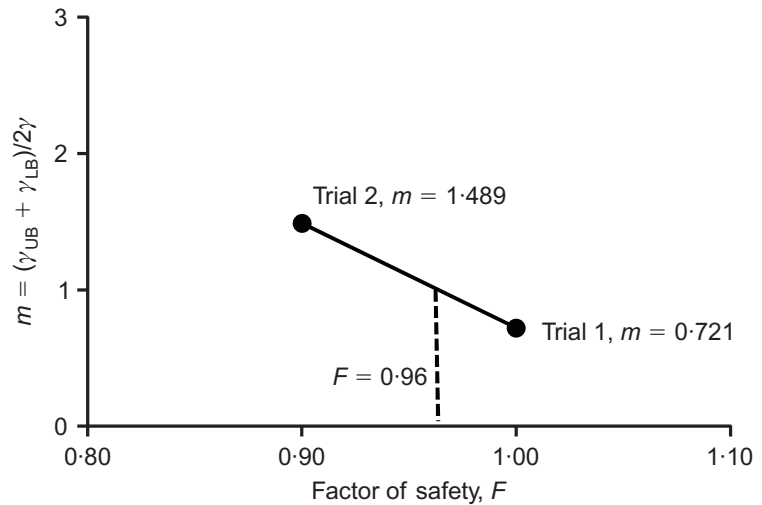

Fig. 61. Strength reduction process for slope with weak layer and unconfined seepage flow

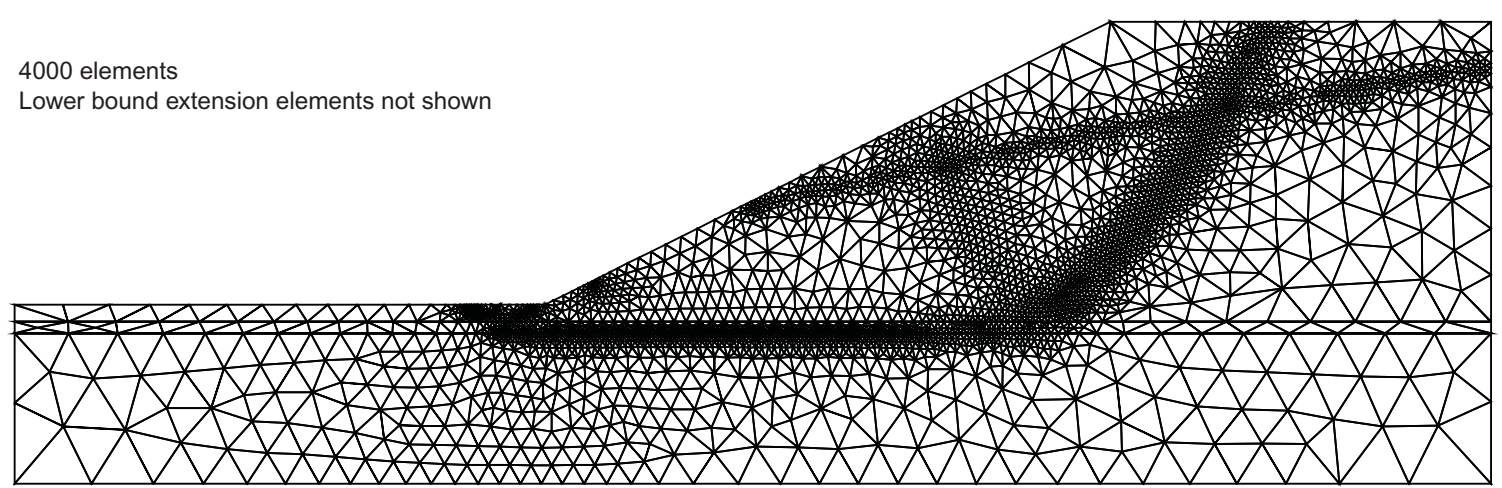

Fig. 62. Optimised mesh for slope with weak layer and unconfined seepage flow 
pressures' has, simultaneously, concentrated elements along the failure surface and the phreatic surface. The corresponding velocity vectors at collapse, shown in Fig. 63, confirm that the mode of failure is again dominated by intense shear deformation in the weak layer of cohesionless material. Compared with the case with no water table (Fig. 55), the failure mechanism is much more extensive, and generates greater lateral deformation on the face of the slope.

Figure 64 compares the factors of safety computed from finite-element limit analysis and a variety of conventional limit-equilibrium methods as implemented in SLOPE/W in GeoStudio (2007). To obtain the values for the latter, each method was run with a variety of options (where applicable) until the lowest factor was found. Compared with the limit analysis estimate of $F=0.96$, the SLOPE/W implementations of the Morgenstern-Price, Spencer and Sarma methods give values of $F=0.94, F=0.94$ and $F=0.95$ respectively. Slightly lower values are obtained from the less rigorous simplified Bishop and generalised Janbu procedures, which predict, respectively, $F=0.9$ and $F=0.93$. For this case, PLAXIS 2D with strength reduction gives the lowest safety factor of $F=0 \cdot 86$. These results confirm that the finiteelement limit analysis method, incorporating pore pressures and strength reduction, gives believable slope stability predictions. Moreover, with the development of efficient adaptive meshing for this technique, tight upper and lower bounds on the safety factor can be found at low computational cost. Bearing in mind that a low factor of safety obtained by an approximate limit-equilibrium method is not necessarily correct, this feature is invaluable in practice.

\section{CONCLUSIONS}

New methods for performing geotechnical stability analysis in two and three dimensions have been described. The techniques are based on finite-element formulations of the limit theorems of classical plasticity, and incorporate an adaptive meshing strategy to give tight bounds on the collapse load. Unlike many limit-equilibrium methods, no assumptions regarding the shape of the failure surface need to be made in advance.

Finite-element limit analysis has several other important advantages that make it a very attractive option for geotechnical stability analysis. In no particular order of importance, these advantages include the following.

(a) The methods require only conventional strength parameters, such as $s_{\mathrm{u}}, c^{\prime}$ and $\phi^{\prime}$.

(b) The methods are ideally suited to strength reduction analysis, and hence they can provide a safety factor on strength as well as on load.

(c) The methods can model the effect of the pore pressures generated by steady-state seepage in a rigorous manner.

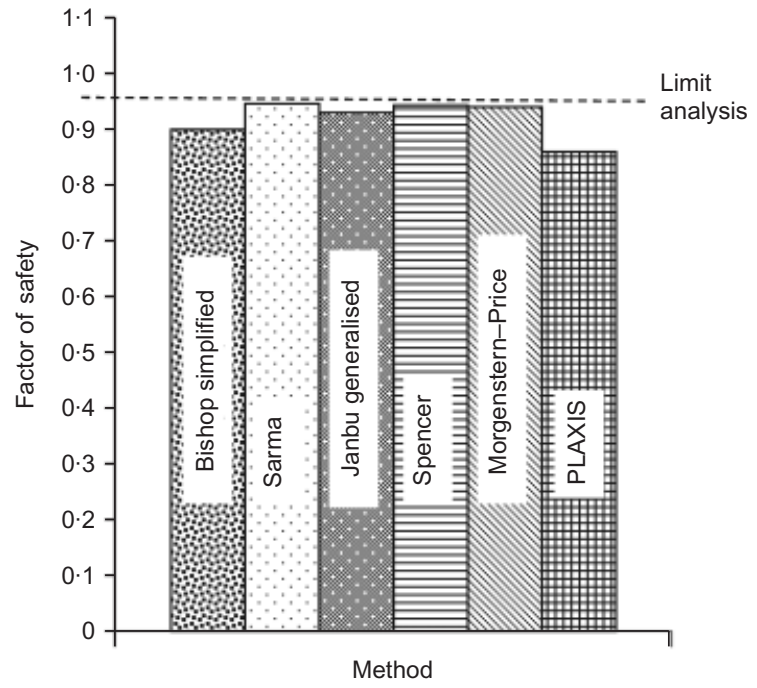

Fig. 64. Comparison of factors of safety for slope with weak layer and unconfined seepage flow

(d) The methods give the limit load directly, without the need to perform a complete incremental analysis. This is a major advantage in large-scale three-dimensional applications, where stability calculations using conventional displacement finite-element analysis are both difficult and time-consuming.

(e) The numerical solutions fulfil all the conditions of the limit theorems, so that the difference between the upper and lower bounds provides a direct estimate of the mesh discretisation error. This is an invaluable feature in practice, especially for cases where it is difficult to estimate the collapse load by other approximate techniques.

( $f$ ) The bounding property of the methods provides some insurance against operator error. Owing to the complexity of many geotechnical stability problems, this type of error can be difficult to detect with conventional approaches.

(g) The lower-bound solution can be used as the basis for design, with the upper-bound solution providing an accuracy check as well as an insight into the failure mechanism.

(h) Because they are founded on the finite-element concept, the methods can model heterogeneity, anisotropy, complex boundary shapes, complicated loading conditions and arbitrary geometries.

(i) Because the methods incorporate discontinuities in the stress and velocity fields, they are well suited to modelling jointed media and soil/structure interfaces.

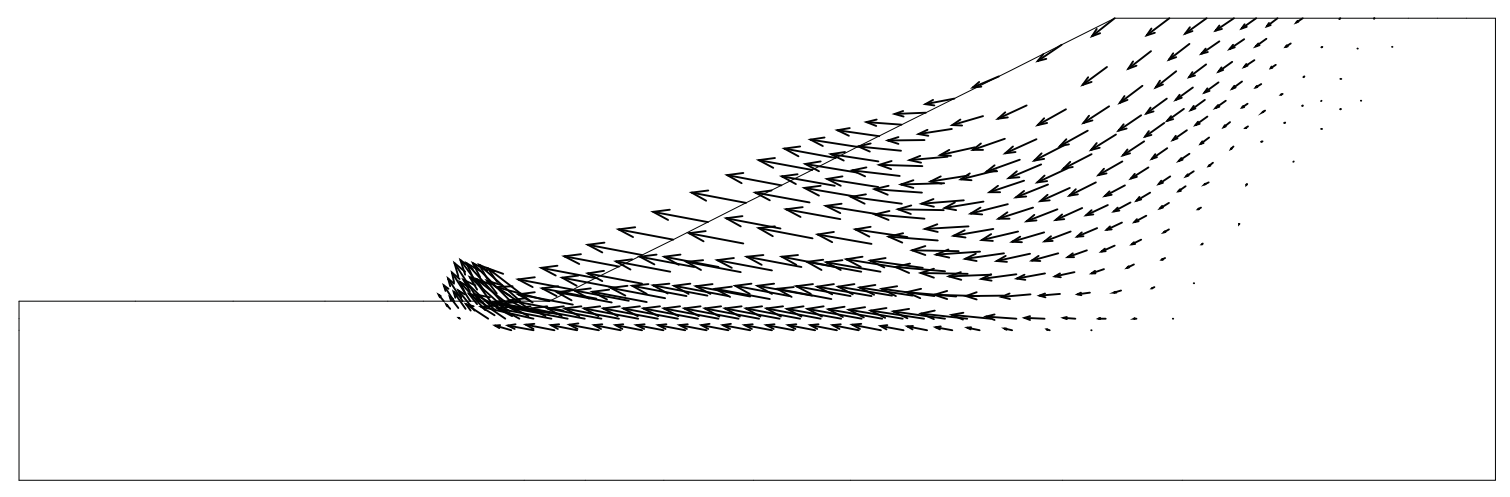

Fig. 63. Velocity vectors at collapse for slope with weak layer and unconfined seepage flow 
( $j$ ) Owing to recent advances in non-linear optimisation, the procedures are robust, efficient and straightforward to use.

(k) For materials with high friction angles, the effects of nonassociated flow can be modelled using the modified strength parameters proposed by Davis (1968).

\section{ACKNOWLEDGEMENTS}

The fundamental research reported in this lecture could not have taken place without the financial support of the Australian Research Council, which is currently funding the author's Australian Laureate Fellowship on 'Failure analysis of geotechnical infrastructure', as well as the ARC Centre of Excellence for Geotechnical Science and Engineering (headquartered at The University of Newcastle, Australia). Special thanks are also due to several industry partners, including Coffey Geotechnics, Douglas Partners and Advanced Geomechanics Pty Ltd, for their generous support of the Centre of Excellence.

During my career I have been especially fortunate to have benefited from the sage advice of several mentors, including the late John Booker, John Carter, Ian Donald, Harry Poulos and Mark Randolph. The geotechnical group at Newcastle has been a special place to work, and heartfelt thanks are due to Andrei Lyamin, Kristian Krabbenhøft, Richard Merifield, Daichao Sheng, Peter Kleeman, Andrew Abbo, Jim Hambleton and Majid Nazem. Internationally, Dave Potts and Lidija Zdravkovic at Imperial College were a great help in clarifying my ideas for the lecture, and Chris Martin from Oxford contributed important results. Other international collaborators on the work reported here include Charles Augarde (Durham), Antonio Gens (UPC Barcelona), Rodrigo Salgado (Purdue), Andrew Whittle (MIT) and Hai-Siu Yu (Nottingham).

Last, but not least, I should like to thank my wife Denise, my daughter Erica, and my sons Rory and Oscar for their patience and support during the writing of this lecture.

\section{NOTATION}

A matrix of constants

$A$ boundary area of soil mass; area of circular anchor

$A^{e}$ area of element $e$

$A_{q}$ boundary area of soil mass subjected to unknown surface tractions

$A_{t}$ boundary area of soil mass subjected to fixed surface tractions

$A_{w}$ boundary area of soil mass subjected to fixed velocities

$\overline{\mathbf{B}}$ global strain-displacement matrix for mesh multiplied by the element areas

$\mathbf{B}^{e}$ strain-displacement matrix for element $e$

$\overline{\mathbf{B}}^{e} \quad$ strain-displacement matrix for element $e$ multiplied by its area

$\mathbf{B}_{i} \quad$ strain-displacement matrix for node $i$ of an element

$\overline{\mathbf{B}}_{i} \quad$ strain-displacement matrix for node $i$ of an element multiplied by the element area

$B$ width of footing; width of biaxial sample

b vector of constants

$C$ tunnel cover

c vector of constants

$c$ cohesion

$c^{*}$ reduced cohesion parameter proposed by Davis

$c^{\prime}$ drained cohesion

$c_{\mathrm{a}}^{\prime}$ drained cohesion divided by factor of safety

$D$ tunnel diameter; diameter of circular anchor

$E_{i}$ target number of elements in current iteration of adaptive meshing process

$E_{\max }$ maximum number of elements allowed in adaptive meshing process

$e$ eccentricity of load applied to strip footing

$F$ bearing capacity factor for strip footing on clay with heterogeneous strength; factor of safety for slope based on shear strength
$\Delta F$ increment in factor of safety for slope

$f^{+}, f^{-}$positive and negative branches of planar MohrCoulomb yield criterion

$f\left(\sigma_{i j}\right), f(\boldsymbol{\sigma})$ yield surface

$\nabla f(\boldsymbol{\sigma})$ gradient of yield surface with respect to stresses

$G$ elastic shear modulus

$g$ vector of fixed body forces at a point

$\boldsymbol{g}^{e} \quad$ vector of fixed body forces for element $e$

$g$ plastic potential

$g_{x}, g_{y}$ fixed body forces in $x$ - and $y$-directions

$g_{x}^{e}, g_{y}^{e}$ fixed body forces in $x$ - and $y$-directions for element $e$

$\boldsymbol{H}$ global vector of unknown nodal heads

$\boldsymbol{H}^{e}$ vector of unknown nodal heads for element $e$

$\boldsymbol{H}_{0}$ global vector of fixed nodal heads

$H$ depth of circular anchor; depth of circular excavation; total head

$H^{i}$ total head at node $i$

$\boldsymbol{h}$ global vector of unknown body forces; vector of unknown body forces at a point

$\boldsymbol{h}^{e} \quad$ vector of unknown body forces for element $e$

$\boldsymbol{h}_{\mathrm{LB}}$ vector of lower-bound body forces

$\boldsymbol{h}_{\mathrm{UB}}$ vector of upper-bound body forces

$h_{x}, h_{y}$ unknown body forces in $x$ - and $y$-directions

$h_{x}^{e}, h_{y}^{e}$ unknown body forces in $x$ - and $y$-directions for element $e$

K flow matrix

$k$ soil permeability

$k_{i} \quad$ permeability at node $i$

$\bar{k}_{i} \quad$ smoothed permeability at node $i$

$L$ length of discontinuity; length of element edge; height of sample in biaxial test

$m$ gravity multiplier for slope equal to $\bar{\gamma} / \gamma$

$n, s$ local Cartesian coordinates in normal and tangential directions

$\mathbf{N}^{e}$ shape function matrix for element $e$

$N_{i}$ linear shape function for node $i$

$N_{\gamma} \quad$ breakout factor for circular anchor

$P$ eccentric load applied to strip footing; unsupported length of tunnel heading

$P_{\mathrm{H}}, P_{\mathrm{V}}$ horizontal and vertical components of eccentric load applied to strip footing

$P_{\mathrm{LB}}, P_{\mathrm{UB}} \quad$ lower and upper bounds on inclined eccentric load applied to strip footing

$p$ global vector of unknown nodal pore pressures

$p_{i} \quad$ pore pressure at node $i$

$\nabla p$ gradient of pore pressure field

$Q$ collapse load

$Q_{n}, Q_{s}$ normal and tangential (shear) loads per unit thickness acting on element edge of length $L$

$Q_{x}, Q_{y}$ element body force loads per unit thickness acting in $x$ - and $y$-directions

$Q_{\mathrm{u}} \quad$ load capacity of strip footing; load capacity of circular anchor

$q$ vector of unknown tractions acting on area $A_{q}$

$\boldsymbol{q}_{\mathrm{LB}}$ vector of lower-bound tractions acting on area $A_{q}$

$\boldsymbol{q}_{\mathrm{UB}}$ vector of upper-bound tractions acting on area $A_{q}$

$q$ bearing capacity

$q_{\mathrm{u}} \quad$ collapse pressure for biaxial test; pullout pressure for circular anchor

$q_{n}, q_{s}$ unknown normal and tangential (shear) stresses acting on element edge

$q_{n}^{i}, q_{s}^{i} \quad$ unknown normal and tangential (shear) stresses acting on element edge at node $i$

$R$ radius of circular failure surface about origin; radius of circular excavation

$R_{\mathrm{c}}$ radial distance to centre of footing from origin of circular failure surface

$s_{\mathrm{u}} \quad$ undrained shear strength

$s_{\mathrm{u} 0} \quad$ undrained shear strength at ground surface

$s(p)$ smoothed step function that lies between 0 and 1

$\boldsymbol{t}$ vector of fixed surface tractions acting on area $A_{t}$

$t_{n}^{i}, t_{s}^{i}$ fixed surface tractions in normal and tangential (shear) directions at node $i$

$\boldsymbol{u}$ global vector of unknown nodal velocities; vector of velocities at a point

$\boldsymbol{u}^{e}$ vector of unknown nodal velocities for element $e$ 
$\boldsymbol{u}^{i} \quad$ vector of unknown velocities at node $i$

$u_{n}, u_{s}$ unknown velocities in normal and tangential (shear) directions

$\Delta u_{n}, \Delta u_{s}$ velocity jumps across discontinuity in normal and tangential directions

$u_{n}^{i}, u_{s}^{i}$ unknown velocities in normal and tangential (shear) directions for node $i$

$\Delta u_{n}^{i j}, \Delta u_{s}^{i j} \quad$ velocity jumps across discontinuity in normal and tangential directions for nodal pair $(i, j)$

$u_{x}, u_{y}$ unknown velocities in $x$ - and $y$-directions

$u_{x}^{i}, u_{y}^{i}$ unknown velocities in $x$ - and $y$-directions for node $i$ volume of soil mass

$\dot{W}$ rate of internal energy dissipation less rate of work done by external loads

$W_{\text {ext }}, P_{\text {ext }}$ rate of work expended by external forces

$\dot{W}_{\text {int }}, P_{\text {int }}$ rate of internal energy dissipation

$w$ fixed velocities on surface $A_{w}$

$w_{n}, w_{s}$ fixed velocities in normal and tangential (shear) directions

$w_{n}^{i}, w_{s}^{i}$ fixed velocities in normal and tangential (shear) directions for node $i$

$x, y \quad$ Cartesian coordinates

$\boldsymbol{z}$ global vector of nodal elevation heads

$\dot{\alpha}$ plastic multiplier rate multiplied by element area

$\dot{\alpha}^{+}, \dot{\alpha}^{-} \quad$ plastic multiplier rate for positive and negative branches of planar Mohr-Coulomb yield criterion

$\dot{\alpha}^{+i j}, \dot{\alpha}^{-i j}$ plastic multiplier rate for positive and negative branches of planar Mohr-Coulomb yield criterion at nodal pair $(i, j)$

$\beta$ angle of normal to element edge; constant used in Davis' strength reduction formulae

$\Delta$ dissipation gap for mesh

$\Delta^{e} \quad$ dissipation gap for element $e$

$\gamma$ unit weight

$\bar{\gamma}$ mean of upper and lower bounds on maximum unit weight for slope

$\gamma_{\mathrm{LB}}, \gamma_{\mathrm{UB}}$ lower and upper bounds on unit weight

$\gamma_{\mathrm{w}}$ unit weight of water

$\delta$ width of velocity discontinuity

$\dot{\boldsymbol{\varepsilon}}^{\mathrm{p}} \quad$ vector of plastic strain rates

$\dot{\varepsilon}_{i j}^{\mathrm{p}} \quad$ plastic strain rates

$\dot{\varepsilon}_{\mathrm{v}}^{\mathrm{p}} \quad$ volumetric plastic strain rate

$\dot{\varepsilon}_{1}^{\mathrm{p}} \quad$ maximum principal plastic strain rate

$\theta$ angle subtended by circular failure surface

$\dot{\lambda}$ global vector of unknown plastic multiplier rates

$\dot{\lambda}$ plastic multiplier rate

$\lambda_{\mathrm{LB}}^{h}, \lambda_{\mathrm{UB}}^{h} \quad$ lower- and upper-bound load multipliers on body forces

$\lambda_{\mathrm{LB}}^{q}, \lambda_{\mathrm{UB}}^{q}$ lower- and upper-bound load multipliers on surface tractions

$v_{\mathrm{u}} \quad$ undrained Poisson's ratio

$\rho$ rate of undrained strength increase with depth

$\boldsymbol{\sigma}$ global vector of unknown nodal stresses; vector of stresses at a point

$\boldsymbol{\sigma}^{e} \quad$ vector of unknown stresses for element $e$

$\boldsymbol{\sigma}^{i} \quad$ vector of unknown stresses at node $i$

$\sigma_{\mathrm{LB}} \quad$ vector of lower-bound stresses

$\sigma_{\mathrm{UB}}$ vector of upper-bound stresses

$\boldsymbol{\sigma}_{\mathrm{LB}}^{e} \quad$ vector of lower-bound stresses for element $e$

$\boldsymbol{\sigma}_{\mathrm{UB}}^{e}$ vector of upper-bound stresses for element $e$

$\sigma_{i j} \quad$ stress tensor

$\sigma_{x x}, \sigma_{y y}, \tau_{x y} \quad$ Cartesian stresses

$\sigma_{x x}^{i}, \sigma_{y y}^{i}, \tau_{x y}^{i} \quad$ Cartesian stresses at node $i$

$\sigma_{n}$ normal stress

$\sigma_{n n}^{i} \quad$ normal stress at node $i$

$\sigma_{\mathrm{s}}$ ground surcharge

$\sigma_{\mathrm{t}}$ internal tunnel pressure

$\sigma_{1} \quad$ major principal stress

$\sigma_{3} \quad$ minor principal stress

$\tau$ shear stress

$\tau_{n s}^{i} \quad$ shear stress at node $i$

$\phi$ friction angle

$\phi^{*} \quad$ reduced friction angle proposed by Davis

$\phi^{\prime}$ drained friction angle

$\phi_{\mathrm{a}}^{\prime} \quad$ drained friction angle divided by factor of safety

$\phi_{\mathrm{u}}$ undrained friction angle $\psi^{\prime}$ dilation angle

$\dot{\omega}$ angular velocity of rigid rotating segment

\section{REFERENCES}

Abbo, A. J. \& Sloan, S. W. (1996). An automatic load stepping algorithm with error control. Int. J. Numer. Methods Engng 39, No. 10, 1737-1759.

Abbo, A. J. \& Sloan, S. W. (2000). SNAC (solid nonlinear analysis code) user manual, Version 2.0. Newcastle, Australia: University of Newcastle.

Almeida, R. C., Feijóo, R., Gleão, A. C., Padra, C. \& Silva, R. S. (2000). Adaptive finite element computational fluid dynamics using an anisotropic error estimator. Comput. Methods Appl. Mech. Engng 182, No. 3-4, 379-400.

Anderheggen, E. \& Knöpfel, H. (1972). Finite element limit analysis using linear programming. Int. J. Solids Struct. 8, No. 12, $1413-1431$.

Arai, K. \& Tagyo, K. (1985). Limit analysis of geotechnical problems by applying lower bound theorem. Soils Found. 25, No. 4, 37-48.

Assadi, A. \& Sloan, S. W. (1991). Undrained stability of a shallow square tunnel. J. Geotech. Div. ASCE 117, No. 8, 1152-1173.

Augarde, C. E., Lyamin, A. V. \& Sloan, S. W. (2003a). Prediction of undrained sinkhole collapse. J. Geotech. Geoenviron. Engng ASCE 129, No. 3, 197-205

Augarde, C. E., Lyamin, A. V. \& Sloan, S. W. (2003b). Stability of an undrained plane strain heading revisited. Comput. Geotech. 30, No. 5, 419-430.

Basudhar, P. K., Valsangkar, A. J. \& Madhav, M. R. (1979). Optimal lower bound of passive earth pressure using finite elements and nonlinear programming. Int. J. Numer. Analyt. Methods Geomech. 3, No. 4, 367-379.

Belytschko, T. \& Hodge, P. G (1970). Plane stress limit analysis by finite elements. J. Engng Mech. Div. ASCE 96, No. 6, 931944.

Biron, A. \& Charleux, G. (1972). Limit analysis of axisymmetric pressure vessel intersection of arbitrary shape. Int. J. Mech. Sci. 14, No. 1, 25-41.

Bishop, A. W. (1955). The use of slip circles in the stability analysis of earth slopes. Géotechnique 5, No. 1, 7-17, http:// dx.doi.org/10.1680/geot.1955.5.1.7.

Borges, L. A., Zouain, N., Costa, C. \& Feijóo, R. (2001). An adaptive approach to limit analysis. Int. J. Solids Struct. 38, No. 10-13, 1707-1720.

Bottero, A., Negre, R., Pastor, J. \& Turgeman, S. (1980). Finite element method and limit analysis theory for soil mechanics problems. Comput. Methods Appl. Mech. Engng 22, No. 1, $131-140$.

Britto, A. M. \& Kusakabe, O. (1982). Stability of unsupported axisymmetric excavations in soft clay. Géotechnique 32, No. 3, 261-270, http://dx.doi.org/10.1680/geot.1982.32.3.261.

Capsoni, A. \& Corradi, L. (1997). A finite element formulation of the rigid-plastic limit analysis problem. Int. J. Numer. Methods Engng 40, No. 11, 2063-2086.

Ciria, H. S. (2004). Computation of upper and lower bounds in limit state analysis using second-order cone programming and mesh adaptivity. $\mathrm{PhD}$ thesis, Department of Aeronautics and Astronautics, MIT, Cambridge, MA, USA.

Ciria, H., Peraire, J. \& Bonet, J. (2008). Mesh adaptive computation of upper and lower bounds in limit analysis. Int. J. Numer. Methods Engng 75, No. 8, 899-944.

Coulomb, C. A. (1773). Essai sur une application des règles de maximis \& minimis à quelques problèmes de statique, relatifs a l'architecture. Mém. Math. Phys. Acad. R. Sci. 7, 343-382. English translation: Note on an application of the rules of maximum and minimum to some statical problems, relevant to architecture. In Heyman, J. (1972) Coulomb's memoir on statics: An essay on the history of civil engineering. Cambridge, UK: Cambridge University Press.

Crank, J. (1984). Free and moving boundary problems. Oxford, UK: Clarendon Press.

Davis, E. H. (1968). Theories of plasticity and failure of soil masses. In Soil mechanics: selected topics (ed. I. K. Lee), pp. 341-354. New York, NY, USA: Elsevier. 
Davis, E. H. \& Booker, J. R. (1973). The effect of increasing strength with depth on the bearing capacity of clays. Géotechnique 23, No. 4, 551-563, http://dx.doi.org/10.1680/geot. 1973.23.4.551.

Davis, E. H., Gunn, M. J., Mair, R. J. \& Seneviratne, H. N. (1980) The stability of shallow tunnels and underground openings in cohesive material. Géotechnique 30, No. 4, 397-416, http:// dx.doi.org/10.1680/geot.1980.30.4.397.

Dawson, E. M., Roth, W. H. \& Drescher, A. (1999). Slope stability by strength reduction. Géotechnique 49, No. 6, 835-840, http:// dx.doi.org/10.1680/geot.1999.49.6.835.

De Borst, R. \& Vermeer, P. A. (1984). Possibilities and limitations of finite elements for collapse. Géotechnique 34, No. 2, 199210, http://dx.doi.org/10.1680/geot.1984.34.2.199.

Donald, I. B. \& Chen, Z. Y. (1997). Slope stability analysis by the upper bound approach: fundamentals and methods. Can. Geotech. J. 34, No. 6, 853-862.

Donald, I. B. \& Giam, S. K. (1989a). Soil slope stability programs review, ACADS publication No. U255. Melbourne, Australia: Association for Computer Aided Design.

Donald, I. B. \& Giam, S. K. (1989b). Improved comprehensive limit equilibrium stability analysis, Civil Engineering Research Report No. 1/1989. Melbourne, Australia: Monash University.

Drescher, A. \& Detournay, E. (1993). Limit load in translational mechanisms for associative and nonassociative materials. Géotechnique 43, No. 3, 443-456, http://dx.doi.org/10.1680/ geot.1993.43.3.443.

Drucker, D. C., Greenberg, W. \& Prager, W. (1951). The safety factor of an elastic plastic body in plane strain. Trans. ASME J. Appl. Mech. 73, 371-378.

Drucker, D. C., Prager, W. \& Greenberg, H. J. (1952). Extended limit design theorems for continuous media. Q. J. Appl. Math. 9, No. 4, 381-389

Duncan, J. M. \& Wright, S. G. (2005). Soil strength and slope stability. Hoboken, NJ, USA: John Wiley \& Sons.

Fletcher, R. \& Reeves, C. M. (1964). Function minimisation by conjugate gradient. Comput. J. 7, No. 2, 149-154.

Fortin, M. \& Glowinski, R. (1983). Augmented Lagrangian methods: Applications to the numerical solutions of boundary value problems, Studies in Mathematics and Its Applications, Vol. 15. Amsterdam, the Netherlands: North-Holland.

Friaâ, A. (1979). La loi de Norton-Hoff généralisée en plasticité et viscoplasticité. Thèse doctorat d'état, Université de Pierre et Marie Curie, Paris, France (in French).

GeoStudio (2007). Geo-Slope International, Calgary, Canada.

Giam, S. K. \& Donald, I. B. (1989a). Equivalence of limit equilibrium and upper-bound plasticity methods in soil stability analysis, Civil Engineering Research Report No. 4/1989. Melbourne, Australia: Monash University.

Giam, S. K. \& Donald, I. B. (1989b). Soil stability analysis by an upper-bound plasticity method. Civil Engineering Research Report No. 2/1989. Melbourne, Australia: Monash University.

Griffiths, D. V. (1982). Computation of bearing capacity factors using finite elements. Géotechnique 32, No. 3, 195-202, http:// dx.doi.org/10.1680/geot.1982.32.3.195.

Griffiths, D. V. \& Lane, P. A. (1999). Slope stability analysis by finite elements. Géotechnique 49, No. 3, 387-403, http:// dx.doi.org/10.1680/geot.1999.49.3.387.

Hjiaj, M., Lyamin, A. V. \& Sloan, S. W. (2004). Bearing capacity of a cohesive-frictional soil under non-eccentric inclined loading. Comput. Geotech. 31, No. 6, 491-516.

Hjiaj, M., Lyamin, A. V. \& Sloan, S. W. (2005). Numerical limit analysis solutions for the bearing capacity factor $N_{\gamma}$. Int. J. Solids Struct. 42, No. 5-6, 1681-1704.

Hodge, P. G. \& Belytschko, T. (1968). Numerical methods for the limit analysis of plates. J. Appl. Mech. 35, No. 4, 796-802.

Huh, H. \& Yang, W. H. (1991). A general algorithm for limit solutions of plane stress problems. Int. J. Solids Struct. 28, No. 6, 727-738.

Ilamparuthi, K., Dickin, E. A. \& Muthukrisnaiah, K. (2002). Experimental investigation of the uplift behaviour of circular plate anchors embedded in sand. Can. Geotech. J. 39, No. 3, 648-664.

Janbu, N. (1954). Application of composite slip surface for stability analysis. Proceedings of the European conference on stability of earth slopes, Stockholm, Vol. 3, pp. 43-49.
Janbu, N. (1973). Slope stability computations. In Embankment dam engineering: Casagrande volume (eds R. C. Hirschfield \& S. J. Poros), pp. 47-86. Hoboken, NJ, USA: Wiley.

Jiang, G. L. (1994). Regularised method in limit analysis. J. Engng Mech. Div. ASCE 120, No. 6, 1179-1197.

Jiang, G. L. (1995). Non-linear finite element formulation of kinematic limit analysis. Int. J. Numer. Methods Engng 38, No. 16, 2775-2807.

Kavlie, D. \& Moe, J. (1971). Automated design of frame structures. J. Struct. Div. ASCE 97, No. ST1, 33-61.

Kim, J., Salgado, R. \& Yu, H. S. (1999). Limit analysis of soil slopes subjected to pore water pressures. J. Geotech. Geoenviron. Engng. 125, No. 1, 49-58.

Krabbenhøft, K. \& Damkilde, L. (2003). A general nonlinear optimization algorithm for lower bound limit analysis. Int. J. Numer. Methods Engng 56, No. 2, 165-184.

Krabbenhøft, K., Lyamin, A. V., Hjiaj, M. \& Sloan, S. W. (2005). A new discontinuous upper bound limit analysis formulation. Int. J. Numer. Methods Engng 63, No. 7, 1069-1083.

Krabbenhøft, K., Lyamin, A.V. \& Sloan, S. W. (2007). Formulation and solution of some plasticity problems as conic programs. Int J. Solids Struct. 44, No. 5, 1533-1549.

Krabbenhøft, K., Lyamin, A. V. \& Sloan, S. W. (2008). Threedimensional Mohr-Coulomb limit analysis using semidefinite programming. Comm. Numer. Methods Engng 24, No. 11, $1107-1119$

Krabbenhøft, K., Karim, M. R., Lyamin, A. V. \& Sloan, S. W. (2012). Associated computational plasticity schemes for nonassociated frictional materials. Int. J. Numer. Methods Engng 90 , No. 9, 1089-1117.

Li, A. J., Merifield, R. S. \& Lyamin, A. V. (2008). Stability charts for rock slopes based on the Hoek-Brown failure criterion. Int. J. Rock Mech. Mining Sci. 45, No. 5, 689-700.

Li, A. J., Merifield, R. S. \& Lyamin, A. V. (2009a). Limit analysis solutions for three dimensional undrained slopes. Comput. Geotech. 36, No. 8, 1330-1351.

Li, A. J., Lyamin, A. V. \& Merifield, R. S. (2009b). Seismic rock slope stability charts based on limit analysis methods. Comput. Geotech. 36, No. 1-2, 135-148.

Li, A. J., Merifield, R. S. \& Lyamin, A. V. (2010). Three dimensional stability charts for slopes based on limit analysis methods. Can. Geotech. J. 47, No. 12, 1316-1334.

Liu, Y. H., Cen, Z. Z. \& Xu, B. Y. (1995). A numerical method for plastic limit analysis of 3-D structures. Int. J. Solids Struct. 32, No. 12, 1645-1658.

Lyamin, A. V. (1999). Three-dimensional lower bound limit analysis using nonlinear programming. $\mathrm{PhD}$ thesis, University of Newcastle, Australia.

Lyamin, A. V. \& Sloan, S. W. (2000). Stability of a plane strain circular tunnel in a cohesive-frictional soil. Proceedings of the $J$. $R$. Booker memorial symposium, Sydney, pp. 139-153.

Lyamin, A. V. \& Sloan, S. W. (2002a). Lower bound limit analysis using nonlinear programming. Int. J. Numer. Methods Engng 55, No. 5, 573-611.

Lyamin, A. V. \& Sloan, S. W. (2002b). Upper bound limit analysis using linear finite elements and nonlinear programming. Int. $J$. Numer. Analyt. Methods Geomech. 26, No. 2, 181-216.

Lyamin, A. V. \& Sloan, S. W. (2003). Mesh generation for lower bound limit analysis. Adv. Engng Software 34, No. 6, 321-338.

Lyamin, A. V., Krabbenhøft, K., Abbo, A. J. \& Sloan, S. W. (2005a). General approach for modelling discontinuities in limit analysis, Proc. 11th Int. Conf. Int. Assoc. Computer Methods Adv. Geomech., Torino 1, 95-102.

Lyamin, A. V., Sloan, S. W., Krabbenhøft, K. \& Hjiaj, M. (2005b). Lower bound limit analysis with adaptive remeshing. Int. J. Numer. Methods Engng 63, No. 14, 1961-1974.

Lysmer, J. (1970). Limit analysis of plane problems in soil mechanics. J. Soil Mech. Found. Div. ASCE 96, No. SM4, 13111334.

Maier, G., Zavelani-Rossi, A. \& Benedetti, D. (1972). A finite element approach to optimal design of plastic structures in plane stress. Int. J. Numer. Methods Engng 4, No. 4, 455-473.

Mair, R. J. (1979). Centrifugal modelling of tunnel construction in soft clay. $\mathrm{PhD}$ thesis, University of Cambridge, UK.

Makrodimopoulos, A. \& Martin, C. M. (2006). Lower bound limit analysis of cohesive-frictional materials using second-order cone 
programming. Int. J. Numer. Methods Engng 66, No. 4, 604634.

Makrodimopoulos, A. \& Martin, C. M. (2007). Upper bound limit analysis using simplex strain elements and second-order cone programming. Int. J. Numer. Analyt. Methods Geomech. 31, No. $6,835-865$.

Malkus, D. S. \& Hughes, T. J. R. (1978). Mixed finite element methods - reduced and selective integration techniques: a unification of concepts. Comput. Methods Appl. Mech. Engng 15, No. 1, 63-81.

Martin, C. M. (2004). User guide for ABC: Analysis of bearing capacity, Version 1.0. Oxford, UK: Department of Engineering Science, University of Oxford.

Merifield, R. S. \& Sloan, S. W. (2006). The ultimate pullout capacity of anchors in frictional soils. Can. Geotech. J. 43 No. 8, 852-868.

Merifield, R. S., Sloan, S. W. \& Yu, H. S. (1999). Rigorous solutions for the bearing capacity of two layered clay soils. Géotechnique 49, No. 4, 471-490, http://dx.doi.org/10.1680/ geot.1999.49.4.471.

Merifield, R. S., Sloan, S. W. \& Yu, H. S. (2001). Stability of plate anchors in undrained clay. Géotechnique 51, No. 2, 141-153, http://dx.doi.org/10.1680/geot.2001.51.2.141.

Merifield, R. S., Lyamin, A. V., Sloan, S. W. \& Yu, H. S. (2003). Three-dimensional stability analysis of plate anchors in clay. $J$. Geotech. Geoenviron. Engng ASCE 129, No. 3, 243-253.

Merifield, R. S., Lyamin, A. V. \& Sloan, S. W. (2005). The stability of inclined strip anchors in purely cohesive soil. J. Geotech. Geoenviron. Engng ASCE 131, No. 6, 792-799.

Merifield, R. S., Lyamin, A. V. \& Sloan, S. W. (2006a). Threedimensional lower bound solutions for the stability of plate anchors in sand. Géotechnique 56, No. 2, 123-132, http:// dx.doi.org/10.1680/geot.2006.56.2.123.

Merifield, R. S., Lyamin, A. V. \& Sloan, S. W. (2006b). Limit analysis solutions for the bearing capacity of rock masses using the generalised Hoek-Brown criterion. Int. J. Rock Mech. Mining Sci. 43, No. 6, 920-937.

Morgenstern, N. R. \& Price, V. E. (1965). The analysis of the stability of general slip surfaces. Géotechnique 15, No. 1, 79-93, http://dx.doi.org/10.1680/geot.1965.15.1.79.

Muñoz, J. J., Bonet, J., Huerta, A. \& Peraire, J. (2009). Upper and lower bounds in limit analysis: adaptive meshing strategies and discontinuous loading. Int. J. Numer. Methods Engng 77, No. 4, $471-501$.

Nagtegaal, J. C., Parks, D. M. \& Rice, J. R. (1974). On numerically accurate finite element solutions in the fully plastic range. Comput. Methods Appl. Mech. Engng 4, No. 2, 153-177.

Nelder, J. A. \& Mead, R. (1965). A simplex method for function minimisation. Comput. J. 7, No. 4, 308-315.

Nguyen, D. H., Trapletti, M. \& Ransart, D. (1978). Quasi-lower bounds and upper bounds of collapse loads of shells of revolution by the finite element method and by nonlinear programming. Int. J. Non-linear Mech. 13, No. 2, 79-102.

Palmer, A. C. (1966). A limit theorem for materials with nonassociated flow laws. J. Mécanique 5, No. 2, 217-222.

Pastor, J. (1978). Analyse limite: détermination numérique des solution statiques complètes: application au talus vertical. $J$. Mécanique Appl. 2, No. 2, 167-196 (in French).

Pastor, J. \& Turgeman, S. (1976). Mise en oeuvre numerique des methodes de l'analyse limite pour les materiaux de von Mises et de Coulomb standards en deformation plane. Mech. Res. Commun. 3, No. 6, 469-476 (in French).

Pastor, J. \& Turgeman, S. (1982). Limit analysis in axisymmetrical problems: numerical determination of complete statical solutions. Int. J. Mech. Sci. 24, No. 2, 95-117.

Pastor, M., Quecedo, M. \& Zienkiewicz, O. C. (1997). A mixed displacement-pressure formulation for numerical analysis of plastic failure. Comput. Struct. 62, No. 1, 13-23.

Pastor, M., Li, T., Liu, X. \& Zienkiewicz, O. C. (1999). Stabilized low-order finite elements for failure and localization problems in undrained soils and foundations. Comput. Methods Appl. Mech. Engng 174, No. 1-2, 219-234.

Pearce, A. (2000). Experimental investigation into the pullout capacity of plate anchors in sand. MSc thesis, University of Newcastle, Australia.

Peraire, J., Vahdati, M., Morgan, K. \& Zienkiewicz, O. C. (1987).
Adaptive remeshing for compressible flow computations. $J$. Comput. Phys. 72, No. 2, 449-466.

PLAXIS 2D (2011). Plaxis bv, Delft, the Netherlands.

Potts, D. M. (2003). Numerical analysis: a virtual dream or practical reality? Géotechnique 53, No. 6, 535-572, http://dx.doi.org/ 10.1680/geot.2003.53.6.535.

Potts, D. M. \& Gens, A. (1985). A critical assessment of methods of correcting for drift from the yield surface in elasto-plastic finite element analysis. Int. J. Numer. Analyt. Methods Geomech. 9, No. 2, 149-159.

Powell, M. J. D. (1964). An efficient method for finding the minimum of a function of several variables without calculating derivatives. Comput. J. 7, No. 2, 155-162.

Prandtl, L. (1920). Über die härte plastischer körper. Nachr. Ges. Wiss. Göttingen, Math.-Phys. Kl. 12, 74-85 (in German).

Raymond, G. P. (1967). The bearing capacity of large footings and embankments on clays. Géotechnique 17, No. 1, 1-10, http:// dx.doi.org/10.1680/geot.1967.17.1.1

Salgado, R., Lyamin, A. V., Sloan, S. W. \& Yu, H. S. (2004). Twoand three-dimensional bearing capacity of foundations in clay. Géotechnique 54, No. 5, 297-306, http://dx.doi.org/10.1680/ geot.2004.54.5.297.

Sarma, S. K. (1973). Stability analysis of embankments and slopes. Géotechnique 23, No. 3, 423-433, http://dx.doi.org/10.1680/ geot.1973.23.3.423.

Sarma, S. K. (1979). Stability analysis of embankments and slopes. J. Geotech. Engng Div. ASCE 105, No. 12, 1511-1524.

Sheng, D. C. \& Sloan, S. W. (2001). Load stepping schemes for critical state models. Int. J. Numer. Methods Engng 50, No. 1, $67-93$.

Shiau, S. H., Lyamin, A. V. \& Sloan, S. W. (2003). Bearing capacity of a sand layer on clay by finite element limit analysis. Can. Geotech. J. 40, No. 5, 900-915.

Sloan, S. W. (1979). Numerical prediction of collapse loads using finite element methods. MPhil thesis, University of Cambridge, UK.

Sloan, S. W. (1981). Numerical analysis of incompressible and plastic solids using finite elements. $\mathrm{PhD}$ thesis, University of Cambridge, UK.

Sloan, S. W. (1983). Discussion of 'Elastoplastic analyses of deep foundations in cohesive soil'. Int. J. Numer. Analyt. Methods Geomech. 7, No. 3, 385-393.

Sloan, S. W. (1987). Substepping schemes for the numerical integration of elastoplastic stress-strain relations. Int. J. Numer. Methods Engng 24, No. 5, 893-911.

Sloan, S. W. (1988a). Lower bound limit analysis using finite elements and linear programming. Int. J. Numer. Analyt. Methods Geomech. 12, No. 1, 61-67.

Sloan, S. W. (1988b). A steepest edge active set algorithm for solving sparse linear programming problems. Int. J. Numer. Methods Engng 26, No. 12, 2671-2685.

Sloan, S.W. (1989). Upper bound limit analysis using finite elements and linear programming. Int. J. Numer. Analyt. Methods Geomech. 13, No. 3, 263-282.

Sloan, S. W. \& Abbo, A. J. (1999). Biot consolidation analysis with automatic time stepping and error control. Part 2: Applications. Int. J. Numer. Analyt. Methods Geomech. 23, No. 6, 493-529.

Sloan, S. W. \& Assadi, A. (1991). Undrained stability of a square tunnel in a soil whose strength increases linearly with depth. Comput. Geotech. 12, No. 4, 321-346.

Sloan, S. W. \& Assadi, A. (1992). The stability of tunnels in soft ground. Proceedings of the Peter Wroth memorial symposium on predictive soil mechanics, Oxford, pp. 644-663.

Sloan, S. W. \& Assadi, A. (1994). Undrained stability of a plane strain heading. Can. Geotech. J., 31, No. 3, 443-450.

Sloan, S. W. \& Kleeman, P. W. (1995). Upper bound limit analysis with discontinuous velocity fields. Comput. Methods Appl. Mech. Engng 127, No. 1-4, 293-314.

Sloan, S. W. \& Randolph, M. F. (1982). Numerical prediction of collapse loads using finite element methods. Int. J. Numer. Analyt. Methods Geomech. 6, No. 1, 47-76.

Sloan, S. W. \& Randolph, M. F. (1983). Reply to discussion of 'Numerical prediction of collapse loads using finite element methods'. Int. J. Numer. Analyt. Methods Geomech. 7, No. 1, $135-141$

Sloan, S. W., Assadi, A. \& Purushothaman, N. (1990). Undrained 
stability of a trapdoor. Géotechnique 40, No. 1, 45-62, http:// dx.doi.org/10.1680/geot.1990.40.1.45.

Sloan, S. W., Abbo, A. J. \& Sheng, D. C. (2001). Refined explicit integration of elastoplastic models with automatic error control. Engng Comput. 18, No. 1/2, 121-154. Erratum (2002): Engng Comput. 19, No. 5/6, 594-594.

Small, J. C. (1977). Elasto-plastic consolidation of soils. $\mathrm{PhD}$ thesis, University of Sydney, Australia

Small, J. C., Booker, J. R. \& Davis, E. H. (1976). Elastoplastic consolidation of soil. Int. J. Solids Struct. 12, No. 6, 431-448.

Spencer, E. (1967). A method of analysis of the stability of embankments assuming parallel inter-slice forces. Géotechnique 17, No. 1, 11-26, http://dx.doi.org/10.1680/geot.1967.17.1.11.

Toh, C. T. \& Sloan, S. W. (1980). Finite element analysis of isotropic and anisotropic cohesive soils with a view to correctly predicting impending collapse. Int. J. Numer. Analyt. Methods Geomech. 4, No. 1, 1-23.

Turgeman, S. \& Pastor, J. (1982). Limit analysis: a linear formulation of the kinematic approach for axisymmetric mechanic problems. Int. J. Numer. Analyt. Methods Geomech. 6, No. 1, $109-128$.

Ukritchon, B., Whittle, A. J. \& Sloan, S. W. (1998). Undrained limit analysis for combined loading of strip footings on clay. $J$. Geotech. Geoenviron. Div. ASCE 124, No. 3, 265-276.

Ukritchon, B., Whittle, A. J. \& Sloan, S. W. (2003). Undrained stability of braced excavations in clay. J. Geotech. Geoenviron. Div. ASCE 129, No. 8, 738-755.

Wilson, D. W., Abbo, A. J., Sloan, S. W. \& Lyamin, A. V. (2011). Undrained stability of a circular tunnel where the shear strength increases linearly with depth. Can. Geotech. J. 48, No. 9, 13281342.

Yu, H. S., Sloan, S. W. \& Kleeman, P. W. (1994). A quadratic element for upper bound limit analysis. Engng Comput. 11, No. 3, 195-212.

Yu, H. S., Salgado, R., Sloan, S. W. \& Kim, J. M. (1998). Limit analysis versus limit equilibrium for slope stability. J. Geotech. Geoenviron. Engng ASCE 124, No. 1, 1-11.

Zienkiewicz, O. C. (1979). The finite element method. London, UK: McGraw-Hill.

Zienkiewicz, O. C., Humpheson, C. \& Lewis, R. W. (1975). Associated and non-associated visco-plasticity and plasticity in soil mechanics. Géotechnique 25, No. 4, 671-689, http:// dx.doi.org/10.1680/geot.1975.25.4.671.

Zouain, N., Herskovits, J., Borges, L. T. A. \& Feijóo, R. A. (1993). An iterative algorithm for limit analysis with nonlinear yield functions. Int. J. Solids Struct. 30, No. 10, 1397-1417.

\section{VOTE OF THANKS}

PROFESSOR H. G. POULOS, Coffey Geotechnics Pty Ltd, Australia.

When I first met Professor Sloan about 30 years ago, the late Professor Peter Wroth made a 'Class A' prediction that Scott Sloan would make an impact on the geotechnical world. Unlike some of our geotechnical predictions, Peter Wroth's was accurate, and over the following three decades Scott Sloan's career has blossomed. In particular, he has developed innovative applications of the finite-element method and applied these to a wide range of geotechnical stability problems. He has transformed a numerical method that had focused on load-deformation behaviour to one that can be applied to a range of problems that had previously defied confident analysis with the traditional finite-element, displacement-based approach. In addition, he has developed highly valuable parametric solutions, some of which he has presented this evening. Such solutions can be used both directly for routine geotechnical design and also for checking the results of more complex numerical techniques. The latter application is particularly important these days, when many analysts accept the results of their complex analyses without an adequately critical appraisal of their relevance and applicability to the problem in hand.

In this context, it is appropriate that we recall the following words of a former Rankine Lecturer, Professor David Potts: 'The potential of the numerical analysis in solving geotechnical problems is enormous. The potential for disaster is equally great if it is used by operators who do not understand soil mechanics principles and the concept of geotechnical design.' The work described by Professor Sloan this evening will assist in reducing the potential for disaster to which Professor Potts refers.

In recent years, Professor Sloan has built up a world-class research group at The University of Newcastle, a group that he leads with enthusiasm and aplomb, and in which the cooperative spirit that he embraces is strongly evident. The scope of research within this group has become quite broad, embracing not only traditional geotechnical engineering, but also materials technology and geoenvironmental and geochemical science. While much of the research is numerical, there is also, rightly, an emphasis on the verification of theoretical analyses via laboratory and field experiments. While his focus has been on research, Professor Sloan has also applied his techniques to practical problems involving considerable geological complexity - for example, the stability of retaining structures in stiff fissured clays existing in Botany Bay in Sydney Australia.

This Rankine Lecture has had something for everyone attending this evening

(a) intricate numerical details for the advanced analysts and software developers

(b) design charts and parametric solutions for the practitioner

(c) an increased understanding of failure mechanisms for all present.

We have had the privilege of listening to a person with a remarkable knowledge of numerical analysis and its application to geotechnical problems. It has been a stimulating and thought-provoking lecture, and members of our profession, both in academia and in practice, will eagerly await the culmination of his work through the software package to which he has referred. It is with great pleasure that I invite you all to show your appreciation to Professor Sloan for a most memorable Rankine Lecture. 